\title{
Perfluoroalkylation of Thiosulfonates: Synthesis of Perfluoroalkyl Sulfides
}

Ziwei Luo, Xinkan Yang and Gavin Chit Tsui*

Department of Chemistry, The Chinese University of Hong Kong, Shatin, New Territories, Hong Kong SAR

Table of Contents

General Experimental

General Experimental. Unless otherwise noted, reactions were carried out under argon in a $10 \mathrm{~mL}$ glass tube with magnetic stirring. Analytical thin layer chromatography (TLC) was performed with EM Science silica gel 60 F254 aluminum plates. Visualization was done under a UV lamp (254 nm) and by immersion in ethanolic phosphomolybdic acid (PMA) or potassium permanganate $\left(\mathrm{KMnO}_{4}\right)$, followed by heating using a heat gun. Organic solutions were concentrated by rotary evaporation at $23-40{ }^{\circ} \mathrm{C}$. Purification of reaction products were generally done by flash column chromatography with Grace Materials Technologies 230-400 mesh silica gel.

Materials. (Trifluoromethyl)trimethylsilane was purchased from Sigma-Aldrich. (Pentafluoroethyl)trimethylsilane and (heptafluoropropyl)trimethylsilane was purchased from TCI. Anhydrous DMSO, DMF and sodium acetate was purchased from J\&K Scientific. Sodium hydrogen carbonate (extra pure) was purchased from Riedel-de Haen. Fluoroform (Research Grade, Purity: $99.999 \%$ min., 9.1kg in 16 L size cylinder) was purchased from SynQuest Laboratories, USA. Halocarbon 125-Pentafluoroethane (Purity: 99.0\% min., $9.1 \mathrm{~kg}$ in $16 \mathrm{~L}$ size cylinder) was purchased from SCIENTIFIC GAS ENGINEERING CO., LTD. Potassium tert-butoxide (97\%) was purchased from Alfa Aesar. Other chemicals for substrates preparation were purchased from Acros, J\&K Scientific, Aldrich, Dikemann, Bide and Heowns.

Instrumentation. Proton nuclear magnetic resonance spectra $\left({ }^{1} \mathrm{H}\right.$ NMR) spectra, carbon nuclear magnetic resonance spectra $\left({ }^{13} \mathrm{C} \mathrm{NMR}\right)$ and fluorine nuclear magnetic resonance spectra $\left({ }^{19} \mathrm{~F} \mathrm{NMR}\right)$ were recorded at 23 ${ }^{\circ} \mathrm{C}$ on a Bruker 400 spectrometer in $\mathrm{CDCl}_{3}\left(400 \mathrm{MHz}\right.$ for ${ }^{1} \mathrm{H}, 101 \mathrm{MHz}$ for ${ }^{13} \mathrm{C}$ and $377 \mathrm{MHz}$ for ${ }^{19} \mathrm{~F}$ ) and Bruker 500 spectrometer in $\mathrm{CDCl}_{3}\left(500 \mathrm{MHz}\right.$ for ${ }^{1} \mathrm{H}, 126 \mathrm{MHz}$ for ${ }^{13} \mathrm{C}$ and $471 \mathrm{MHz}$ for $\left.{ }^{19} \mathrm{~F}\right)$. Chemical shifts for protons were reported as parts per million in $\delta$ scale using solvent residual peak $\left(\mathrm{CHCl}_{3}: 7.26 \mathrm{ppm}\right)$ or tetramethylsilane $(0.00 \mathrm{ppm})$ as internal standards. Chemical shifts of ${ }^{13} \mathrm{C}$ NMR spectra were reported in ppm from the central peak of $\mathrm{CDCl}_{3}(77.16 \mathrm{ppm})$ on the $\delta$ scale. Chemical shifts of ${ }^{19} \mathrm{~F} \mathrm{NMR}$ are reported as parts per million in $\delta$ scale using benzotrifluoride $(-63.72 \mathrm{ppm})$ as internal standards. Data are represented as follows: chemical shift, integration, multiplicity $(\mathrm{s}=$ singlet, $\mathrm{d}=$ doublet, $\mathrm{t}=$ triplet, $\mathrm{q}=$ quartet, $\mathrm{qn}=$ quintuplet, $\mathrm{sx}=$ sextet, $\mathrm{sp}=$ septuplet, $\mathrm{m}=$ multiplet, $\mathrm{br}=$ broad $)$, and coupling constant $(J, \mathrm{~Hz})$. High resolution mass spectra (HRMS) were obtained on a Thermo Q Exactive Focus Orbitrap Mass Spectrometer or a Bruker 9.4T ICR Mass Spectrometer. 


\section{Experimental Procedures.}

\section{General procedure A: perfluoroalkylation of thiosulfonates using $\mathrm{TMS}\left(\mathrm{CF}_{2}\right)_{\mathrm{n}} \mathrm{CF}_{3}$ :}

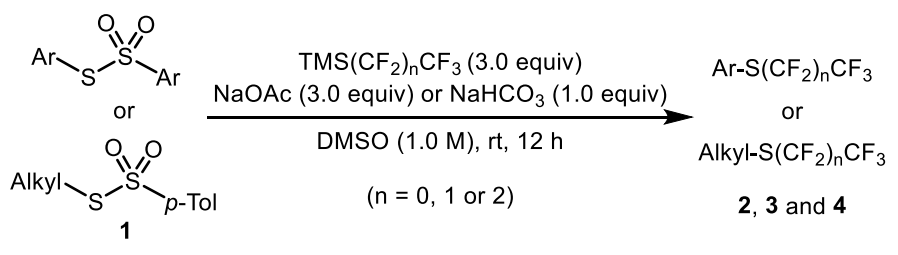

To a glass tube charged with a magnetic stir bar was added thiosulfonate $\mathbf{1}(0.2 \mathrm{mmol})$ and NaOAc (49.2 $\mathrm{mg}$, $0.6 \mathrm{mmol}$, for $\mathbf{2 a - y}, \mathbf{2 a d}, \mathbf{3 a - b}$ and $\mathbf{4 a - b})$ or $\mathrm{NaHCO}_{3}(16.8 \mathrm{mg}, 0.2 \mathrm{mmol}$, for $\mathbf{2 z}, \mathbf{2} \mathbf{a a}$, 2ab and $\mathbf{2 a c})$. The tube was then evacuated and flushed with argon. Anhydrous DMSO $(0.2 \mathrm{~mL})$ was then added followed by dropwise addition of $\mathrm{TMS}\left(\mathrm{CF}_{2}\right)_{\mathrm{n}} \mathrm{CF}_{3}(\mathrm{n}=0,1$ or 2$)(0.6 \mathrm{mmol})$ with stirring. The reaction mixture was stirred at room temperature for $12 \mathrm{~h}$ under argon, then diluted with DCM. The organic layer was washed with water (three times) and brine, then dried over anhydrous $\mathrm{Na}_{2} \mathrm{SO}_{4}$. After filtration, the filtrate was concentrated under reduced pressure. The residue was purified by flash chromatography on silica gel to afford the corresponding perfluoroalkyl sulfides $(\mathbf{2}, \mathbf{3}$ and $\mathbf{4})$.

\section{Preparation of perfluoroalkyl sulfides using thiosulfonates and $\mathrm{H}\left(\mathrm{CF}_{2}\right)_{\mathrm{n}} \mathrm{CF}_{3}$ :}

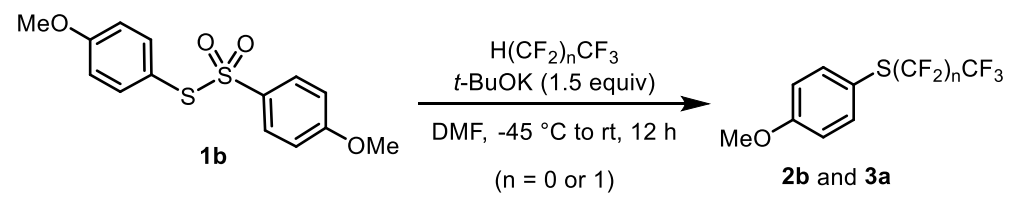

In a glove box, to a glass tube charged with a magnetic stir bar was added $t$-BuOK (33.6 mg, $0.3 \mathrm{mmol})$. The tube was sealed with a septum, brought out of the glove box, then $\mathbf{1 b}(62.1 \mathrm{mg}, 0.2 \mathrm{mmol})$ was quickly added and the tube was sealed again. After being evacuated and flushed with argon, the tube was moved to $-45^{\circ} \mathrm{C}$ for 5 min. Pre-cooled anhydrous DMF $\left(-45^{\circ} \mathrm{C}, 1.0 \mathrm{~mL}\right)$ was then added to the mixture. After being evacuated again, the tube was refilled with $\mathrm{H}\left(\mathrm{CF}_{2}\right)_{n} \mathrm{CF}_{3}(\mathrm{n}=0$ or 1$)$ by a balloon through a needle. The reaction mixture was then gradually warmed up to room temperature. After being stirred for $12 \mathrm{~h}$, the reaction mixture was diluted with DCM. The organic layer was washed with water (three times) and brine, then dried over anhydrous $\mathrm{Na}_{2} \mathrm{SO}_{4}$. After filtration, the filtrate was concentrated under reduced pressure. The residue was purified by flash chromatography on silica gel to afford 4-methoxyphenyl trifluoromethyl sulfides $\mathbf{2 b}(0.11 \mathrm{mmol}, 23.2 \mathrm{mg}, 56$ $\%)$ or 4-methoxyphenyl pentafluoroethyl sulfides $\mathbf{3 a}(0.13 \mathrm{mmol}, 33.5 \mathrm{mg}, 65 \%)$.

\section{1 mmol scale reaction:}
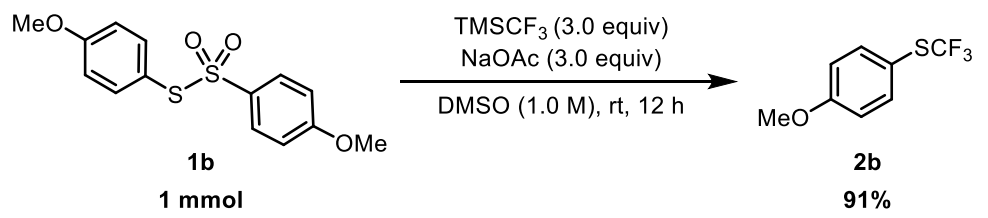

To a glass tube charged with a magnetic stir bar was added $\mathbf{1 b}(310.4 \mathrm{mg}, 1.0 \mathrm{mmol})$ and NaOAc $(246.0 \mathrm{mg}$, $3.0 \mathrm{mmol})$. The tube was then evacuated and flushed with argon. Anhydrous DMSO (1.0 mL) was then added followed by dropwise addition of $\mathrm{TMSCF}_{3}(0.45 \mathrm{~mL}, 3.0 \mathrm{mmol})$ with stirring. The reaction mixture was stirred at room temperature for $12 \mathrm{~h}$ under argon, then diluted with DCM. The organic layer was washed with water (three times) and brine, then dried over anhydrous $\mathrm{Na}_{2} \mathrm{SO}_{4}$. After filtration, the filtrate was concentrated under reduced pressure. The residue was purified by flash chromatography on silica gel to afford the corresponding trifluoromethyl sulfide $\mathbf{2 b}$ (0.91 mmol, $188.9 \mathrm{mg}, 91 \%)$. 


\section{Substrates:}

\section{Aryl substrates (1a-l):}

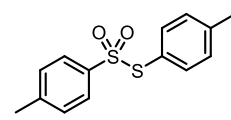<smiles>Cc1ccc(S(=O)(=O)c2ccc(C)cc2)cc1</smiles>

1b

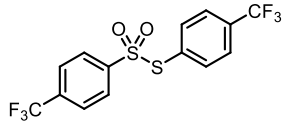

1c

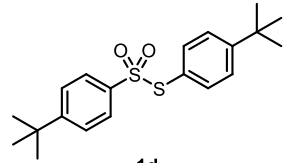

1d<smiles>N#Cc1ccc(SS(=O)(=O)c2ccc(C#N)cc2)cc1</smiles>

$1 \mathrm{e}$<smiles>CC(=O)c1ccc(SS(=O)(=O)c2ccc(C(C)=O)cc2)cc1</smiles>

$1 f$<smiles>O=S(=O)(Sc1ccc(Br)cc1)c1ccc(Br)cc1</smiles>

$1 \mathrm{~h}$<smiles>O=S(=O)(Sc1cccc(Br)c1)c1cccc(Br)c1</smiles>

$1 \mathrm{i}$<smiles>O=S(=O)(Sc1cccs1)c1cccs1</smiles>

$1 \mathrm{j}$<smiles></smiles>

$1 \mathrm{k}$<smiles>O=S(=O)(Sc1ccc2c(c1)CCO2)c1ccc2c(c1)CCO2</smiles>

11

Alkyl substrates (1m-ad):<smiles>Cc1ccc(S(=O)(=O)CCCCCOC(=O)c2ccccc2)cc1</smiles>

$1 \mathrm{~m}$<smiles>Cc1ccc(S(=O)(=O)SCCOC(=O)c2ccccc2)cc1</smiles>

1n<smiles>Cc1ccc(S(=O)(=O)CCCCCOC(=O)CCc2ccccc2)cc1</smiles>

10<smiles>Cc1ccc(S(=O)(=O)SCC2CCN(C(=O)c3ccccc3)CC2)cc1</smiles>

$1 \mathrm{p}$<smiles>Cc1ccc(S(=O)(=O)CCCCCN2C(=O)c3ccccc3C2=O)cc1</smiles>

19<smiles>CCC(SC(C)(O)c1ccc(C)cc1)c1ccccc1</smiles>

1u<smiles>Cc1ccc(S(=O)(=O)Cc2ccc(Br)cc2)cc1</smiles>

1y<smiles>Cc1ccc(S(=O)(=O)CCCCCO[Si](c2ccccc2)(c2ccccc2)C(C)(C)C)cc1</smiles><smiles>Cc1ccc(S(=O)(=O)SCCCc2ccccc2)cc1</smiles><smiles>Cc1ccc(S(=O)(=O)CCc2ccccc2)cc1</smiles><smiles>COc1ccc(CSS(=O)(=O)c2ccc(C)cc2)cc1</smiles>

1v<smiles>Cc1ccc(S(=O)(=O)SCc2ccc(C(C)(C)C)cc2)cc1</smiles>

1w<smiles>Cc1ccc(S(=O)(=O)SCc2ccc(Cl)cc2)cc1</smiles>

$1 x$<smiles>Cc1ccc(S(=O)(=O)SCC#Cc2ccccc2)cc1</smiles><smiles>Cc1ccc(S(=O)(=O)OCC(CSS(=O)(=O)c2ccccc2)NC(=O)c2ccccc2)cc1</smiles>

1 ac 1aa<smiles>Cc1ccc(S(=O)(=O)SC/C=C/c2ccccc2)cc1</smiles><smiles>C#CC1(O)CCC2[C@H]3Cc4cc(OCCCCCSS(=O)(=O)c5ccc(C)cc5)ccc4C3CCC21C</smiles>

The aryl substrates 1a-l were synthesized according to the literature procedures ${ }^{1-2}$ from the corresponding aryl sulfonyl chloride. The alkyl substrates $\mathbf{1 m - a d}$ were synthesized according to the literature procedures ${ }^{3-4}$ from the corresponding alkyl bromide or $N$-Boc- $L$-cysteinate. 
General procedure B: synthesis of aryl substrates (1a-b, 1d-l): ${ }^{1}$

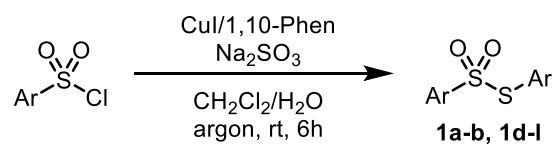

To a round-bottom flask charged with a magnetic stir bar was added aryl sulfonyl chloride (1.0 equiv.), $\mathrm{Na}_{2} \mathrm{SO}_{3}$ (3.0 equiv.), $\mathrm{CuI}(2.5 \mathrm{~mol} \%)$ and 1,10-Phen (2.5 mol\%). The flask was then evacuated and flushed with argon. Degassed DCM $(0.2 \mathrm{M})$ and $\mathrm{H}_{2} \mathrm{O}$ (1.0 equiv.) was then added. The resulting mixture was stirred at room temperature overnight. After being diluted with DCM, the mixture was washed with water (three times) and brine, then dried over anhydrous $\mathrm{Na}_{2} \mathrm{SO}_{4}$. After filtration, the filtrate was concentrated by rotary evaporator under reduced pressure. The residue was purified by flash chromatography on silica gel to afford 1a-b and 1d-l.

Preparation of $S$-4-(trifluoromethyl)phenyl-4-trifluoromethylbenzenesulfono-thioate (1c): ${ }^{2}$

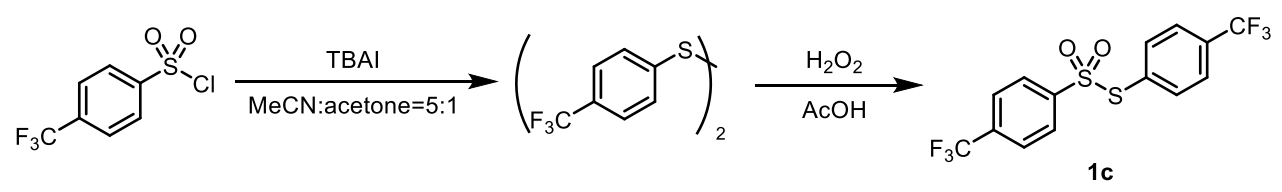

To a solution of 4-trifluoromethylbenzenesulfonyl chloride (978.5 mg, $4.0 \mathrm{mmol})$ in MeCN/acetone (10.0/2.0 $\mathrm{mL})$ in a round-bottom flask charged with a magnetic stir bar, a solution of $n$ - $\mathrm{Bu}_{4} \mathrm{NI}(3.8 \mathrm{~g}, 12 \mathrm{mmol})$ in $\mathrm{MeCN} /$ acetone $(10.0 / 2.0 \mathrm{~mL})$ was slowly added by a syringe. After stirring at room temperature for 20 hours, the solvents were evaporated. The residue was purified by flash chromatography on silica gel to afford 1,2bis(4-(trifluoromethyl)phenyl)disulfane.

To a solution of 1,2-bis(4-(trifluoromethyl)phenyl)disulfane $(288.8 \mathrm{mg}, 0.8 \mathrm{mmol})$ dissolved in acetic acid $(2.0 \mathrm{~mL})$ in a round-bottom flask charged with a magnetic stir bar was added $30 \%$ aq. $\mathrm{H}_{2} \mathrm{O}_{2}(0.16 \mathrm{~mL}, 1.6$ $\mathrm{mmol}$ ) dropwise at $0{ }^{\circ} \mathrm{C}$. After gradually warming to room temperature, the mixture was stirred for $48 \mathrm{~h}$, then water $(2.0 \mathrm{~mL})$ was added. The mixture was extracted with DCM (three times). The combined organic extract was washed with saturated $\mathrm{NaHCO}_{3}$, then dried over anhydrous $\mathrm{Na}_{2} \mathrm{SO}_{4}$. After filtration, the filtrate was concentrated under reduced pressure. The residue was purified by flash chromatography on silica gel to afford $S$-(4-(trifluoromethyl)phenyl) 4-(trifluoromethyl)benzenesulfonothioate (1c) as a white solid (0.58 mmol, 223.2 $\mathrm{mg}, 72 \%), \mathrm{R}_{\mathrm{f}}=0.3$ (hexane/EA = 10:1). ${ }^{1} \mathbf{H}$ NMR $\left(500 \mathrm{MHz}, \mathrm{CDCl}_{3}\right): \delta 7.75-7.71(\mathrm{~m}, 4 \mathrm{H}), 7.65(\mathrm{~d}, J=8.2$ $\mathrm{Hz}, 2 \mathrm{H}), 7.54(\mathrm{~d}, J=8.1 \mathrm{~Hz}, 2 \mathrm{H}) \mathrm{ppm} .{ }^{13} \mathrm{C}$ NMR $\left(126 \mathrm{MHz}, \mathrm{CDCl}_{3}\right): \delta 146.22,136.83,135.66\left(\mathrm{q}, J_{C-F}=33.2\right.$ $\mathrm{Hz}), 133.76\left(\mathrm{q}, J_{C-F}=33.1 \mathrm{~Hz}\right), 131.71,128.06,126.63\left(\mathrm{q}, J_{C-F}=3.6 \mathrm{~Hz}\right), 126.43\left(\mathrm{q}, J_{C-F}=3.6 \mathrm{~Hz}\right), 123.45(\mathrm{~d}$, $\left.J_{C-F}=272.9 \mathrm{~Hz}\right), 123.02\left(\mathrm{~d}, J_{C-F}=273.3 \mathrm{~Hz}\right) \mathrm{ppm} .{ }^{19} \mathbf{F}$ NMR $\left(471 \mathrm{MHz}, \mathrm{CDCl}_{3}\right): \delta-64.15(\mathrm{~s}, 3 \mathrm{~F}),-64.26(\mathrm{~s}, 3 \mathrm{~F})$ $\mathrm{ppm}$. The spectral data are in full accordance with the literature report. ${ }^{2 \mathrm{a}}$

General procedure C: synthesis of alkyl substrates $(1 \mathrm{~m}-\mathrm{ab}, 1 \mathrm{ad}):^{3}$

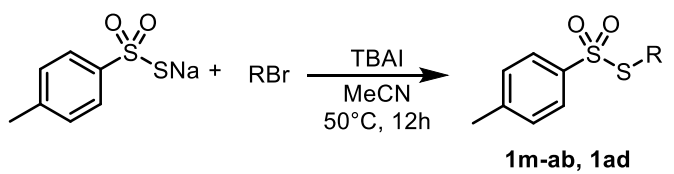

To a round-bottom flask charged with a magnetic stir bar was added alkyl bromide (1.2 equiv.), $p$-TolSO $\mathrm{SNa}_{2}$ (1.0 or 1.2 equiv.) and TBAI $(5 \mathrm{~mol} \%)$. $\mathrm{MeCN}(0.25 \mathrm{M})$ was then added to give a suspension. After the flask was sealed with a septum, the mixture was placed into a $50{ }^{\circ} \mathrm{C}$ oil bath and stirred for $12 \mathrm{~h}$. Then the reaction was cooled to room temperature. The mixture was filtered and the residue was washed by DCM (three times). The combined filtrate was concentrated under reduced pressure, then the residue was purified by flash chromatography on silica gel to afford $\mathbf{1 m - a b}$ and $\mathbf{1 a d}$. 


\section{Preparation of ethyl $N$-(tert-butoxycarbonyl)-S-tosyl- $L$-cysteinate (1ac) $:^{4}$}

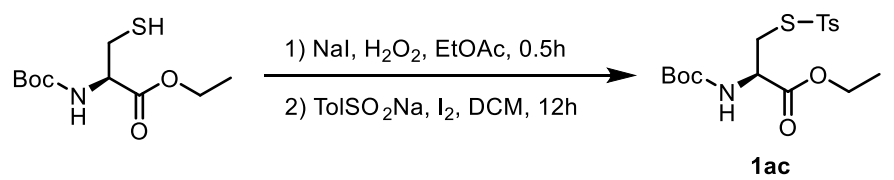

To a solution of ethyl $N$-(tert-butoxycarbonyl)- $L$-cysteinate $(997.3 \mathrm{mg}, 4.0 \mathrm{mmol})$ in EtOAc $(12 \mathrm{~mL})$ in a round-bottom flask charged with a magnetic stir bar was added $\mathrm{NaI}(6.0 \mathrm{mg}, 0.04 \mathrm{mmol})$ and $30 \%$ aq. $\mathrm{H}_{2} \mathrm{O}_{2}$ $(0.44 \mathrm{~mL}, 4.0 \mathrm{mmol})$ and the mixture was stirred at room temperature for $0.5 \mathrm{~h}$. The solvent was removed under reduced pressure and the residue was used as disulfide directly without purification.

To a mixture of sodium $p$-toluenesulfonate $(1.14 \mathrm{~g}, 6.4 \mathrm{mmol})$ and crude disulfide in DCM $(10 \mathrm{~mL})$ in a round-bottom flask was added $\mathrm{I}_{2}(1.02 \mathrm{~g}, 4.0 \mathrm{mmol})$ with mixing. The mixture was stirred for $12 \mathrm{~h}$, then diluted with DCM and washed with saturated $\mathrm{Na}_{2} \mathrm{~S}_{2} \mathrm{O}_{3}$ and water. The organic layer was then dried over anhydrous $\mathrm{Na}_{2} \mathrm{SO}_{4}$. After filtration, the filtrate was concentrated under reduced pressure. The residue was purified by flash chromatography on silica to afford $N$-(tert-butoxycarbonyl)- $S$-tosyl- $L$-cysteinate (1ac) as a white solid (3.57 mmol, $1.44 \mathrm{~g}, 89 \%), \mathrm{R}_{\mathrm{f}}=0.2$ (hexane/EA = 4:1). ${ }^{1} \mathbf{H}$ NMR (400 MHz, CDCl $): \delta 7.67(\mathrm{~d}, J=8.3 \mathrm{~Hz}, 2 \mathrm{H}), 7.22$ $(\mathrm{d}, J=8.3 \mathrm{~Hz}, 2 \mathrm{H}), 5.51(\mathrm{~d}, J=7.6 \mathrm{~Hz}, 1 \mathrm{H}), 4.38(\mathrm{~d}, J=5.6 \mathrm{~Hz}, 1 \mathrm{H}), 4.04(\mathrm{q}, J=7.1 \mathrm{~Hz}, 2 \mathrm{H}), 3.33(\mathrm{ddd}, J=$ $20.2 \mathrm{~Hz}, J=13.9 \mathrm{~Hz}, J=5.5 \mathrm{~Hz}, 2 \mathrm{H}), 2.31(\mathrm{~s}, 3 \mathrm{H}), 1.30(\mathrm{~s}, 9 \mathrm{H}), 1.12(\mathrm{t}, J=7.2 \mathrm{~Hz}, 3 \mathrm{H}) \mathrm{ppm} .{ }^{13} \mathbf{C} \mathbf{~ N M R}(101$ $\left.\mathrm{MHz}, \mathrm{CDCl}_{3}\right): \delta 169.48,154.77,144.84,141.23,129.71,126.76,79.85,61.75,52.63,37.22,27.90,21.32,13.75$ $\mathrm{ppm}$. The spectral data are in full accordance with the literature report. ${ }^{5}$ 
Table S1. Optimization studies. ${ }^{\mathrm{a}}$

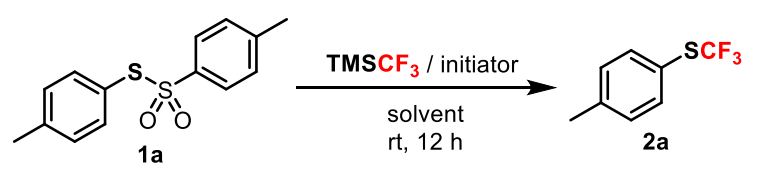

\begin{tabular}{|c|c|c|c|c|}
\hline entry & $\begin{array}{l}\text { equiv of } \\
\mathrm{TMSCF}_{3}\end{array}$ & initiator (equiv) & solvent $(\mathrm{M})$ & $\mathbf{2 a}(\%)^{\mathrm{b}}$ \\
\hline 1 & 1.5 & $\mathrm{NaOAc}(1.5)$ & DMSO (0.2) & 39 \\
\hline 2 & 2.0 & $\mathrm{NaOAc}(2.0)$ & DMSO (0.2) & 52 \\
\hline 3 & 3.0 & $\mathrm{NaOAc}(3.0)$ & DMSO (0.2) & 77 \\
\hline 4 & 3.0 & $\mathrm{NaOAc}(3.0)$ & DMSO (0.1) & 42 \\
\hline 5 & 3.0 & $\mathrm{NaOAc}(3.0)$ & DMSO (0.5) & 88 \\
\hline 6 & 3.0 & NaOAc (3.0) & DMSO (1.0) & $87(47)^{c, d}$ \\
\hline $7^{\mathrm{e}}$ & 3.0 & $\mathrm{NaOAc}(3.0)$ & DMSO (1.0) & 68 \\
\hline $8^{f}$ & 3.0 & NaOAc (3.0) & DMSO (1.0) & $94(75)^{c, d}$ \\
\hline 9 & 2.0 & $\mathrm{NaOAc}(3.0)$ & DMSO (1.0) & 68 \\
\hline 10 & 3.0 & $\mathrm{NaOAc}(2.0)$ & DMSO (1.0) & 83 \\
\hline 11 & 3.0 & $\mathrm{NaOAc}(1.0)$ & DMSO (1.0) & 82 \\
\hline 12 & 3.0 & $\mathrm{NaOAc}(0.2)$ & DMSO (1.0) & 63 \\
\hline 13 & 3.0 & $\mathrm{NaOAc}(3.0)$ & DMF (1.0) & 86 \\
\hline 14 & 3.0 & $\mathrm{NaOAc}(3.0)$ & $\operatorname{MeCN}(1.0)$ & 4 \\
\hline 15 & 3.0 & $\mathrm{NaOAc}(3.0)$ & 1,4-dioxane (1.0) & 0 \\
\hline 16 & 3.0 & $\mathrm{NaOAc}(3.0)$ & THF (1.0) & 0 \\
\hline 17 & 3.0 & $\mathrm{NaOAc}(3.0)$ & toluene (1.0) & 0 \\
\hline 18 & 3.0 & KOAc (3.0) & DMSO (1.0) & 85 \\
\hline 19 & 3.0 & LiOAc (3.0) & DMSO (1.0) & 87 \\
\hline 20 & 3.0 & $\mathrm{KF}(3.0)$ & DMSO (1.0) & 79 \\
\hline 21 & 3.0 & TBAF· $3 \mathrm{H}_{2} \mathrm{O}(3.0)$ & DMSO (1.0) & 3 \\
\hline 22 & 3.0 & $\mathrm{~K}_{2} \mathrm{CO}_{3}(3.0)$ & DMSO (1.0) & 71 \\
\hline 23 & 3.0 & $\mathrm{NaHCO}_{3}(3.0)$ & DMSO (1.0) & 73 \\
\hline 24 & 3.0 & $\mathrm{Et}_{3} \mathrm{~N}(3.0)$ & DMSO (1.0) & 69 \\
\hline 25 & 3.0 & none & DMSO (1.0) & 8 \\
\hline
\end{tabular}

${ }^{a}$ Unless specified otherwise, reactions were carried out using $\mathbf{1 a}(0.2 \mathrm{mmol})$ under argon. ${ }^{\mathrm{b}}$ Yield determined by ${ }^{19} \mathrm{~F}$ NMR using benzotrifluoride as internal standard. ${ }^{\mathrm{C}}$ Isolated yield in parentheses. ${ }^{\mathrm{d}}$ Yield loss due to volatile product during isolation. ${ }^{\mathrm{e}} 40{ }^{\circ} \mathrm{C}$. ${ }^{\mathrm{f}} 0.4 \mathrm{mmol}$ scale. 


\section{Characterization data:}<smiles>Cc1ccc(SS(=O)(=O)c2ccc(C)cc2)cc1</smiles>

1a: $S$-( $p$-tolyl) 4-methylbenzenesulfonothioate. Prepared according to the general procedure B. Corresponding sulfonyl chloride $(1.91 \mathrm{~g}, 10 \mathrm{mmol})$ was converted to $1 \mathrm{a}$ as a white solid $(3.66 \mathrm{mmol}, 1.02 \mathrm{~g}, 73 \%), \mathrm{R}_{\mathrm{f}}=0.4$ (hexane/EA = 8:1). ${ }^{1} \mathbf{H}$ NMR $\left(500 \mathrm{MHz}, \mathrm{CDCl}_{3}\right): \delta 7.45(\mathrm{~d}, J=8.1 \mathrm{~Hz}, 2 \mathrm{H}), 7.22(\mathrm{dd}, J=12.4 \mathrm{~Hz}, J=8.1 \mathrm{~Hz}$, 4H), $7.14(\mathrm{~d}, J=7.8 \mathrm{~Hz}, 2 \mathrm{H}), 2.42(\mathrm{~s}, 3 \mathrm{H}), 2.37$ (s, 3H) ppm. ${ }^{13} \mathbf{C}$ NMR $\left(126 \mathrm{MHz}, \mathrm{CDCl}_{3}\right): \delta$ 144.7, 142.1, $140.4,136.5,130.3,129.4,127.6,124.6,21.7,21.6 \mathrm{ppm}$. The spectral data are in full accordance with the literature report. ${ }^{1}$<smiles>COc1ccc(SS(=O)(=O)c2ccc(OC)cc2)cc1</smiles>

1b: $S$-(4-methoxyphenyl) 4-methoxybenzenesulfonothioate. Prepared according to the general procedure B. Corresponding sulfonyl chloride $(2.07 \mathrm{~g}, 10 \mathrm{mmol})$ was converted to $\mathbf{1 b}$ as a white solid $(2.76 \mathrm{mmol}, 856.7 \mathrm{mg}$, $56 \%), \mathrm{R}_{\mathrm{f}}=0.5$ (hexane/EA = 4:1). ${ }^{1} \mathbf{H}$ NMR $\left(400 \mathrm{MHz}, \mathrm{CDCl}_{3}\right): \delta 7.52-7.48(\mathrm{~m}, 2 \mathrm{H}), 7.29-7.25(\mathrm{~m}, 2 \mathrm{H})$, $6.89-6.83(\mathrm{~m}, 4 \mathrm{H}), 3.87(\mathrm{~s}, 3 \mathrm{H}), 3.83(\mathrm{~s}, 3 \mathrm{H}) \mathrm{ppm} .{ }^{13} \mathbf{C}$ NMR $\left(126 \mathrm{MHz}, \mathrm{CDCl}_{3}\right)$ : $\delta$ 163.6, 162.2, 138.4, 134.8, $129.9,118.9,115.0,113.9,55.8,55.53 \mathrm{ppm}$. The spectral data are in full accordance with the literature report. $^{1}$<smiles>CC(C)(C)c1ccc(SS(=O)(=O)c2ccc(C(C)(C)C)cc2)cc1</smiles>

1d: $S$-(4-(tert-butyl)phenyl) 4-(tert-butyl)benzenesulfonothioate. Prepared according to the general procedure B. Corresponding sulfonyl chloride $(465.5 \mathrm{mg}, 2.0 \mathrm{mmol})$ was converted to $\mathbf{1 d}$ as a white solid $(0.79 \mathrm{mmol}$, $285.2 \mathrm{mg}, 79 \%), \mathrm{R}_{\mathrm{f}}=0.4$ (hexane/EA = 10:1). ${ }^{1} \mathbf{H} \mathbf{~ N M R}\left(500 \mathrm{MHz}, \mathrm{CDCl}_{3}\right): \delta 7.48(\mathrm{~s}, 2 \mathrm{H}), 7.41(\mathrm{~s}, 2 \mathrm{H}), 7.33$ $(\mathrm{s}, 2 \mathrm{H}), 7.28(\mathrm{~s}, 2 \mathrm{H}), 1.33(\mathrm{~s}, 9 \mathrm{H}), 1.32(\mathrm{~s}, 9 \mathrm{H}) \mathrm{ppm} .{ }^{13} \mathbf{C}$ NMR $\left(126 \mathrm{MHz}, \mathrm{CDCl}_{3}\right): \delta 157.7,155.1,140.3,136.4$, $127.6,126.6,125.8,124.7,35.4,35.0,31.3,31.2 \mathrm{ppm}$. The spectral data are in full accordance with the literature report. $^{2 \mathrm{a}}$<smiles>N#Cc1ccc(SS(=O)(=O)c2ccc(C#N)cc2)cc1</smiles>

1e: $S$-(4-cyanophenyl) 4-cyanobenzenesulfonothioate. Prepared according to the general procedure B. Corresponding sulfonyl chloride $(403.3 \mathrm{mg}, 2.0 \mathrm{mmol})$ was converted to 1e as a yellow solid $(0.47 \mathrm{mmol}, 142.3$ $\mathrm{mg}, 47 \%), \mathrm{R}_{\mathrm{f}}=0.3$ (hexane/EA = 4:1). ${ }^{1} \mathbf{H} \mathbf{N M R}\left(400 \mathrm{MHz}, \mathrm{CDCl}_{3}\right): \delta 7.78(\mathrm{~d}, J=8.5 \mathrm{~Hz}, 2 \mathrm{H}), 7.70(\mathrm{t}, J=8.0$ $\mathrm{Hz}, 4 \mathrm{H}), 7.54(\mathrm{~d}, J=8.3 \mathrm{~Hz}, 2 \mathrm{H}) \mathrm{ppm} .{ }^{13} \mathbf{C} \mathbf{N M R}\left(101 \mathrm{MHz}, \mathrm{CDCl}_{3}\right): \delta 146.5,136.9,133.2,133.1,132.7$, 128.0, 118.0, 117.4, 116.9, 115.9 ppm. HRMS m/z (APCI): calcd. for $\mathrm{C}_{14} \mathrm{H}_{7} \mathrm{~N}_{2} \mathrm{O}_{2} \mathrm{~S}_{2}[\mathrm{M}-\mathrm{H}]^{-}$: 298.9954; found: 298.9957.<smiles>CC(=O)c1ccc(SS(=O)(=O)c2ccc(C(C)=O)cc2)cc1</smiles>

1f: dimethyl 4-((4-benzoatethio)sulfonyl)benzoate. Prepared according to the general procedure $B$. Corresponding sulfonyl chloride $(469.3 \mathrm{mg}, 2.0 \mathrm{mmol})$ was converted to $\mathbf{1 f}$ as a yellow solid $(0.58 \mathrm{mmol}, 213.8$ $\mathrm{mg}, 58 \%), \mathrm{R}_{\mathrm{f}}=0.3$ (hexane/EA = 4:1). ${ }^{1} \mathbf{H}$ NMR (400 MHz, $\left.\mathrm{CDCl}_{3}\right): \delta 8.08(\mathrm{~d}, J=8.4 \mathrm{~Hz}, 2 \mathrm{H}), 7.99(\mathrm{~d}, J=$ $8.2 \mathrm{~Hz}, 2 \mathrm{H}), 7.62(\mathrm{~d}, J=8.4 \mathrm{~Hz}, 2 \mathrm{H}), 7.44(\mathrm{~d}, J=8.2 \mathrm{~Hz}, 2 \mathrm{H}), 3.96(\mathrm{~s}, 3 \mathrm{H}), 3.94(\mathrm{~s}, 3 \mathrm{H}) \mathrm{ppm} .{ }^{13} \mathbf{C}$ NMR $(101$ $\left.\mathrm{MHz}, \mathrm{CDCl}_{3}\right): \delta 166.0,165.4,146.4,136.4,134.9,133.0,132.5,130.6,130.3,127.6,52.9,52.7 \mathrm{ppm}$. The spectral data are in full accordance with the literature report. ${ }^{2 a}$ 
<smiles>O=S(=O)(Sc1ccc(Cl)cc1)c1ccc(Cl)cc1</smiles>

1g: $S$-(4-chlorophenyl) 4-chlorobenzenesulfonothioate. Prepared according to the general procedure B. Corresponding sulfonyl chloride $(422.1 \mathrm{mg}, 2.0 \mathrm{mmol})$ was converted to $\mathbf{1 g}$ as a white solid $(0.60 \mathrm{mmol}, 193.1$ $\mathrm{mg}, 60 \%), \mathrm{R}_{\mathrm{f}}=0.4$ (hexane/EA = 10:1). ${ }^{1} \mathbf{H} \mathbf{~ N M R}\left(500 \mathrm{MHz}, \mathrm{CDCl}_{3}\right): \delta 7.52(\mathrm{~d}, J=8.6 \mathrm{~Hz}, 2 \mathrm{H}), 7.43(\mathrm{~d}, J=$ $8.6 \mathrm{~Hz}, 2 \mathrm{H}), 7.35(\mathrm{~d}, J=8.5 \mathrm{~Hz}, 2 \mathrm{H}), 7.31(\mathrm{~d}, J=8.5 \mathrm{~Hz}, 2 \mathrm{H}) \mathrm{ppm} .{ }^{13} \mathbf{C} \mathbf{N M R}\left(126 \mathrm{MHz}, \mathrm{CDCl}_{3}\right): \delta 141.4$, $140.7,138.7,137.8,130.0,129.4,129.0,126.1 \mathrm{ppm}$. The spectral data are in full accordance with the literature report. ${ }^{1}$<smiles>O=S(=O)(Sc1ccc(Br)cc1)c1ccc(Br)cc1</smiles>

1h: $S$-(4-bromophenyl) 4-bromobenzenesulfonothioate. Prepared according to the general procedure B. Corresponding sulfonyl chloride $(511.0 \mathrm{mg}, 2.0 \mathrm{mmol})$ was converted to $\mathbf{1 h}$ as a white solid $(0.56 \mathrm{mmol}, 227.9$ $\mathrm{mg}, 56 \%), \mathrm{R}_{\mathrm{f}}=0.4$ (hexane/EA = 10:1). ${ }^{1} \mathbf{H} \mathbf{N M R}\left(500 \mathrm{MHz}, \mathrm{CDCl}_{3}\right): \delta 7.62-7.58(\mathrm{~m}, 2 \mathrm{H}), 7.53-7.50(\mathrm{~m}$, $2 \mathrm{H}), 7.46-7.42(\mathrm{~m}, 2 \mathrm{H}), 7.25-7.23(\mathrm{~m}, 2 \mathrm{H}) \mathrm{ppm} .{ }^{13} \mathbf{C}$ NMR $\left(126 \mathrm{MHz}, \mathrm{CDCl}_{3}\right): \delta$ 142.0, 138.0, 133.0, 132.4, $129.3,129.1,127.1,126.7 \mathrm{ppm}$. The spectral data are in full accordance with the literature report. ${ }^{1}$<smiles>O=S(=O)(Sc1cccc(Br)c1)c1cccc(Br)c1</smiles>

1i: $S$-(3-bromophenyl) 3-bromobenzenesulfonothioate. Prepared according to the general procedure B. Corresponding sulfonyl chloride $(511.0 \mathrm{mg}, 2.0 \mathrm{mmol})$ was converted to $\mathbf{1 i}$ as a white solid $(0.33 \mathrm{mmol}, 135.6$ $\mathrm{mg}, 33 \%), \mathrm{R}_{\mathrm{f}}=0.3$ (hexane/EA = 10:1). ${ }^{1} \mathbf{H} \mathbf{~ N M R}\left(400 \mathrm{MHz}, \mathrm{CDCl}_{3}\right): \delta 7.75$ (ddd, $J=7.9 \mathrm{~Hz}, J=1.8 \mathrm{~Hz}, J=$ $1.0 \mathrm{~Hz}, 1 \mathrm{H}), 7.67(\mathrm{t}, J=1.8 \mathrm{~Hz}, 1 \mathrm{H}), 7.65(\mathrm{ddd}, J=7.9 \mathrm{~Hz}, 1.8 \mathrm{~Hz}, 1.1 \mathrm{~Hz}, 1 \mathrm{H}), 7.54-7.48(\mathrm{~m}, 1 \mathrm{H}), 7.46(\mathrm{t}, J$ $=1.7 \mathrm{~Hz}, 1 \mathrm{H}), 7.35(\mathrm{t}, J=7.9 \mathrm{~Hz}, 2 \mathrm{H}), 7.28(\mathrm{t}, J=7.5 \mathrm{~Hz}, 1 \mathrm{H}) \mathrm{ppm} .{ }^{13} \mathbf{C} \mathbf{N M R}\left(101 \mathrm{MHz}, \mathrm{CDCl}_{3}\right): \delta 144.1$, $139.0,137.1,135.3,134.9,131.0,130.5,129.4,126.1,123.1 \mathrm{ppm}$. The spectral data are in full accordance with the literature report. ${ }^{2 \mathrm{a}}$<smiles>O=S(=O)(Sc1cccs1)c1cccs1</smiles>

1j: $S$-(thiophen-2-yl) thiophene-2-sulfonothioate. Prepared according to the general procedure B. Corresponding sulfonyl chloride $(913.3 \mathrm{mg}, 5.0 \mathrm{mmol})$ was converted to $\mathbf{1 j}$ as a yellow solid $(1.43 \mathrm{mmol}, 375.7$ $\mathrm{mg}, 57 \%), \mathrm{R}_{\mathrm{f}}=0.2$ (hexane/EA = 4:1). ${ }^{1} \mathbf{H}$ NMR $\left(500 \mathrm{MHz}, \mathrm{CDCl}_{3}\right): \delta 7.69(\mathrm{~d}, J=4.9 \mathrm{~Hz}, 1 \mathrm{H}), 7.65(\mathrm{~d}, J=$ $5.3 \mathrm{~Hz}, 1 \mathrm{H}), 7.40(\mathrm{~d}, J=3.8 \mathrm{~Hz}, 1 \mathrm{H}), 7.24(\mathrm{~d}, J=3.7 \mathrm{~Hz}, 1 \mathrm{H}), 7.11(\mathrm{t}, J=4.5 \mathrm{~Hz}, 1 \mathrm{H}), 7.06(\mathrm{t}, J=4.4 \mathrm{~Hz}, 1 \mathrm{H})$ ppm. ${ }^{13} \mathbf{C}$ NMR $\left(126 \mathrm{MHz}, \mathrm{CDCl}_{3}\right): \delta 142.6,139.8,135.7,134.7,134.6,128.6,127.4,125.3 \mathrm{ppm}$. The spectral data are in full accordance with the literature report. ${ }^{1}$<smiles>O=S(=O)(Sc1cccc2ccccc12)c1cccc2ccccc12</smiles>

1k: $S$-(naphthalen-1-yl) naphthalene-1-sulfonothioate. Prepared according to the general procedure B. Corresponding sulfonyl chloride $(453.4 \mathrm{mg}, 2.0 \mathrm{mmol})$ was converted to $\mathbf{1 k}$ as a yellow solid $(0.17 \mathrm{mmol}, 61.0$ $\mathrm{mg}, 17 \%), \mathrm{R}_{\mathrm{f}}=0.2$ (hexane/EA = 10:1). ${ }^{1} \mathbf{H} \mathbf{N M R}\left(500 \mathrm{MHz}, \mathrm{CDCl}_{3}\right): \delta 8.85(\mathrm{~d}, J=8.5 \mathrm{~Hz}, 1 \mathrm{H}), 7.90(\mathrm{~d}, J=$ $6.4 \mathrm{~Hz}, 3 \mathrm{H}), 7.72(\mathrm{t}, J=7.5 \mathrm{~Hz}, 2 \mathrm{H}), 7.65(\mathrm{t}, J=9.6 \mathrm{~Hz}, 2 \mathrm{H}), 7.54(\mathrm{~d}, J=6.9 \mathrm{~Hz}, 1 \mathrm{H}), 7.48(\mathrm{~d}, J=7.2 \mathrm{~Hz}, 1 \mathrm{H})$, $7.34(\mathrm{t}, J=7.4 \mathrm{~Hz}, 2 \mathrm{H}), 7.09-7.03(\mathrm{~m}, 2 \mathrm{H}) \mathrm{ppm} .{ }^{13} \mathbf{C} \mathbf{N M R}\left(126 \mathrm{MHz}, \mathrm{CDCl}_{3}\right): \delta 138.0,137.1,135.4,134.5$, $134.4,134.0,132.8,130.7,129.0,128.7,128.3,128.0,127.2,127.2,126.6,125.7,125.5,125.1,124.8,123.5$ ppm. The spectral data are in full accordance with the literature report. ${ }^{1}$<smiles>O=S(=O)(Sc1ccc2c(c1)CCO2)c1ccc2c(c1)CCO2</smiles>

11: $S$-(2,3-dihydrobenzofuran-5-yl) 2,3-dihydrobenzofuran-5-sulfonothioate. Prepared according to the general procedure B. Corresponding sulfonyl chloride $(437.3 \mathrm{mg}, 2.0 \mathrm{mmol})$ was converted to $\mathbf{1 l}$ as a white solid (0.39 mmol, $129.3 \mathrm{mg}, 39 \%), \mathrm{R}_{\mathrm{f}}=0.2$ (hexane/EA $\left.=4: 1\right) .{ }^{1} \mathbf{H}$ NMR $\left(400 \mathrm{MHz}, \mathrm{CDCl}_{3}\right): \delta 7.46(\mathrm{~s}, 1 \mathrm{H})$, $7.33-7.29(\mathrm{~m}, 2 \mathrm{H}), 6.99(\mathrm{dd}, J=8.3 \mathrm{~Hz}, J=1.8 \mathrm{~Hz}, 1 \mathrm{H}), 6.71(\mathrm{dd}, J=14.9 \mathrm{~Hz}, J=8.4 \mathrm{~Hz}, 2 \mathrm{H}), 4.67(\mathrm{dt}, J=$ 
$20.3 \mathrm{~Hz}, J=8.8 \mathrm{~Hz}, 4 \mathrm{H}), 3.22(\mathrm{td}, J=8.8 \mathrm{~Hz}, J=4.4 \mathrm{~Hz}, 4 \mathrm{H}) \mathrm{ppm} .{ }^{13} \mathbf{C}$ NMR $\left(101 \mathrm{MHz}, \mathrm{CDCl}_{3}\right): \delta 164.6$, 163.2, 137.6, 135.0, 133.9, 129.9, 129.0, 128.2, 125.0, 118.6, 110.2, 109.1, 72.6, 72.2, 29.3, 29.0 ppm. HRMS $\mathrm{m} / \mathrm{z}$ (APCI): calcd. for $\mathrm{C}_{16} \mathrm{H}_{15} \mathrm{O}_{4} \mathrm{~S}_{2}[\mathrm{M}+\mathrm{H}]^{+}: 335.0406$; found: 335.0409 .<smiles>Cc1ccc(S(=O)(=O)SCCCCCOC(=O)c2ccccc2)cc1</smiles>

1m: 5-(tosylthio)pentyl benzoate. Prepared according to the general procedure $\mathrm{C}$. Corresponding alkyl bromide $(774.6 \mathrm{mg}, 2.9 \mathrm{mmol})$ was converted to $\mathbf{1 m}$ as a white solid $(2.1 \mathrm{mmol}, 801.6 \mathrm{mg}, 89 \%), \mathrm{R}_{\mathrm{f}}=0.4$ (hexane/EA = 4:1). ${ }^{1} \mathbf{H}$ NMR $\left(400 \mathrm{MHz}, \mathrm{CDCl}_{3}\right): \delta 8.05(\mathrm{~d}, J=7.6 \mathrm{~Hz}, 2 \mathrm{H}), 7.82(\mathrm{~d}, J=8.1 \mathrm{~Hz}, 2 \mathrm{H}), 7.58(\mathrm{t}, J$ $=7.3 \mathrm{~Hz}, 1 \mathrm{H}), 7.46(\mathrm{t}, J=7.6 \mathrm{~Hz}, 2 \mathrm{H}), 7.33(\mathrm{~d}, J=8.1 \mathrm{~Hz}, 2 \mathrm{H}), 4.27(\mathrm{t}, J=6.4 \mathrm{~Hz}, 2 \mathrm{H}), 3.03(\mathrm{t}, J=7.2 \mathrm{~Hz}$, 2H), $2.43(\mathrm{~s}, 3 \mathrm{H}), 1.76-1.65(\mathrm{~m}, 4 \mathrm{H}), 1.51-1.43(\mathrm{~m}, 2 \mathrm{H}) \mathrm{ppm} .{ }^{13} \mathbf{C}$ NMR $\left(101 \mathrm{MHz}, \mathrm{CDCl}_{3}\right): \delta 166.3,144.7$, 141.8, 132.8, 130.1, 129.7, 129.4, 128.3, 126.8, 64.3, 35.7, 28.2, 27.9, 24.9, 21.5 ppm. HRMS m/z (ESI): calcd. for $\mathrm{C}_{19} \mathrm{H}_{22} \mathrm{O}_{4} \mathrm{~S}_{2} \mathrm{Na}[\mathrm{M}+\mathrm{Na}]^{+}$: 401.0852 ; found: 401.0850 .<smiles>Cc1ccc(S(=O)(=O)SCCOC(=O)c2ccccc2)cc1</smiles>

1n: 2-(tosylthio)ethyl benzoate. Prepared according to the general procedure C. Corresponding alkyl bromide (716.5 mg, $3.1 \mathrm{mmol}$ ) was converted to $\mathbf{1 n}$ as a white solid $(0.49 \mathrm{mmol}, 164.7 \mathrm{mg}, 19 \%), \mathrm{R}_{\mathrm{f}}=0.4$ (hexane/EA = 4:1). ${ }^{1} \mathbf{H}$ NMR $\left(400 \mathrm{MHz}, \mathrm{CDCl}_{3}\right): \delta 7.97(\mathrm{~d}, J=7.3 \mathrm{~Hz}, 2 \mathrm{H}), 7.83(\mathrm{~d}, J=8.3 \mathrm{~Hz}, 2 \mathrm{H}), 7.56(\mathrm{t}, J=7.4 \mathrm{~Hz}$, $1 \mathrm{H}), 7.42(\mathrm{t}, J=7.7 \mathrm{~Hz}, 2 \mathrm{H}), 7.32(\mathrm{~d}, J=8.1 \mathrm{~Hz}, 2 \mathrm{H}), 4.47(\mathrm{t}, J=6.4 \mathrm{~Hz}, 2 \mathrm{H}), 3.36(\mathrm{t}, J=6.4 \mathrm{~Hz}, 2 \mathrm{H}), 2.42(\mathrm{~s}$, $3 \mathrm{H}) \mathrm{ppm} .{ }^{13} \mathrm{C}$ NMR $\left(101 \mathrm{MHz}, \mathrm{CDCl}_{3}\right): \delta 166.0,145.2,141.8,133.3,130.0,129.7,129.5,128.5,127.1,62.3$, 34.5, 21.7 ppm. HRMS m/z (ESI): calcd. for $\mathrm{C}_{16} \mathrm{H}_{16} \mathrm{O}_{4} \mathrm{~S}_{2} \mathrm{Na}[\mathrm{M}+\mathrm{Na}]^{+}$: 359.0382; found: 359.0380 .<smiles>Cc1ccc(S(=O)(=O)SCCCCCOC(=O)CCc2ccccc2)cc1</smiles>

1o: 5-(tosylthio)pentyl 3-phenylpropanoate. Prepared according to the general procedure C. Corresponding alkyl bromide (559.5 mg, $1.9 \mathrm{mmol})$ was converted to 10 as a colorless oil $(1.4 \mathrm{mmol}, 569.6 \mathrm{mg}, 90 \%), \mathrm{R}_{\mathrm{f}}=0.4$ (hexane/EA = 4:1). ${ }^{1} \mathbf{H}$ NMR $\left(400 \mathrm{MHz}, \mathrm{CDCl}_{3}\right): \delta 7.83(\mathrm{~d}, J=8.3 \mathrm{~Hz}, 2 \mathrm{H}), 7.36(\mathrm{~d}, J=8.2 \mathrm{~Hz}, 2 \mathrm{H}), 7.33-$ $7.27(\mathrm{~m}, 2 \mathrm{H}), 7.24-7.19(\mathrm{~m}, 3 \mathrm{H}), 4.02(\mathrm{t}, J=6.5 \mathrm{~Hz}, 2 \mathrm{H}), 2.97(\mathrm{dd}, J=14.3,7.2 \mathrm{~Hz}, 4 \mathrm{H}), 2.64(\mathrm{t}, J=7.8 \mathrm{~Hz}$, $2 \mathrm{H}), 2.46(\mathrm{~s}, 3 \mathrm{H}), 1.67-1.50(\mathrm{~m}, 4 \mathrm{H}), 1.37-1.27(\mathrm{~m}, 2 \mathrm{H}) \mathrm{ppm} .{ }^{13} \mathbf{C}$ NMR $\left(101 \mathrm{MHz}, \mathrm{CDCl}_{3}\right): \delta 172.8,144.8$, $141.9,140.4,129.8,128.4,128.2,126.9,126.2,63.9,35.8,35.7,30.9,28.2,27.9,24.8,21.6 \mathrm{ppm}$. HRMS m/z (ESI): calcd. for $\mathrm{C}_{21} \mathrm{H}_{26} \mathrm{O}_{4} \mathrm{~S}_{2} \mathrm{Na}[\mathrm{M}+\mathrm{Na}]^{+}$: 429.1165; found: 429.1160 .<smiles>Cc1ccc(S(=O)(=O)SCC2CCN(C(=O)OCc3ccccc3)CC2)cc1</smiles>

1p: tert-butyl 4-((tosylthio)methyl)piperidine-1-carboxylate. Prepared according to the general procedure C. Corresponding alkyl bromide $(667.7 \mathrm{mg}, 2.4 \mathrm{mmol})$ was converted to $1 \mathbf{p}$ as a white solid $(0.53 \mathrm{mmol}, 202.7 \mathrm{mg}$, $26 \%), \mathrm{R}_{\mathrm{f}}=0.3$ (hexane/EA = 10:1). ${ }^{1} \mathbf{H}$ NMR $\left(400 \mathrm{MHz}, \mathrm{CDCl}_{3}\right): \delta 7.78(\mathrm{~d}, J=8.1 \mathrm{~Hz}, 2 \mathrm{H}), 7.33(\mathrm{~d}, J=8.1$ $\mathrm{Hz}, 2 \mathrm{H}), 4.04(\mathrm{~d}, J=13.1 \mathrm{~Hz}, 2 \mathrm{H}), 2.87(\mathrm{~d}, J=6.6 \mathrm{~Hz}, 2 \mathrm{H}), 2.57(\mathrm{t}, J=12.4 \mathrm{~Hz}, 2 \mathrm{H}), 2.43(\mathrm{~s}, 3 \mathrm{H}), 1.66(\mathrm{~d}, J=$ $10.2 \mathrm{~Hz}, 3 \mathrm{H}), 1.41(\mathrm{~s}, 9 \mathrm{H}), 1.04(\mathrm{ddd}, J=16.6 \mathrm{~Hz}, J=12.8 \mathrm{~Hz}, J=4.2 \mathrm{~Hz}, 2 \mathrm{H}) \mathrm{ppm} .{ }^{13} \mathbf{C} \mathbf{~ N M R}(101 \mathrm{MHz}$, $\left.\mathrm{CDCl}_{3}\right): \delta 154.6,144.8,141.8,129.8,126.9,79.5,43.4,41.7,35.7,31.2,28.3,21.6 \mathrm{ppm}$. HRMS m/z (ESI): calcd. for $\mathrm{C}_{18} \mathrm{H}_{27} \mathrm{NO}_{4} \mathrm{~S}_{2} \mathrm{Na}[\mathrm{M}+\mathrm{Na}]^{+}$: 408.1274 ; found: 408.1272 .<smiles>Cc1ccc(S(=O)(=O)SCCCCCN2C(=O)c3ccccc3C2=O)cc1</smiles>

1q: S-(5-(1,3-dioxoisoindolin-2-yl)pentyl) 4-methylbenzenesulfonothioate. Prepared according to the general procedure C. Corresponding alkyl bromide $(1.05 \mathrm{~g}, 3.5 \mathrm{mmol})$ was converted to $\mathbf{1 q}$ as a white solid $(2.5 \mathrm{mmol}$, $999.9 \mathrm{mg}, 83 \%), \mathrm{R}_{\mathrm{f}}=0.2$ (hexane/EA = 4:1). ${ }^{1} \mathbf{H}$ NMR $\left(400 \mathrm{MHz}, \mathrm{CDCl}_{3}\right): \delta 7.84(\mathrm{dd}, J=5.5 \mathrm{~Hz}, J=3.0 \mathrm{~Hz}$, $2 \mathrm{H}), 7.80(\mathrm{~d}, J=8.3 \mathrm{~Hz}, 2 \mathrm{H}), 7.72(\mathrm{dd}, J=5.4,3.1 \mathrm{~Hz}, 2 \mathrm{H}), 7.33(\mathrm{~d}, J=8.0 \mathrm{~Hz}, 2 \mathrm{H}), 3.62(\mathrm{t}, J=7.2 \mathrm{~Hz}, 2 \mathrm{H})$, $2.96(\mathrm{t}, J=7.4 \mathrm{~Hz}, 2 \mathrm{H}), 2.44(\mathrm{~s}, 3 \mathrm{H}), 1.69-1.57(\mathrm{~m}, 4 \mathrm{H}), 1.39-1.30(\mathrm{~m}, 2 \mathrm{H}) \mathrm{ppm} .{ }^{13} \mathbf{C} \mathbf{N M R}(126 \mathrm{MHz}$, $\left.\mathrm{CDCl}_{3}\right): \delta 168.3,144.7,142.0,134.0,132.0,129.9,127.0,123.2,37.5,35.7,28.2,27.9,25.7,21.7$ ppm. HRMS $\mathrm{m} / \mathrm{z}$ (ESI): calcd. for $\mathrm{C}_{20} \mathrm{H}_{21} \mathrm{NO}_{4} \mathrm{~S}_{2} \mathrm{Na}[\mathrm{M}+\mathrm{Na}]^{+}$: 426.0804 ; found: 426.0803 . 
<smiles>Cc1ccc(S(=O)(=O)CCCCCO[Si](c2ccccc2)(c2ccccc2)C(C)(C)C)cc1</smiles>

1r: $S$-(5-((tert-butyldiphenylsilyl)oxy)pentyl) 4-methylbenzenesulfonothioate. Prepared according to the general procedure C. Corresponding alkyl bromide $(506.8 \mathrm{mg}, 1.3 \mathrm{mmol})$ was converted to $\mathbf{1 r}$ as a colorless oil $(0.73 \mathrm{mmol}, 375.4 \mathrm{mg}, 70 \%), \mathrm{R}_{\mathrm{f}}=0.6$ (hexane/EA = 4:1). ${ }^{1} \mathbf{H} \mathbf{N M R}\left(400 \mathrm{MHz}, \mathrm{CDCl}_{3}\right): \delta 7.83(\mathrm{~d}, J=8.3 \mathrm{~Hz}$, 2H), $7.68(\mathrm{dd}, J=7.8,1.6 \mathrm{~Hz}, 4 \mathrm{H}), 7.48-7.37(\mathrm{~m}, 6 \mathrm{H}), 7.34(\mathrm{~d}, J=8.2 \mathrm{~Hz}, 2 \mathrm{H}), 3.63(\mathrm{t}, J=6.2 \mathrm{~Hz}, 2 \mathrm{H}), 2.98$ $(\mathrm{t}, J=7.4 \mathrm{~Hz}, 2 \mathrm{H}), 2.44(\mathrm{~s}, 3 \mathrm{H}), 1.65-1.56(\mathrm{~m}, 2 \mathrm{H}), 1.55-1.47(\mathrm{~m}, 2 \mathrm{H}), 1.45-1.37(\mathrm{~m}, 2 \mathrm{H}), 1.07(\mathrm{~s}, 9 \mathrm{H})$ ppm. ${ }^{13} \mathrm{C}$ NMR $\left(101 \mathrm{MHz}, \mathrm{CDCl}_{3}\right): \delta 144.7,142.1,135.6,133.9,129.9,129.7,127.7,127.0,63.4,36.0,31.8$, 28.4, 26.9, 24.9, 21.7, 19.2 ppm. HRMS m/z (ESI): calcd. for $\mathrm{C}_{28} \mathrm{H}_{36} \mathrm{O}_{3} \mathrm{~S}_{2} \mathrm{SiNa}[\mathrm{M}+\mathrm{Na}]^{+}$: 535.1767; found: 535.1768 .<smiles>Cc1ccc(S(=O)(=O)SCCCc2ccccc2)cc1</smiles>

1s: $S$-(3-phenylpropyl) 4-methylbenzenesulfonothioate. Prepared according to the general procedure C. Corresponding alkyl bromide $(477.8 \mathrm{mg}, 2.4 \mathrm{mmol})$ was converted to $1 \mathrm{~s}$ as a yellow solid (1.7 mmol, $525.1 \mathrm{mg}$, $86 \%), \mathrm{R}_{\mathrm{f}}=0.2$ (hexane/EA = 10:1). ${ }^{1} \mathbf{H}$ NMR $\left(400 \mathrm{MHz}, \mathrm{CDCl}_{3}\right): \delta 7.78(\mathrm{~d}, J=8.3 \mathrm{~Hz}, 2 \mathrm{H}), 7.33(\mathrm{~d}, J=8.1$ $\mathrm{Hz}, 2 \mathrm{H}), 7.28(\mathrm{t}, J=7.2 \mathrm{~Hz}, 2 \mathrm{H}), 7.21(\mathrm{t}, J=7.3 \mathrm{~Hz}, 1 \mathrm{H}), 7.08(\mathrm{~d}, J=7.1 \mathrm{~Hz}, 2 \mathrm{H}), 2.98(\mathrm{t}, J=7.3 \mathrm{~Hz}, 2 \mathrm{H}), 2.64$ $(\mathrm{t}, J=7.5 \mathrm{~Hz}, 2 \mathrm{H}), 2.46(\mathrm{~s}, 3 \mathrm{H}), 2.00-1.90(\mathrm{~m}, 2 \mathrm{H}) \mathrm{ppm} .{ }^{13} \mathbf{C} \mathbf{N M R}\left(126 \mathrm{MHz}, \mathrm{CDCl}_{3}\right): \delta 144.8,141.9,140.3$, 129.9, 128.5, 128.4, 127.0, 126.2, 35.2, 34.4, 30.2, 21.7 pm. HRMS m/z (ESI): calcd. for $\mathrm{C}_{16} \mathrm{H}_{18} \mathrm{O}_{2} \mathrm{~S}_{2} \mathrm{Na}$ $[\mathrm{M}+\mathrm{Na}]^{+}: 329.0640$; found: 329.0638 .<smiles>Cc1ccc(S(=O)(=O)SCCc2ccccc2)cc1</smiles>

1t: $S$-phenethyl 4-methylbenzenesulfonothioate. Prepared according to the general procedure C. Corresponding alkyl bromide $(444.1 \mathrm{mg}, 2.4 \mathrm{mmol})$ was converted to $\mathbf{1 t}$ as a yellow oil (1.0 mmol, $299.0 \mathrm{mg}$, $51 \%), \mathrm{R}_{\mathrm{f}}=0.2$ (hexane/EA = 10:1). ${ }^{\mathbf{1}} \mathbf{H} \mathbf{N M R}\left(400 \mathrm{MHz}, \mathrm{CDCl}_{3}\right): \delta 7.84(\mathrm{~d}, J=8.4 \mathrm{~Hz}, 2 \mathrm{H}), 7.36(\mathrm{~d}, J=8.0$ $\mathrm{Hz}, 2 \mathrm{H}), 7.30(\mathrm{t}, J=7.1 \mathrm{~Hz}, 2 \mathrm{H}), 7.24(\mathrm{t}, J=7.3 \mathrm{~Hz}, 1 \mathrm{H}), 7.12(\mathrm{~d}, J=6.8 \mathrm{~Hz}, 2 \mathrm{H}), 3.24(\mathrm{t}, J=7.7 \mathrm{~Hz}, 2 \mathrm{H}), 2.92$ $(\mathrm{t}, J=7.7 \mathrm{~Hz}, 2 \mathrm{H}), 2.47$ (s, 3H) ppm. ${ }^{13} \mathbf{C}$ NMR $\left(126 \mathrm{MHz}, \mathrm{CDCl}_{3}\right): \delta 144.9,141.9,138.7,129.9,128.6,128.5$, $127.0,126.8,37.1,35.1,21.7 \mathrm{ppm}$. The spectral data are in full accordance with the literature report. ${ }^{6}$<smiles>CCC(SS(=O)(=O)c1ccc(C)cc1)c1ccccc1</smiles>

1u: $S$-(1-phenylpropyl) 4-methylbenzenesulfonothioate. Prepared according to the general procedure C. Corresponding alkyl bromide $(318.0 \mathrm{mg}, 1.6 \mathrm{mmol})$ was converted to $\mathbf{1 u}$ as a white solid $(1.1 \mathrm{mmol}, 328.1 \mathrm{mg}$, $82 \%), \mathrm{R}_{\mathrm{f}}=0.3$ (hexane/EA = 10:1). ${ }^{1} \mathbf{H}$ NMR $\left(400 \mathrm{MHz}, \mathrm{CDCl}_{3}\right): \delta 7.49(\mathrm{~d}, J=8.3 \mathrm{~Hz}, 2 \mathrm{H}), 7.18-7.06(\mathrm{~m}$, $7 \mathrm{H}), 4.36(\mathrm{dd}, J=9.0,6.4 \mathrm{~Hz}, 1 \mathrm{H}), 2.36(\mathrm{~s}, 3 \mathrm{H}), 2.08-1.83(\mathrm{~m}, 2 \mathrm{H}), 0.86(\mathrm{t}, J=7.3 \mathrm{~Hz}, 3 \mathrm{H}) \mathrm{ppm} .{ }^{13} \mathbf{C}$ NMR (126 MHz, $\left.\mathrm{CDCl}_{3}\right): \delta 144.1,142.5,139.3,129.4,128.6,127.8,127.6,126.8,57.6,29.8,21.6,12.0$ ppm. HRMS $\mathrm{m} / \mathrm{z}$ (ESI): calcd. for $\mathrm{C}_{16} \mathrm{H}_{18} \mathrm{O}_{2} \mathrm{~S}_{2} \mathrm{Na}[\mathrm{M}+\mathrm{Na}]^{+}:$329.0640; found: 329.0640 .<smiles>COc1ccc(CSS(=O)(=O)c2ccc(C)cc2)cc1</smiles>

1v: $S$-(4-methoxybenzyl) 4-methylbenzenesulfonothioate. Prepared according to the general procedure C. Corresponding alkyl bromide $(564.5 \mathrm{mg}, 2.8 \mathrm{mmol})$ was converted to $\mathbf{1 v}$ as a yellow solid (1.2 mmol, $358.1 \mathrm{mg}$, $50 \%), \mathrm{R}_{\mathrm{f}}=0.3$ (hexane/EA = 10:1). ${ }^{1} \mathbf{H}$ NMR $\left(400 \mathrm{MHz}, \mathrm{CDCl}_{3}\right): \delta 7.74(\mathrm{~d}, J=7.0 \mathrm{~Hz}, 2 \mathrm{H}), 7.29(\mathrm{~d}, J=6.9$ $\mathrm{Hz}, 2 \mathrm{H}), 7.10(\mathrm{~d}, J=7.1 \mathrm{~Hz}, 2 \mathrm{H}), 6.76(\mathrm{~d}, J=7.2 \mathrm{~Hz}, 2 \mathrm{H}), 4.21(\mathrm{~s}, 2 \mathrm{H}), 3.77(\mathrm{~s}, 3 \mathrm{H}), 2.45(\mathrm{~s}, 3 \mathrm{H}) \mathrm{ppm} .{ }^{13} \mathrm{C}$ NMR $\left(126 \mathrm{MHz}, \mathrm{CDCl}_{3}\right): \delta 159.4,144.6,142.0,130.4,129.8,127.0,125.3,114.2,55.3,40.0,21.7 \mathrm{ppm}$. The spectral data are in full accordance with the literature report. ${ }^{7}$ 
<smiles>Cc1ccc(S(=O)(=O)Cc2ccc(C(C)(C)C)cc2)cc1</smiles>

1w: $S$-(4-(tert-butyl)benzyl) 4-methylbenzenesulfonothioate. Prepared according to the general procedure C. Corresponding alkyl bromide $(545.1 \mathrm{mg}, 2.4 \mathrm{mmol})$ was converted to $\mathbf{1 w}$ as a yellow solid $(1.1 \mathrm{mmol}, 376.4$ $\mathrm{mg}, 56 \%), \mathrm{R}_{\mathrm{f}}=0.5$ (hexane/EA = 4:1). ${ }^{1} \mathbf{H}$ NMR $\left(400 \mathrm{MHz}, \mathrm{CDCl}_{3}\right): \delta 7.75(\mathrm{~d}, J=8.4 \mathrm{~Hz}, 2 \mathrm{H}), 7.29(\mathrm{~d}, J=$ $2.0 \mathrm{~Hz}, 2 \mathrm{H}), 7.27(\mathrm{~d}, J=2.0 \mathrm{~Hz}, 2 \mathrm{H}), 7.14(\mathrm{~d}, J=8.4 \mathrm{~Hz}, 2 \mathrm{H}), 4.27(\mathrm{~s}, 2 \mathrm{H}), 2.45(\mathrm{~s}, 3 \mathrm{H}), 1.31(\mathrm{~s}, 9 \mathrm{H}) \mathrm{ppm} .{ }^{13} \mathrm{C}$ NMR $\left(101 \mathrm{MHz}, \mathrm{CDCl}_{3}\right): \delta 151.0,144.5,142.1,130.5,129.7,128.8,126.9,125.6,40.0,34.5,31.3,21.6 \mathrm{ppm}$. HRMS m/z (ESI): calcd. for $\mathrm{C}_{18} \mathrm{H}_{22} \mathrm{O}_{2} \mathrm{~S}_{2} \mathrm{Na}[\mathrm{M}+\mathrm{Na}]^{+}$: 357.0953 ; found: 357.0950 .<smiles>Cc1ccc(S(=O)(=O)CSc2ccc(Cl)cc2)cc1</smiles>

1x: $S$-(4-chlorobenzyl) 4-methylbenzenesulfonothioate. Prepared according to the general procedure C. Corresponding alkyl bromide $(493.2 \mathrm{mg}, 2.4 \mathrm{mmol})$ was converted to $\mathbf{1 x}$ as a yellow solid (1.4 mmol, $451.1 \mathrm{mg}$, $72 \%), \mathrm{R}_{\mathrm{f}}=0.3$ (hexane/EA = 4:1). ${ }^{1} \mathbf{H}$ NMR (400 MHz, $\left.\mathrm{CDCl}_{3}\right): \delta 7.65(\mathrm{~d}, J=8.4 \mathrm{~Hz}, 2 \mathrm{H}), 7.23(\mathrm{~d}, J=8.1 \mathrm{~Hz}$, $2 \mathrm{H}), 7.14(\mathrm{~d}, J=8.5 \mathrm{~Hz}, 2 \mathrm{H}), 7.07(\mathrm{~d}, J=8.6 \mathrm{~Hz}, 2 \mathrm{H}), 4.19(\mathrm{~s}, 2 \mathrm{H}), 2.41(\mathrm{~s}, 3 \mathrm{H}) \mathrm{ppm} .{ }^{13} \mathbf{C} \mathbf{N M R}(101 \mathrm{MHz}$, $\mathrm{CDCl}_{3}$ ): $\delta 144.8,141.9,133.7,132.4,130.4,129.7,128.8,126.9,39.5,21.6 \mathrm{ppm}$. HRMS m/z (ESI): calcd. for $\mathrm{C}_{14} \mathrm{H}_{13} \mathrm{ClO}_{2} \mathrm{~S}_{2} \mathrm{Na}[\mathrm{M}+\mathrm{Na}]^{+}$: 334.9938 ; found: 334.9935 .<smiles>Cc1ccc(S(=O)(=O)CSc2ccc(Br)cc2)cc1</smiles>

1y: S-(4-bromobenzyl) 4-methylbenzenesulfonothioate. Prepared according to the general procedure C. Corresponding alkyl bromide $(599.8 \mathrm{mg}, 2.4 \mathrm{mmol})$ was converted to $\mathbf{1 y}$ as a yellow solid $(1.4 \mathrm{mmol}, 508.8 \mathrm{mg}$, $71 \%), \mathrm{R}_{\mathrm{f}}=0.3$ (hexane/EA = 4:1). ${ }^{1} \mathbf{H}$ NMR $\left(400 \mathrm{MHz}, \mathrm{CDCl}_{3}\right): \delta 7.66(\mathrm{~d}, J=8.4 \mathrm{~Hz}, 2 \mathrm{H}), 7.30(\mathrm{~d}, J=8.5 \mathrm{~Hz}$, $2 \mathrm{H}), 7.25(\mathrm{~d}, J=8.1 \mathrm{~Hz}, 2 \mathrm{H}), 7.03(\mathrm{~d}, J=8.5 \mathrm{~Hz}, 2 \mathrm{H}), 4.19(\mathrm{~s}, 2 \mathrm{H}), 2.44(\mathrm{~s}, 3 \mathrm{H}) \mathrm{ppm} .{ }^{13} \mathbf{C} \mathbf{N M R}(101 \mathrm{MHz}$, $\left.\mathrm{CDCl}_{3}\right): \delta 144.8,141.8,132.9,131.7,130.7,129.7,126.9,121.8,39.6,21.6 \mathrm{ppm}$. The spectral data are in full accordance with the literature report. ${ }^{3}$<smiles>Cc1ccc(S(=O)(=O)Cc2ccc([N+](=O)[O-])cc2)cc1</smiles>

1z: $S$-(4-nitrobenzyl) 4-methylbenzenesulfonothioate. Prepared according to the general procedure C. Corresponding alkyl bromide $(518.5 \mathrm{mg}, 2.4 \mathrm{mmol})$ was converted to $\mathbf{1 z}$ as a white solid $(0.69 \mathrm{mmol}, 222.7 \mathrm{mg}$, $35 \%), \mathrm{R}_{\mathrm{f}}=0.3$ (hexane/EA = 4:1). ${ }^{1} \mathbf{H}$ NMR $\left(400 \mathrm{MHz}, \mathrm{CDCl}_{3}\right): \delta 8.05(\mathrm{~d}, J=8.7 \mathrm{~Hz}, 2 \mathrm{H}), 7.65(\mathrm{~d}, J=8.3 \mathrm{~Hz}$, 2H), $7.35(\mathrm{~d}, J=8.7 \mathrm{~Hz}, 2 \mathrm{H}), 7.23(\mathrm{~d}, J=8.1 \mathrm{~Hz}, 2 \mathrm{H}), 4.32(\mathrm{~s}, 2 \mathrm{H}), 2.41(\mathrm{~s}, 3 \mathrm{H}) \mathrm{ppm} .{ }^{13} \mathbf{C} \mathbf{N M R}(101 \mathrm{MHz}$, $\left.\mathrm{CDCl}_{3}\right): \delta 147.4,145.3,141.9,141.9,130.0,129.9,127.1,123.9,39.4,21.7 \mathrm{ppm}$. The spectral data are in full accordance with the literature report. ${ }^{7}$<smiles>Cc1ccc(S(=O)(=O)C/C=C/c2ccccc2)cc1</smiles>

1aa: S-cinnamyl 4-methylbenzenesulfonothioate. Prepared according to the general procedure C. Corresponding alkyl bromide $(473.0 \mathrm{mg}, 2.4 \mathrm{mmol})$ was converted to 1aa as a yellow solid $(0.85 \mathrm{mmol}, 259.0$ $\mathrm{mg}, 43 \%), \mathrm{R}_{\mathrm{f}}=0.3$ (hexane/EA = 10:1). ${ }^{\mathbf{1}} \mathbf{H} \mathbf{~ N M R}\left(400 \mathrm{MHz}, \mathrm{CDCl}_{3}\right): \delta 7.80(\mathrm{~d}, J=8.4 \mathrm{~Hz}, 2 \mathrm{H}), 7.31-7.23$ $(\mathrm{m}, 5 \mathrm{H}), 7.22-7.18(\mathrm{~m}, 2 \mathrm{H}), 6.46(\mathrm{~d}, J=15.7 \mathrm{~Hz}, 1 \mathrm{H}), 5.98-5.91(\mathrm{~m}, 1 \mathrm{H}), 3.87(\mathrm{dd}, J=7.4,1.1 \mathrm{~Hz}, 2 \mathrm{H})$, $2.37(\mathrm{~s}, 3 \mathrm{H}) \mathrm{ppm} .{ }^{13} \mathrm{C}$ NMR $\left(126 \mathrm{MHz}, \mathrm{CDCl}_{3}\right): \delta 144.8,142.4,135.9,134.9,129.8,128.6,128.2,127.2,126.5$, 121.4, 39.0, 21.6 ppm. HRMS m/z (ESI): calcd. for $\mathrm{C}_{16} \mathrm{H}_{16} \mathrm{O}_{2} \mathrm{~S}_{2} \mathrm{Na}$ [M+Na] $]^{+}$: 327.0489; found: 327.0484 .<smiles>Cc1ccc(S(=O)(=O)CC#Cc2ccccc2)cc1</smiles>

1ab: $S$-(3-phenylprop-2-yn-1-yl) 4-methylbenzenesulfonothioate. Prepared according to the general procedure C. Corresponding alkyl bromide $(959.9 \mathrm{mg}, 4.9 \mathrm{mmol})$ was converted to $\mathbf{1 a b}$ as a yellow solid (3.0 mmol, $918.7 \mathrm{mg}, 76 \%), \mathrm{R}_{\mathrm{f}}=0.3$ (hexane/EA = 10:1). ${ }^{1} \mathbf{H} \mathbf{N M R}\left(400 \mathrm{MHz}, \mathrm{CDCl}_{3}\right): \delta 7.88(\mathrm{~d}, J=8.3 \mathrm{~Hz}, 2 \mathrm{H})$, $7.34-7.24(\mathrm{~m}, 5 \mathrm{H}), 7.19(\mathrm{~d}, J=6.8 \mathrm{~Hz}, 2 \mathrm{H}), 4.06(\mathrm{~s}, 2 \mathrm{H}), 2.33(\mathrm{~s}, 3 \mathrm{H}) \mathrm{ppm} .{ }^{\mathbf{1 3}} \mathbf{C} \mathbf{N M R}\left(101 \mathrm{MHz}, \mathrm{CDCl}_{3}\right): \delta$ 
145.1, 142.0, 131.7, 129.8, 128.6, 128.2, 127.4, 122.1, 85.1, 81.7, 25.6, 21.6 ppm. HRMS m/z (ESI): calcd. for $\mathrm{C}_{16} \mathrm{H}_{14} \mathrm{O}_{2} \mathrm{~S}_{2} \mathrm{Na}[\mathrm{M}+\mathrm{Na}]^{+}: 325.0327$; found: 325.0325 .

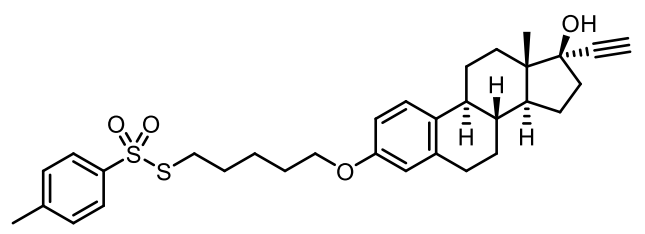

1ad: $\quad S$-(5-(((8R,9S,13S,14S,17R)-17-ethynyl-17-hydroxy-13-methyl-7,8,9,11,12,13,14,15,16,17-decahydro6H-cyclopenta[a]phenanthren-3-yl)oxy)pentyl) 4-methylbenzenesulfonothioate. Prepared according to the general procedure C. Corresponding alkyl bromide $(583.6 \mathrm{mg}, 1.3 \mathrm{mmol}$ ) was converted to 1ad as a yellow oil (0.83 mmol, $461.2 \mathrm{mg}, 64 \%$, containing small amount of solvent DCM and EA), $\mathrm{R}_{\mathrm{f}}=0.1$ (hexane/EA = 4:1). ${ }^{1} \mathbf{H}$ NMR $\left(400 \mathrm{MHz}, \mathrm{CDCl}_{3}\right): \delta 7.80(\mathrm{~d}, J=8.2 \mathrm{~Hz}, 2 \mathrm{H}), 7.32(\mathrm{~d}, J=8.1 \mathrm{~Hz}, 2 \mathrm{H}), 7.18(\mathrm{~d}, J=8.6 \mathrm{~Hz}, 1 \mathrm{H}), 6.66$ $(\mathrm{d}, J=8.6 \mathrm{~Hz}, 1 \mathrm{H}), 6.59(\mathrm{~s}, 1 \mathrm{H}), 3.85(\mathrm{t}, J=6.1 \mathrm{~Hz}, 2 \mathrm{H}), 3.00(\mathrm{t}, J=7.3 \mathrm{~Hz}, 2 \mathrm{H}), 2.87-2.78(\mathrm{~m}, 2 \mathrm{H}), 2.62(\mathrm{~s}$, $1 \mathrm{H}), 2.42(\mathrm{~s}, 3 \mathrm{H}), 2.37-2.28(\mathrm{~m}, 2 \mathrm{H}), 2.25-2.16(\mathrm{~m}, 1 \mathrm{H}), 2.03-1.60(\mathrm{~m}, 11 \mathrm{H}), 1.51-1.30(\mathrm{~m}, 6 \mathrm{H}), 0.88(\mathrm{~s}$, $3 \mathrm{H}) \mathrm{ppm} .{ }^{13} \mathbf{C} \mathbf{N M R}\left(101 \mathrm{MHz}, \mathrm{CDCl}_{3}\right): \delta 156.6,144.6,141.8,137.7,132.3,129.7,126.8,126.2,114.2,111.8$, 87.6, 79.5, 73.8, 67.1, 49.3, 46.9, 43.4, 39.3, 38.8, 35.7, 32.6, 29.6, 28.4, 28.2, 27.1, 26.3, 25.0, 22.7, $21.5,12.6$ ppm. HRMS m/z (ESI): calcd. for $\mathrm{C}_{32} \mathrm{H}_{40} \mathrm{O}_{4} \mathrm{~S}_{2} \mathrm{Na}[\mathrm{M}+\mathrm{Na}]^{+}$: 575.2260 ; found: 575.2258.<smiles>Cc1ccc([As](F)(F)(F)(F)(F)c2ccc(C)cc2)cc1</smiles>

2a: $\boldsymbol{p}$-tolyl(trifluoromethyl)sulfane. Prepared according to the general procedure A. Reaction was run using 1a (111.2 mg, $0.4 \mathrm{mmol}), \mathrm{TMSCF}_{3}(180 \mu \mathrm{L}, 1.2 \mathrm{mmol})$ and NaOAc $(98.4 \mathrm{mg}, 1.2 \mathrm{mmol})$ in DMSO (0.4 mL). The product was purified by flash column chromatography on silica gel (hexane) and obtained as a colorless oil (0.30 mmol, $57.3 \mathrm{mg}, 75 \%), \mathrm{R}_{\mathrm{f}}=0.8$ (hexane). ${ }^{1} \mathbf{H} \mathbf{N M R}\left(400 \mathrm{MHz}, \mathrm{CDCl}_{3}\right): \delta 7.54(\mathrm{~d}, J=8.1 \mathrm{~Hz}, 2 \mathrm{H}), 7.23$ $(\mathrm{d}, J=8.0 \mathrm{~Hz}, 2 \mathrm{H}), 2.40(\mathrm{~s}, 3 \mathrm{H}) \mathrm{ppm} .{ }^{13} \mathbf{C} \mathbf{N M R}\left(126 \mathrm{MHz}, \mathrm{CDCl}_{3}\right): \delta 141.5,136.5,130.5,129.8\left(\mathrm{q}, J_{C-F}=\right.$ $307.9 \mathrm{~Hz}), 121.0\left(\mathrm{q}, J_{C-F}=2.0 \mathrm{~Hz}\right), 21.4 \mathrm{ppm} .{ }^{19} \mathbf{F}$ NMR $\left(471 \mathrm{MHz}, \mathrm{CDCl}_{3}\right): \delta-44.46(\mathrm{~s}, 3 \mathrm{~F}) \mathrm{ppm}$. The spectral data are in full accordance with the literature report. ${ }^{8}$<smiles>COc1ccc(S(F)(F)(F)(F)F)cc1</smiles>

2b: (4-methoxyphenyl)(trifluoromethyl)sulfane. Prepared according to the general procedure A. Reaction was run using $1 \mathbf{b}(62.1 \mathrm{mg}, 0.2 \mathrm{mmol}), \mathrm{TMSCF}_{3}(90 \mu \mathrm{L}, 0.6 \mathrm{mmol})$ and $\mathrm{NaOAc}(49.2 \mathrm{mg}, 0.6 \mathrm{mmol})$ in DMSO (0.2 $\mathrm{mL})$. The product was purified by flash column chromatography on silica gel (hexane) and obtained as a colorless oil (0.19 mmol, $38.9 \mathrm{mg}, 93 \%), \mathrm{R}_{\mathrm{f}}=0.8$ (hexane/EA = 10:1). ${ }^{1} \mathbf{H} \mathbf{N M R}\left(500 \mathrm{MHz}, \mathrm{CDCl}_{3}\right): \delta 7.58(\mathrm{~d}$, $J=8.8 \mathrm{~Hz}, 2 \mathrm{H}), 6.93(\mathrm{~d}, J=8.8 \mathrm{~Hz}, 2 \mathrm{H}), 3.84(\mathrm{~s}, 3 \mathrm{H}) \mathrm{ppm} .{ }^{13} \mathbf{C} \mathbf{N M R}\left(126 \mathrm{MHz}, \mathrm{CDCl}_{3}\right): \delta 162.0,138.4,129.7$ $\left(\mathrm{q}, J_{C-F}=308.1 \mathrm{~Hz}\right), 115.1,115.0\left(\mathrm{q}, J_{C-F}=2.0 \mathrm{~Hz}\right), 55.5 \mathrm{ppm} .{ }^{19} \mathbf{F}$ NMR $\left(471 \mathrm{MHz}, \mathrm{CDCl}_{3}\right): \delta-45.17(\mathrm{~s}, 3 \mathrm{~F})$ ppm. The spectral data are in full accordance with the literature report. ${ }^{8}$

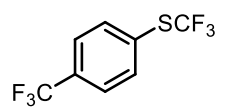

2c: (trifluoromethyl)(4-(trifluoromethyl)phenyl)sulfane. Prepared according to the general procedure A. Reaction was run using 1c $(77.3 \mathrm{mg}, 0.2 \mathrm{mmol}), \mathrm{TMSCF}_{3}(90 \mu \mathrm{L}, 0.6 \mathrm{mmol})$ and $\mathrm{NaOAc}(49.2 \mathrm{mg}, 0.6 \mathrm{mmol})$ in DMSO $(0.2 \mathrm{~mL})$. The product was purified by flash column chromatography on silica gel (hexane) and obtained as a colorless oil (0.09 mmol, $21.4 \mathrm{mg}, 43 \%), \mathrm{R}_{\mathrm{f}}=0.7$ (hexane). ${ }^{1} \mathbf{H}$ NMR (400 MHz, $\left.\mathrm{CDCl}_{3}\right): \delta 7.79$ $(\mathrm{d}, J=8.0 \mathrm{~Hz}, 2 \mathrm{H}), 7.69(\mathrm{~d}, J=8.0 \mathrm{~Hz}, 2 \mathrm{H}) \mathrm{ppm} .{ }^{13} \mathbf{C} \mathbf{N M R}\left(101 \mathrm{MHz}, \mathrm{CDCl}_{3}\right): \delta 136.3,132.9\left(\mathrm{q}, J_{C-F}=32.9\right.$ $\mathrm{Hz}), 129.3\left(\mathrm{q}, J_{C-F}=309.7 \mathrm{~Hz}\right), 129.1\left(\mathrm{~d}, J_{C-F}=2.5 \mathrm{~Hz}\right), 126.5\left(\mathrm{q}, J_{C-F}=3.7 \mathrm{~Hz}\right), 123.6\left(\mathrm{q}, J_{C-F}=273.8 \mathrm{~Hz}\right)$ ppm. ${ }^{19}$ F NMR $\left(377 \mathrm{MHz}, \mathrm{CDCl}_{3}\right): \delta-43.25$ (s, 3F), -64.42 (s, 3F) ppm. HRMS m/z (APCI): calcd. for $\mathrm{C}_{8} \mathrm{H}_{3} \mathrm{~F}_{6} \mathrm{~S}[\mathrm{M}-\mathrm{H}]^{-}: 244.9865$; found: 244.9861.

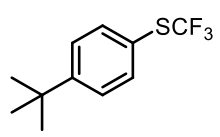

2d: (4-(tert-butyl)phenyl)(trifluoromethyl)sulfane. Prepared according to the general procedure A. Reaction was run using 1 d $(72.5 \mathrm{mg}, 0.2 \mathrm{mmol}), \mathrm{TMSCF}_{3}(90 \mu \mathrm{L}, 0.6 \mathrm{mmol})$ and $\mathrm{NaOAc}(49.2 \mathrm{mg}, 0.6 \mathrm{mmol})$ in DMSO $(0.2 \mathrm{~mL})$. The product was purified by flash column chromatography on silica gel (hexane) and obtained as a colorless oil $(0.13 \mathrm{mmol}, 30.5 \mathrm{mg}, 65 \%), \mathrm{R}_{\mathrm{f}}=0.7$ (hexane). ${ }^{1} \mathbf{H} \mathbf{N M R}\left(400 \mathrm{MHz}, \mathrm{CDCl}_{3}\right): \delta 7.58(\mathrm{~d}, J=8.3 \mathrm{~Hz}$, $2 \mathrm{H}), 7.44(\mathrm{~d}, J=8.4 \mathrm{~Hz}, 2 \mathrm{H}), 1.34(\mathrm{~s}, 9 \mathrm{H}) \mathrm{ppm} .{ }^{13} \mathbf{C} \mathbf{N M R}\left(101 \mathrm{MHz}, \mathrm{CDCl}_{3}\right): \delta 154.5,136.3,129.8\left(\mathrm{q}, J_{C-F}=\right.$ 
$307.9 \mathrm{~Hz}), 126.7,121.0\left(\mathrm{q}, J_{C-F}=2.0 \mathrm{~Hz}\right), 35.0,31.3 \mathrm{ppm} .{ }^{19} \mathbf{F}$ NMR $\left(377 \mathrm{MHz}, \mathrm{CDCl}_{3}\right): \delta-44.20(\mathrm{~s}, 3 \mathrm{~F}) \mathrm{ppm}$. The spectral data are in full accordance with the literature report. ${ }^{9}$<smiles>N#Cc1ccc([Se]C(F)(F)F)cc1</smiles>

2e: 4-((trifluoromethyl)thio)benzonitrile. Prepared according to the general procedure A. Reaction was run using 1e $(60.1 \mathrm{mg}, 0.2 \mathrm{mmol}), \mathrm{TMSCF}_{3}(90 \mu \mathrm{L}, 0.6 \mathrm{mmol})$ and $\mathrm{NaOAc}(49.2 \mathrm{mg}, 0.6 \mathrm{mmol})$ in DMSO (0.2 $\mathrm{mL})$. The product was purified by flash column chromatography on silica gel (hexane) and obtained as a white solid (0.13 mmol, $25.3 \mathrm{mg}, 63 \%), \mathrm{R}_{\mathrm{f}}=0.3$ (hexane). ${ }^{1} \mathbf{H}$ NMR $\left(400 \mathrm{MHz}, \mathrm{CDCl}_{3}\right): \delta 7.76(\mathrm{~d}, J=8.4 \mathrm{~Hz}, 2 \mathrm{H})$, $7.71(\mathrm{~d}, J=8.4 \mathrm{~Hz}, 2 \mathrm{H}) \mathrm{ppm} .{ }^{13} \mathbf{C} \mathbf{N M R}\left(101 \mathrm{MHz}, \mathrm{CDCl}_{3}\right): \delta 136.1,133.0,130.6,129.1\left(\mathrm{q}, J_{C-F}=308.8 \mathrm{~Hz}\right)$, 117.7, $114.7 \mathrm{ppm} .{ }^{19} \mathbf{F}$ NMR $\left(377 \mathrm{MHz}, \mathrm{CDCl}_{3}\right): \delta-42.75(\mathrm{~s}, 3 \mathrm{~F}) \mathrm{ppm}$. The spectral data are in full accordance with the literature report. ${ }^{10}$

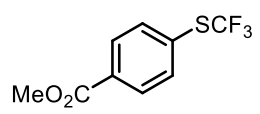

2f: methyl 4-((trifluoromethyl)thio)benzoate. Prepared according to the general procedure A. Reaction was run using 1 f $(73.3 \mathrm{mg}, 0.2 \mathrm{mmol}), \mathrm{TMSCF}_{3}(90 \mu \mathrm{L}, 0.6 \mathrm{mmol})$ and $\mathrm{NaOAc}(49.2 \mathrm{mg}, 0.6 \mathrm{mmol})$ in DMSO (0.2 $\mathrm{mL}$ ). The product was purified by flash column chromatography on silica gel (hexane) and obtained as a colorless oil $(0.17 \mathrm{mmol}, 40.9 \mathrm{mg}, 86 \%), \mathrm{R}_{\mathrm{f}}=0.4$ (hexane). ${ }^{1} \mathbf{H} \mathbf{N M R}\left(400 \mathrm{MHz}, \mathrm{CDCl}_{3}\right): \delta 8.07(\mathrm{~d}, J=8.4 \mathrm{~Hz}$, $2 \mathrm{H}), 7.71(\mathrm{~d}, J=8.3 \mathrm{~Hz}, 2 \mathrm{H}), 3.94(\mathrm{~s}, 3 \mathrm{H}) \mathrm{ppm} .{ }^{13} \mathrm{C}$ NMR $\left(101 \mathrm{MHz}, \mathrm{CDCl}_{3}\right): \delta 166.2,135.7,132.3,130.5$, $130.0\left(\mathrm{q}, J_{C-F}=2.1 \mathrm{~Hz}\right), 129.4\left(\mathrm{q}, J_{C-F}=308.3 \mathrm{~Hz}\right), 52.6 \mathrm{ppm} .{ }^{19} \mathbf{F}$ NMR $\left(377 \mathrm{MHz}, \mathrm{CDCl}_{3}\right): \delta-43.04(\mathrm{~s}, 3 \mathrm{~F})$ ppm. The spectral data are in full accordance with the literature report. ${ }^{10}$<smiles>CSc1ccc(Cl)cc1</smiles>

2g: (4-chlorophenyl)(trifluoromethyl)sulfane. Prepared according to the general procedure A. Reaction was run using $1 \mathrm{~g}(63.8 \mathrm{mg}, 0.2 \mathrm{mmol}), \mathrm{TMSCF}_{3}(90 \mu \mathrm{L}, 0.6 \mathrm{mmol})$ and $\mathrm{NaOAc}(49.2 \mathrm{mg}, 0.6 \mathrm{mmol})$ in DMSO (0.2 $\mathrm{mL})$. The product was purified by flash column chromatography on silica gel (hexane) and obtained as a colorless oil $(0.15 \mathrm{mmol}, 32.9 \mathrm{mg}, 77 \%), \mathrm{R}_{\mathrm{f}}=0.8$ (hexane). ${ }^{1} \mathbf{H} \mathbf{N M R}\left(400 \mathrm{MHz}, \mathrm{CDCl}_{3}\right): \delta 7.59(\mathrm{~d}, J=8.5$ $\mathrm{Hz}, 2 \mathrm{H}), 7.41(\mathrm{~d}, J=8.5 \mathrm{~Hz}, 2 \mathrm{H}) \mathrm{ppm} .{ }^{13} \mathbf{C} \mathbf{N M R}\left(101 \mathrm{MHz}, \mathrm{CDCl}_{3}\right): \delta 137.8,137.7,129.9,129.4\left(\mathrm{q}, J_{C-F}=\right.$ $308.1 \mathrm{~Hz}), 122.9\left(\mathrm{q}, J_{C-F}=2.0 \mathrm{~Hz}\right) \mathrm{ppm} .{ }^{19} \mathbf{F}$ NMR $\left(377 \mathrm{MHz}, \mathrm{CDCl}_{3}\right): \delta-43.97(\mathrm{~s}, 3 \mathrm{~F}) \mathrm{ppm}$. The spectral data are in full accordance with the literature report. ${ }^{8}$<smiles>F[S-](F)c1ccc(Br)cc1</smiles>

2h: (4-bromophenyl)(trifluoromethyl)sulfane. Prepared according to the general procedure A. Reaction was run using $\mathbf{1 h}(81.6 \mathrm{mg}, 0.2 \mathrm{mmol}), \mathrm{TMSCF}_{3}(90 \mu \mathrm{L}, 0.6 \mathrm{mmol})$ and NaOAc $(49.2 \mathrm{mg}, 0.6 \mathrm{mmol})$ in DMSO (0.2 $\mathrm{mL})$. The product was purified by flash column chromatography on silica gel (hexane) and obtained as a colorless oil $(0.15 \mathrm{mmol}, 38.1 \mathrm{mg}, 74 \%), \mathrm{R}_{\mathrm{f}}=0.7$ (hexane). ${ }^{1} \mathbf{H} \mathbf{N M R}\left(400 \mathrm{MHz}, \mathrm{CDCl}_{3}\right): \delta 7.57(\mathrm{~d}, J=8.6 \mathrm{~Hz}$, $2 \mathrm{H}), 7.52(\mathrm{~d}, J=8.5 \mathrm{~Hz}, 2 \mathrm{H}) \mathrm{ppm} .{ }^{13} \mathbf{C} \mathbf{N M R}\left(101 \mathrm{MHz}, \mathrm{CDCl}_{3}\right): \delta 137.9,132.9,129.3\left(\mathrm{q}, J_{C-F}=308.2 \mathrm{~Hz}\right)$, $126.1,123.5\left(\mathrm{q}, J_{C-F}=2.1 \mathrm{~Hz}\right) \mathrm{ppm} .{ }^{19} \mathbf{F}$ NMR $\left(377 \mathrm{MHz}, \mathrm{CDCl}_{3}\right): \delta-44.02(\mathrm{~s}, 3 \mathrm{~F}) \mathrm{ppm}$. The spectral data are in full accordance with the literature report. ${ }^{8}$<smiles></smiles>

2i: (3-bromophenyl)(trifluoromethyl)sulfane. Prepared according to the general procedure A. Reaction was run using $1 \mathbf{i}(81.6 \mathrm{mg}, 0.2 \mathrm{mmol}), \mathrm{TMSCF}_{3}(90 \mu \mathrm{L}, 0.6 \mathrm{mmol})$ and $\mathrm{NaOAc}(49.2 \mathrm{mg}, 0.6 \mathrm{mmol})$ in DMSO $(0.2$ $\mathrm{mL})$. The product was purified by flash column chromatography on silica gel (hexane) and obtained as a colorless oil (0.12 mmol, $31.3 \mathrm{mg}, 61 \%), \mathrm{R}_{\mathrm{f}}=0.7$ (hexane). ${ }^{1} \mathbf{H} \mathbf{N M R}\left(400 \mathrm{MHz}, \mathrm{CDCl}_{3}\right): \delta 7.82(\mathrm{~s}, 1 \mathrm{H}), 7.69-$ $7.55(\mathrm{~m}, 2 \mathrm{H}), 7.31(\mathrm{td}, J=7.9 \mathrm{~Hz}, J=3.0 \mathrm{~Hz}, 1 \mathrm{H}) \mathrm{ppm} .{ }^{13} \mathrm{C}$ NMR $\left(101 \mathrm{MHz}, \mathrm{CDCl}_{3}\right): \delta 138.8,134.9,134.1$, $130.9,129.4\left(\mathrm{q}, J_{C-F}=308.3 \mathrm{~Hz}\right), 126.4,123.0 \mathrm{ppm} .{ }^{19} \mathbf{F}$ NMR $\left(377 \mathrm{MHz}, \mathrm{CDCl}_{3}\right): \delta-43.58(\mathrm{~s}, 3 \mathrm{~F}) \mathrm{ppm}$. The spectral data are in full accordance with the literature report. ${ }^{11}$<smiles>[AsH3-][AsH2]c1cccs1</smiles>

2j: 2-(trifluoromethylthio)thiophene. Prepared according to the general procedure A. Reaction was run using $\mathbf{1 j}(52.5 \mathrm{mg}, 0.2 \mathrm{mmol}), \mathrm{TMSCF}_{3}(90 \mu \mathrm{L}, 0.6 \mathrm{mmol})$ and $\mathrm{NaOAc}(49.2 \mathrm{mg}, 0.6 \mathrm{mmol})$ in DMSO $(0.2 \mathrm{~mL})$. The 
product was purified by flash column chromatography on silica gel (hexane) and obtained as a colorless oil (0.07 mmol, $12.7 \mathrm{mg}, 34 \%), \mathrm{R}_{\mathrm{f}}=0.8$ (hexane). ${ }^{1} \mathbf{H} \mathbf{N M R}\left(400 \mathrm{MHz}, \mathrm{CDCl}_{3}\right): \delta 7.62$ (dd, $J=5.4 \mathrm{~Hz}, J=1.2$ $\mathrm{Hz}, 1 \mathrm{H}), 7.42(\mathrm{dd}, J=3.6 \mathrm{~Hz}, J=1.2 \mathrm{~Hz}, 1 \mathrm{H}), 7.12(\mathrm{dd}, J=5.4 \mathrm{~Hz}, J=3.7 \mathrm{~Hz}, 1 \mathrm{H}) \mathrm{ppm} .{ }^{13} \mathbf{C ~ N M R}(126 \mathrm{MHz}$, $\left.\mathrm{CDCl}_{3}\right): \delta 139.6,134.3,128.5\left(\mathrm{q}, J_{C-F}=310.3 \mathrm{~Hz}\right), 128.3,121.0\left(\mathrm{~d}, J_{C-F}=2.4 \mathrm{~Hz}\right) \mathrm{ppm} .{ }^{19} \mathbf{F} \mathbf{~ N M R}(471 \mathrm{MHz}$, $\left.\mathrm{CDCl}_{3}\right): \delta-46.60(\mathrm{~s}, 3 \mathrm{~F}) \mathrm{ppm}$. The spectral data are in full accordance with the literature report. ${ }^{12}$<smiles>FC(F)(F)c1cccc2ccccc12</smiles>

2k: naphthalen-1-yl(trifluoromethyl)sulfane. Prepared according to the general procedure A. Reaction was run using $1 \mathbf{k}(70.1 \mathrm{mg}, 0.2 \mathrm{mmol}), \mathrm{TMSCF}_{3}(90 \mu \mathrm{L}, 0.6 \mathrm{mmol})$ and NaOAc (49.2 mg, $\left.0.6 \mathrm{mmol}\right)$ in DMSO (0.2 $\mathrm{mL})$. The product was purified by flash column chromatography on silica gel (hexane) and obtained as a white solid (0.12 mmol, $26.8 \mathrm{mg}, 59 \%), \mathrm{R}_{\mathrm{f}}=0.7$ (hexane). ${ }^{1} \mathbf{H}$ NMR $\left(400 \mathrm{MHz}, \mathrm{CDCl}_{3}\right): \delta 8.56(\mathrm{~d}, J=8.5 \mathrm{~Hz}, 1 \mathrm{H})$, $8.02(\mathrm{~d}, J=8.3 \mathrm{~Hz}, 1 \mathrm{H}), 7.98(\mathrm{~d}, J=7.1 \mathrm{~Hz}, 1 \mathrm{H}), 7.91(\mathrm{~d}, J=8.1 \mathrm{~Hz}, 1 \mathrm{H}), 7.67(\mathrm{t}, J=7.3 \mathrm{~Hz}, 1 \mathrm{H}), 7.59(\mathrm{t}, J=$ $7.5 \mathrm{~Hz}, 1 \mathrm{H}), 7.52(\mathrm{t}, J=7.7 \mathrm{~Hz}, 1 \mathrm{H}) \mathrm{ppm} .{ }^{13} \mathbf{C}$ NMR $\left(101 \mathrm{MHz}, \mathrm{CDCl}_{3}\right): \delta 137.9,135.5,134.4,132.5,129.8(\mathrm{q}$, $\left.J_{C-F}=313.4 \mathrm{~Hz}\right), 128.7,127.8,126.9,126.0,125.7,121.7$ ppm. ${ }^{19} \mathbf{F}$ NMR $\left(377 \mathrm{MHz}, \mathrm{CDCl}_{3}\right): \delta-43.41(\mathrm{~s}, 3 \mathrm{~F})$ ppm. The spectral data are in full accordance with the literature report. ${ }^{13}$<smiles>[Se]c1ccc2c(c1)CCO2</smiles>

2l: 5-((trifluoromethyl)thio)-2,3-dihydrobenzofuran. Prepared according to the general procedure A. Reaction was run using 11 (66.9 mg, $0.2 \mathrm{mmol}), \mathrm{TMSCF}_{3}(90 \mu \mathrm{L}, 0.6 \mathrm{mmol})$ and NaOAc $(49.2 \mathrm{mg}, 0.6 \mathrm{mmol})$ in DMSO $(0.2 \mathrm{~mL})$. The product was purified by flash column chromatography on silica gel (hexane) and obtained as a colorless oil $(0.11 \mathrm{mmol}, 24.4 \mathrm{mg}, 55 \%), \mathrm{R}_{\mathrm{f}}=0.3$ (hexane). ${ }^{1} \mathbf{H}$ NMR (400 MHz, $\left.\mathrm{CDCl}_{3}\right): \delta 7.46$ $(\mathrm{s}, 1 \mathrm{H}), 7.41(\mathrm{~d}, J=8.3 \mathrm{~Hz}, 1 \mathrm{H}), 6.80(\mathrm{~d}, J=8.3 \mathrm{~Hz}, 1 \mathrm{H}), 4.64(\mathrm{t}, J=8.8 \mathrm{~Hz}, 2 \mathrm{H}), 3.25(\mathrm{t}, J=8.8 \mathrm{~Hz}, 2 \mathrm{H}) \mathrm{ppm}$. ${ }^{13}$ C NMR (101 MHz, $\left.\mathrm{CDCl}_{3}\right): \delta 162.8,137.6,133.6,129.8\left(\mathrm{q}, J_{C-F}=309.9 \mathrm{~Hz}\right), 129.0,114.3\left(\mathrm{~d}, J_{C-F}=2.2 \mathrm{~Hz}\right)$, 110.4, 72.1, 29.4 ppm. ${ }^{19} \mathbf{F}$ NMR $\left(377 \mathrm{MHz}, \mathrm{CDCl}_{3}\right): \delta-45.38$ (s, 3F) ppm. The spectral data are in full accordance with the literature report. ${ }^{9}$<smiles>O=C(OCCCCCC(F)(F)F)c1ccccc1</smiles>

2m: 5-((trifluoromethyl)thio)pentyl benzoate. Prepared according to the general procedure A. Reaction was run using $1 \mathrm{~m}(75,7 \mathrm{mg}, 0.2 \mathrm{mmol}), \mathrm{TMSCF}_{3}(90 \mu \mathrm{L}, 0.6 \mathrm{mmol})$ and $\mathrm{NaOAc}(49.2 \mathrm{mg}, 0.6 \mathrm{mmol})$ in DMSO $(0.2 \mathrm{~mL})$. The product was purified by flash column chromatography on silica gel (hexane $/ \mathrm{EA}=50: 1)$ and obtained as a colorless oil $(0.19 \mathrm{mmol}, 55.1 \mathrm{mg}, 94 \%), \mathrm{R}_{\mathrm{f}}=0.3$ (hexane/EA = 40:1). ${ }^{1} \mathbf{H}$ NMR $(400 \mathrm{MHz}$, $\left.\mathrm{CDCl}_{3}\right): \delta 8.04(\mathrm{~d}, J=7.1 \mathrm{~Hz}, 2 \mathrm{H}), 7.56(\mathrm{t}, J=7.4 \mathrm{~Hz}, 1 \mathrm{H}), 7.44(\mathrm{t}, J=7.7 \mathrm{~Hz}, 2 \mathrm{H}), 4.33(\mathrm{t}, J=6.5 \mathrm{~Hz}, 2 \mathrm{H})$, $2.91(\mathrm{t}, J=7.4 \mathrm{~Hz}, 2 \mathrm{H}), 1.85-1.73(\mathrm{~m}, 4 \mathrm{H}), 1.63-1.52(\mathrm{~m}, 2 \mathrm{H}) \mathrm{ppm} .{ }^{13} \mathbf{C} \mathbf{N M R}\left(101 \mathrm{MHz}, \mathrm{CDCl}_{3}\right): \delta 166.7$, 133.0, $131.2\left(\mathrm{q}, J_{C-F}=305.7 \mathrm{~Hz}\right), 130.4,129.6,128.5,64.6,29.8\left(\mathrm{~d}, J_{C-F}=1.9 \mathrm{~Hz}\right), 29.2,28.2,25.1 \mathrm{ppm} .{ }^{19} \mathbf{F}$ NMR $\left(377 \mathrm{MHz}, \mathrm{CDCl}_{3}\right): \delta-42.24(\mathrm{~s}, 3 \mathrm{~F}) \mathrm{ppm}$. The spectral data are in full accordance with the literature report. $^{14}$<smiles>O=C(OCCC(F)(F)F)c1ccccc1</smiles>

2n: 2-((trifluoromethyl)thio)ethyl benzoate. Prepared according to the general procedure A. Reaction was run using 1n $(67.3 \mathrm{mg}, 0.2 \mathrm{mmol}), \mathrm{TMSCF}_{3}(90 \mu \mathrm{L}, 0.6 \mathrm{mmol})$ and $\mathrm{NaOAc}(49.2 \mathrm{mg}, 0.6 \mathrm{mmol})$ in DMSO $(0.2$ $\mathrm{mL}$ ). The product was purified by flash column chromatography on silica gel (hexane/EA $=50: 1$ ) and obtained as a colorless oil $(0.17 \mathrm{mmol}, 43.5 \mathrm{mg}, 87 \%), \mathrm{R}_{\mathrm{f}}=0.3$ (hexane/EA $\left.=40: 1\right)$. ${ }^{1} \mathbf{H} \mathbf{~ N M R}\left(400 \mathrm{MHz}, \mathrm{CDCl}_{3}\right): \delta$ $8.05(\mathrm{~d}, J=7.2 \mathrm{~Hz}, 2 \mathrm{H}), 7.58(\mathrm{t}, J=7.4 \mathrm{~Hz}, 1 \mathrm{H}), 7.46(\mathrm{t}, J=7.7 \mathrm{~Hz}, 2 \mathrm{H}), 4.56(\mathrm{t}, J=6.5 \mathrm{~Hz}, 2 \mathrm{H}), 3.27(\mathrm{t}, J=$ $6.5 \mathrm{~Hz}, 2 \mathrm{H}) \mathrm{ppm} .{ }^{13} \mathrm{C}$ NMR $\left(101 \mathrm{MHz}, \mathrm{CDCl}_{3}\right): \delta 166.2,133.4,130.9$ (q, $\left.J_{C-F}=306.3 \mathrm{~Hz}\right), 129.8,129.6,128.7$, 63.0, $28.7\left(\mathrm{q}, J_{C-F}=2.1 \mathrm{~Hz}\right)$ ppm. ${ }^{19} \mathbf{F}$ NMR $\left(377 \mathrm{MHz}, \mathrm{CDCl}_{3}\right): \delta-42.18(\mathrm{~s}, 3 \mathrm{~F}) \mathrm{ppm}$. HRMS m/z (ESI): calcd. for $\mathrm{C}_{10} \mathrm{H}_{9} \mathrm{~F}_{3} \mathrm{O}_{2} \mathrm{SNa}[\mathrm{M}+\mathrm{Na}]^{+}$: 273.0168; found: 273.0170 .

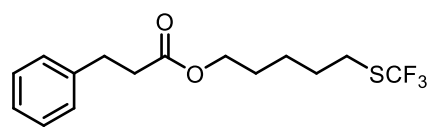

2o: 5-((trifluoromethyl)thio)pentyl 3-phenylpropanoate. Prepared according to the general procedure A. Reaction was run using $10(81.3 \mathrm{mg}, 0.2 \mathrm{mmol}), \mathrm{TMSCF}_{3}(90 \mu \mathrm{L}, 0.6 \mathrm{mmol})$ and $\mathrm{NaOAc}(49.2 \mathrm{mg}, 0.6 \mathrm{mmol})$ 
in DMSO $(0.2 \mathrm{~mL})$. The product was purified by flash column chromatography on silica gel (hexane/EA = 40:1) and obtained as a colorless oil $(0.19 \mathrm{mmol}, 61.7 \mathrm{mg}, 96 \%), \mathrm{R}_{\mathrm{f}}=0.2$ (hexane/EA $\left.=40: 1\right) .{ }^{1} \mathbf{H}$ NMR $(400 \mathrm{MHz}$, $\left.\mathrm{CDCl}_{3}\right): \delta 7.34-7.27(\mathrm{~m}, 2 \mathrm{H}), 7.25-7.18(\mathrm{~m}, 3 \mathrm{H}), 4.08(\mathrm{t}, J=6.5 \mathrm{~Hz}, 2 \mathrm{H}), 2.97(\mathrm{t}, J=7.7 \mathrm{~Hz}, 2 \mathrm{H}), 2.87(\mathrm{t}, J$ $=7.4 \mathrm{~Hz}, 2 \mathrm{H}), 2.65(\mathrm{t}, J=7.8 \mathrm{~Hz}, 2 \mathrm{H}), 1.76-1.58(\mathrm{~m}, 4 \mathrm{H}), 1.47-1.36(\mathrm{~m}, 2 \mathrm{H}) \mathrm{ppm} .{ }^{13} \mathbf{C}$ NMR $(126 \mathrm{MHz}$, $\left.\mathrm{CDCl}_{3}\right): \delta 173.0,140.5,131.2\left(\mathrm{q}, J_{C-F}=305.7 \mathrm{~Hz}\right), 128.5,128.3,126.3,64.1,35.9,31.0,29.7\left(\mathrm{~d}, J_{C-F}=2.0 \mathrm{~Hz}\right)$, 29.1, 28.1, 24.9 ppm. ${ }^{19} \mathbf{F}$ NMR (377 MHz, $\mathrm{CDCl}_{3}$ ): $\delta-42.24$ (s, 3F) ppm. HRMS m/z (ESI): calcd. for $\mathrm{C}_{15} \mathrm{H}_{19} \mathrm{~F}_{3} \mathrm{O}_{2} \mathrm{SNa}[\mathrm{M}+\mathrm{Na}]^{+}:$343.0951; found: 343.0954 .<smiles>O=C(c1ccccc1)N1CCC(C[Se])CC1</smiles>

2p: tert-butyl 4-(((trifluoromethyl)thio)methyl)piperidine-1-carboxylate. Prepared according to the general procedure A. Reaction was run using $1 \mathbf{p}(77.1 \mathrm{mg}, 0.2 \mathrm{mmol}), \mathrm{TMSCF}_{3}(90 \mu \mathrm{L}, 0.6 \mathrm{mmol})$ and $\mathrm{NaOAc}(49.2$ $\mathrm{mg}, 0.6 \mathrm{mmol})$ in DMSO $(0.2 \mathrm{~mL})$. The product was purified by flash column chromatography on silica gel (hexane/EA = 40:1) and obtained as a colorless oil $(0.19 \mathrm{mmol}, 55.9 \mathrm{mg}, 93 \%), \mathrm{R}_{\mathrm{f}}=0.2$ (hexane/EA = 40:1). ${ }^{1} \mathbf{H}$ NMR $\left(400 \mathrm{MHz}, \mathrm{CDCl}_{3}\right): \delta 4.11(\mathrm{~d}, J=7.3 \mathrm{~Hz}, 2 \mathrm{H}), 2.77(\mathrm{~d}, J=7.0 \mathrm{~Hz}, 2 \mathrm{H}), 2.66(\mathrm{t}, J=12.2 \mathrm{~Hz}, 2 \mathrm{H}), 1.79$ $(\mathrm{d}, J=12.9 \mathrm{~Hz}, 2 \mathrm{H}), 1.75-1.63(\mathrm{~m}, 1 \mathrm{H}), 1.43(\mathrm{~s}, 9 \mathrm{H}), 1.11(\mathrm{qd}, J=12.4 \mathrm{~Hz}, J=4.1 \mathrm{~Hz}, 2 \mathrm{H}) \mathrm{ppm} .{ }^{13} \mathbf{C}$ NMR $\left(126 \mathrm{MHz}, \mathrm{CDCl}_{3}\right): \delta 154.8,131.1\left(\mathrm{q}, J_{C-F}=305.6 \mathrm{~Hz}\right), 79.6,43.6,36.2,35.9\left(\mathrm{~d}, J_{C-F}=1.6 \mathrm{~Hz}\right), 31.3,28.5 \mathrm{ppm}$. ${ }^{19}$ F NMR $\left(377 \mathrm{MHz}, \mathrm{CDCl}_{3}\right): \delta-42.23$ (s, 3F) ppm. HRMS m/z (ESI): calcd. for $\mathrm{C}_{12} \mathrm{H}_{20} \mathrm{~F}_{3} \mathrm{NO}_{2} \mathrm{SNa}[\mathrm{M}+\mathrm{Na}]^{+}$: 322.1059; found: 322.1060 .

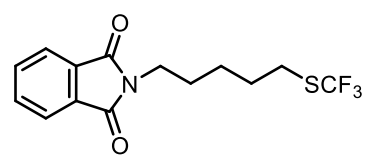

2q: 2-(5-((trifluoromethyl)thio)pentyl)isoindoline-1,3-dione. Prepared according to the general procedure A. Reaction was run using 1q ( $80.7 \mathrm{mg}, 0.2 \mathrm{mmol}), \mathrm{TMSCF}_{3}(90 \mu \mathrm{L}, 0.6 \mathrm{mmol})$ and $\mathrm{NaOAc}(49.2 \mathrm{mg}, 0.6 \mathrm{mmol})$ in DMSO $(0.2 \mathrm{~mL})$. The product was purified by flash column chromatography on silica gel (hexane/EA $=20: 1$ ) and obtained as a colorless oil $(0.15 \mathrm{mmol}, 49.1 \mathrm{mg}, 77 \%), \mathrm{R}_{\mathrm{f}}=0.2$ (hexane/EA $\left.=20: 1\right) .{ }^{1} \mathbf{H}$ NMR $(400 \mathrm{MHz}$, $\left.\mathrm{CDCl}_{3}\right): \delta 7.83-7.81(\mathrm{~m}, 2 \mathrm{H}), 7.71-7.69(\mathrm{~m}, 2 \mathrm{H}), 3.67(\mathrm{t}, J=7.2 \mathrm{~Hz}, 2 \mathrm{H}), 2.85(\mathrm{t}, J=7.4 \mathrm{~Hz}, 2 \mathrm{H}), 1.76-$ $1.66(\mathrm{~m}, 4 \mathrm{H}), 1.48-1.40(\mathrm{~m}, 2 \mathrm{H}) \mathrm{ppm} .{ }^{13} \mathbf{C} \mathbf{~ N M R}\left(101 \mathrm{MHz}, \mathrm{CDCl}_{3}\right): \delta 168.5,134.0,132.2,131.2\left(\mathrm{q}, J_{C-F}=\right.$ $305.7 \mathrm{~Hz}), 123.3,37.7,29.7\left(\mathrm{~d}, J_{C-F}=1.9 \mathrm{~Hz}\right), 29.1,28.0,25.8 \mathrm{ppm} .{ }^{19} \mathbf{F}$ NMR $\left(377 \mathrm{MHz}, \mathrm{CDCl}_{3}\right): \delta-42.30(\mathrm{~s}$, $3 \mathrm{~F}) \mathrm{ppm}$. The spectral data are in full accordance with the literature report. ${ }^{15}$<smiles>CC(C)(C)CCCCCO[Si](c1ccccc1)(c1ccccc1)C(C)(C)C</smiles>

2r: tert-butyldiphenyl((5-((trifluoromethyl)thio)pentyl)oxy)silane. Prepared according to the general procedure A. Reaction was run using $1 \mathbf{r}(102.6 \mathrm{mg}, 0.2 \mathrm{mmol}), \mathrm{TMSCF}_{3}(90 \mu \mathrm{L}, 0.6 \mathrm{mmol})$ and $\mathrm{NaOAc}^{2}(49.2$ $\mathrm{mg}, 0.6 \mathrm{mmol})$ in DMSO $(0.2 \mathrm{~mL})$. The product was purified by flash column chromatography on silica gel (hexane) and obtained as a colorless oil $(0.10 \mathrm{mmol}, 44.3 \mathrm{mg}, 52 \%), \mathrm{R}_{\mathrm{f}}=0.3$ (hexane). ${ }^{1} \mathbf{H}$ NMR (400 MHz, $\left.\mathrm{CDCl}_{3}\right): \delta 7.69(\mathrm{dd}, J=7.8 \mathrm{~Hz}, J=1.6 \mathrm{~Hz}, 4 \mathrm{H}), 7.47-7.37(\mathrm{~m}, 6 \mathrm{H}), 3.68(\mathrm{t}, J=6.1 \mathrm{~Hz}, 2 \mathrm{H}), 2.87(\mathrm{t}, J=7.4$ $\mathrm{Hz}, 2 \mathrm{H}), 1.75-1.64(\mathrm{~m}, 2 \mathrm{H}), 1.64-1.55(\mathrm{~m}, 2 \mathrm{H}), 1.54-1.46(\mathrm{~m}, 2 \mathrm{H}), 1.07(\mathrm{~s}, 9 \mathrm{H}) \mathrm{ppm} .{ }^{13} \mathbf{C}$ NMR $(101 \mathrm{MHz}$, $\left.\mathrm{CDCl}_{3}\right): \delta 135.7,134.1,131.3\left(\mathrm{q}, J_{C-F}=305.6 \mathrm{~Hz}\right), 129.7,127.8,63.6,31.9,30.0\left(\mathrm{~d}, J_{C-F}=1.9 \mathrm{~Hz}\right), 29.3,27.0$, 25.0, $19.3 \mathrm{ppm} .{ }^{19} \mathbf{F}$ NMR (377 $\mathrm{MHz}, \mathrm{CDCl}_{3}$ ): $\delta-42.26$ (s, 3F) ppm. HRMS m/z (APCI): calcd. for $\mathrm{C}_{22} \mathrm{H}_{30} \mathrm{~F}_{3} \mathrm{SOSi}[\mathrm{M}+\mathrm{H}]^{+}$: 427.1733 ; found: 427.1732 .<smiles>[AsH3]CCCc1ccccc1</smiles>

2s: (3-phenylpropyl)(trifluoromethyl)sulfane. Prepared according to the general procedure A. Reaction was run using $1 \mathrm{~s}(61.2 \mathrm{mg}, 0.2 \mathrm{mmol})$, $\mathrm{TMSCF}_{3}(90 \mu \mathrm{L}, 0.6 \mathrm{mmol})$ and $\mathrm{NaOAc}(49.2 \mathrm{mg}, 0.6 \mathrm{mmol})$ in DMSO (0.2 $\mathrm{mL}$ ). The product was purified by flash column chromatography on silica gel (hexane) and obtained as a colorless oil ( $0.16 \mathrm{mmol}, 34.5 \mathrm{mg}, 78 \%), \mathrm{R}_{\mathrm{f}}=0.6$ (hexane). ${ }^{1} \mathbf{H} \mathbf{~ N M R}\left(500 \mathrm{MHz}, \mathrm{CDCl}_{3}\right): \delta 7.33(\mathrm{t}, J=7.5 \mathrm{~Hz}$, $2 \mathrm{H}), 7.28-7.24(\mathrm{~m}, 1 \mathrm{H}), 7.22(\mathrm{t}, J=7.2 \mathrm{~Hz}, 2 \mathrm{H}), 2.91(\mathrm{t}, J=7.3 \mathrm{~Hz}, 2 \mathrm{H}), 2.77(\mathrm{t}, J=7.5 \mathrm{~Hz}, 2 \mathrm{H}), 2.06(\mathrm{qn}, J=$ $7.4 \mathrm{~Hz}, 2 \mathrm{H}) \mathrm{ppm} .{ }^{13} \mathbf{C}$ NMR $\left(126 \mathrm{MHz}, \mathrm{CDCl}_{3}\right): \delta 140.6,131.2\left(\mathrm{q}, J_{C-F}=305.8 \mathrm{~Hz}\right), 128.7,128.6,126.4,34.4$, $31.1,29.3\left(\mathrm{~d}, J_{C-F}=1.9 \mathrm{~Hz}\right) \mathrm{ppm} .{ }^{19} \mathbf{F}$ NMR $\left(471 \mathrm{MHz}, \mathrm{CDCl}_{3}\right): \delta-42.22(\mathrm{~s}, 3 \mathrm{~F}) \mathrm{ppm}$. The spectral data are in full accordance with the literature report. ${ }^{16}$ 


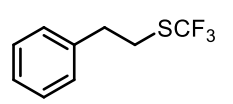

2t: phenethyl(trifluoromethyl)sulfane. Prepared according to the general procedure A. Reaction was run using 1t $(58.5 \mathrm{mg}, 0.2 \mathrm{mmol}), \mathrm{TMSCF}_{3}(90 \mu \mathrm{L}, 0.6 \mathrm{mmol})$ and NaOAc $(49.2 \mathrm{mg}, 0.6 \mathrm{mmol})$ in DMSO $(0.2 \mathrm{~mL})$. The product was purified by flash column chromatography on silica gel (hexane) and obtained as a colorless oil (0.14 mmol, $28.0 \mathrm{mg}, 68 \%), \mathrm{R}_{\mathrm{f}}=0.6$ (hexane). ${ }^{1} \mathbf{H}$ NMR $\left(400 \mathrm{MHz}, \mathrm{CDCl}_{3}\right): \delta 7.35-7.32(\mathrm{~m}, 2 \mathrm{H}), 7.28-7.26$ $(\mathrm{m}, 1 \mathrm{H}), 7.24-7.20(\mathrm{~m}, 2 \mathrm{H}), 3.14(\mathrm{t}, J=7.6 \mathrm{~Hz}, 2 \mathrm{H}), 3.00(\mathrm{t}, J=7.6 \mathrm{~Hz}, 2 \mathrm{H}) \mathrm{ppm} .{ }^{\mathbf{1 3}} \mathbf{C} \mathbf{~ N M R}(101 \mathrm{MHz}$, $\left.\mathrm{CDCl}_{3}\right): \delta 139.0,131.3\left(\mathrm{q}, J_{C-F}=305.9 \mathrm{~Hz}\right), 128.8,128.6,127.0,36.1,31.3\left(\mathrm{~d}, J_{C-F}=2.0 \mathrm{~Hz}\right) \mathrm{ppm} .{ }^{19} \mathbf{F}$ NMR $\left(377 \mathrm{MHz}, \mathrm{CDCl}_{3}\right): \delta-42.15(\mathrm{~s}, 3 \mathrm{~F}) \mathrm{ppm}$. The spectral data are in full accordance with the literature report. ${ }^{17}$<smiles>CCC(c1ccccc1)C(F)(F)F</smiles>

2u: (1-phenylpropyl)(trifluoromethyl)sulfane. Prepared according to the general procedure A. Reaction was run using $1 \mathbf{u}(61.3 \mathrm{mg}, 0.2 \mathrm{mmol}), \mathrm{TMSCF}_{3}(90 \mu \mathrm{L}, 0.6 \mathrm{mmol})$ and $\mathrm{NaOAc}(49.2 \mathrm{mg}, 0.6 \mathrm{mmol})$ in DMSO (0.2 $\mathrm{mL})$. The product was purified by flash column chromatography on silica gel (hexane) and obtained as a colorless oil $(0.14 \mathrm{mmol}, 30.0 \mathrm{mg}, 68 \%), \mathrm{R}_{\mathrm{f}}=0.6$ (hexane). ${ }^{1} \mathbf{H} \mathbf{~ N M R}\left(400 \mathrm{MHz}, \mathrm{CDCl}_{3}\right): \delta 7.38-7.34(\mathrm{~m}$, $2 \mathrm{H}), 7.32-7.28(\mathrm{~m}, 3 \mathrm{H}), 4.22(\mathrm{dd}, J=8.8 \mathrm{~Hz}, J=6.4 \mathrm{~Hz}, 1 \mathrm{H}), 2.12-1.93(\mathrm{~m}, 2 \mathrm{H}), 0.93(\mathrm{t}, J=7.4 \mathrm{~Hz}, 3 \mathrm{H})$ ppm. ${ }^{13} \mathrm{C}$ NMR $\left(101 \mathrm{MHz}, \mathrm{CDCl}_{3}\right): \delta 140.6,130.7\left(\mathrm{q}, J_{C-F}=307.0 \mathrm{~Hz}\right), 128.8,127.9,127.6,51.4\left(\mathrm{~d}, J_{C-F}=1.4\right.$ $\mathrm{Hz}$ ), 30.0, 12.0 ppm. ${ }^{19} \mathbf{F}$ NMR $\left(377 \mathrm{MHz}, \mathrm{CDCl}_{3}\right): \delta-40.91$ (s, 3F) ppm. HRMS m/z (APCI): calcd. for $\mathrm{C}_{10} \mathrm{H}_{10} \mathrm{~F}_{3} \mathrm{~S}[\mathrm{M}-\mathrm{H}]^{-}: 219.0461$; found: 219.0461.<smiles>COc1ccc(CC(F)(F)F)cc1</smiles>

2v: (4-methoxybenzyl)(trifluoromethyl)sulfane. Prepared according to the general procedure A. Reaction was run using $1 \mathbf{v}(61.7 \mathrm{mg}, 0.2 \mathrm{mmol}), \mathrm{TMSCF}_{3}(90 \mu \mathrm{L}, 0.6 \mathrm{mmol})$ and $\mathrm{NaOAc}(49.2 \mathrm{mg}, 0.6 \mathrm{mmol})$ in DMSO $(0.2$ $\mathrm{mL}$ ). The product was purified by flash column chromatography on silica gel (hexane) and obtained as a colorless oil ( $0.16 \mathrm{mmol}, 36.4 \mathrm{mg}, 82 \%), \mathrm{R}_{\mathrm{f}}=0.3$ (hexane). ${ }^{1} \mathbf{H} \mathbf{N M R}\left(400 \mathrm{MHz}, \mathrm{CDCl}_{3}\right): \delta 7.27(\mathrm{~d}, J=8.6 \mathrm{~Hz}$, $2 \mathrm{H}), 6.88(\mathrm{~d}, J=8.6 \mathrm{~Hz}, 2 \mathrm{H}), 4.10(\mathrm{~s}, 2 \mathrm{H}), 3.81(\mathrm{~s}, 3 \mathrm{H}) \mathrm{ppm} .{ }^{13} \mathbf{C}$ NMR $\left(101 \mathrm{MHz}, \mathrm{CDCl}_{3}\right): \delta 159.5,130.8(\mathrm{q}$, $\left.J_{C-F}=307.0 \mathrm{~Hz}\right), 130.3,126.9,114.4,55.4,33.9\left(\mathrm{q}, J_{C-F}=2.3 \mathrm{~Hz}\right) \mathrm{ppm} .{ }^{19} \mathbf{F}$ NMR $\left(377 \mathrm{MHz}, \mathrm{CDCl}_{3}\right): \delta-42.64$ $(\mathrm{s}, 3 \mathrm{~F}) \mathrm{ppm}$. The spectral data are in full accordance with the literature report. ${ }^{17}$<smiles>CC(C)(C)c1ccc(CC(F)(F)F)cc1</smiles>

2w: (4-(tert-butyl)benzyl)(trifluoromethyl)sulfane. Prepared according to the general procedure A. Reaction was run using $\mathbf{1 w}(66.9 \mathrm{mg}, 0.2 \mathrm{mmol}), \mathrm{TMSCF}_{3}(90 \mu \mathrm{L}, 0.6 \mathrm{mmol})$ and $\mathrm{NaOAc}(49.2 \mathrm{mg}, 0.6 \mathrm{mmol})$ in DMSO $(0.2 \mathrm{~mL})$. The product was purified by flash column chromatography on silica gel (hexane) and obtained as a colorless oil $(0.12 \mathrm{mmol}, 30.5 \mathrm{mg}, 61 \%), \mathrm{R}_{\mathrm{f}}=0.6$ (hexane). ${ }^{1} \mathbf{H} \mathbf{N M R}\left(400 \mathrm{MHz}, \mathrm{CDCl}_{3}\right): \delta 7.40(\mathrm{~d}, J=8.2 \mathrm{~Hz}$, $2 \mathrm{H}), 7.30(\mathrm{~d}, J=8.1 \mathrm{~Hz}, 2 \mathrm{H}), 4.13(\mathrm{~s}, 2 \mathrm{H}), 1.34(\mathrm{~s}, 9 \mathrm{H}) \mathrm{ppm} .{ }^{13} \mathbf{C}$ NMR $\left(101 \mathrm{MHz}, \mathrm{CDCl}_{3}\right): \delta 151.2,131.9$, $130.8\left(\mathrm{q}, J_{C-F}=306.8 \mathrm{~Hz}\right), 128.8,125.9,34.7,34.0\left(\mathrm{q}, J_{C-F}=2.3 \mathrm{~Hz}\right), 31.4 \mathrm{ppm} .{ }^{19} \mathbf{F} \mathbf{N M R}\left(377 \mathrm{MHz}, \mathrm{CDCl}_{3}\right): \delta$ $-42.75(\mathrm{~s}, 3 \mathrm{~F}) \mathrm{ppm}$. The spectral data are in full accordance with the literature report. ${ }^{18}$<smiles>CC(C)(F)Cc1ccc(Cl)cc1</smiles>

2x: (4-chlorobenzyl)(trifluoromethyl)sulfane. Prepared according to the general procedure A. Reaction was run using 1x (62.6 mg, $0.2 \mathrm{mmol}), \mathrm{TMSCF}_{3}(90 \mu \mathrm{L}, 0.6 \mathrm{mmol})$ and NaOAc $(49.2 \mathrm{mg}, 0.6 \mathrm{mmol})$ in DMSO (0.2 $\mathrm{mL})$. The product was purified by flash column chromatography on silica gel (hexane) and obtained as a colorless oil (0.13 mmol, $29.5 \mathrm{mg}, 65 \%), \mathrm{R}_{\mathrm{f}}=0.6$ (hexane). ${ }^{1} \mathbf{H} \mathbf{N M R}\left(400 \mathrm{MHz}, \mathrm{CDCl}_{3}\right): \delta 7.32(\mathrm{~d}, J=8.5 \mathrm{~Hz}$, $2 \mathrm{H}), 7.28(\mathrm{~d}, J=8.6 \mathrm{~Hz}, 2 \mathrm{H}), 4.08(\mathrm{~s}, 2 \mathrm{H}) \mathrm{ppm} .{ }^{13} \mathrm{C}$ NMR $\left(101 \mathrm{MHz}, \mathrm{CDCl}_{3}\right): \delta 134.2,133.8,130.6\left(\mathrm{q}, J_{C-F}=\right.$ $307.0 \mathrm{~Hz}), 130.4,129.2,33.7\left(\mathrm{q}, J_{C-F}=2.4 \mathrm{~Hz}\right)$ ppm. ${ }^{19} \mathbf{F}$ NMR $\left(377 \mathrm{MHz}, \mathrm{CDCl}_{3}\right): \delta-42.55(\mathrm{~s}, 3 \mathrm{~F}) \mathrm{ppm}$. The spectral data are in full accordance with the literature report. ${ }^{17}$<smiles>F[C-](F)Cc1ccc(Br)cc1</smiles>

2y: (4-bromobenzyl)(trifluoromethyl)sulfane. Prepared according to the general procedure A. Reaction was run using $1 \mathbf{y}(71.5 \mathrm{mg}, 0.2 \mathrm{mmol}), \mathrm{TMSCF}_{3}(90 \mu \mathrm{L}, 0.6 \mathrm{mmol})$ and $\mathrm{NaOAc}(49.2 \mathrm{mg}, 0.6 \mathrm{mmol})$ in DMSO (0.2 $\mathrm{mL})$. The product was purified by flash column chromatography on silica gel (hexane) and obtained as a colorless oil $(0.13 \mathrm{mmol}, 33.9 \mathrm{mg}, 63 \%), \mathrm{R}_{\mathrm{f}}=0.6$ (hexane). ${ }^{1} \mathbf{H} \mathbf{N M R}\left(400 \mathrm{MHz}, \mathrm{CDCl}_{3}\right): \delta 7.48(\mathrm{~d}, J=8.4 \mathrm{~Hz}$, 
$2 \mathrm{H}), 7.22(\mathrm{~d}, J=8.3 \mathrm{~Hz}, 2 \mathrm{H}), 4.06(\mathrm{~s}, 2 \mathrm{H}) \mathrm{ppm} .{ }^{13} \mathrm{C}$ NMR $\left(101 \mathrm{MHz}, \mathrm{CDCl}_{3}\right): \delta 134.3,132.1,130.7,130.6(\mathrm{q}$, $\left.J_{C-F}=308.2 \mathrm{~Hz}\right), 122.2,33.8\left(\mathrm{q}, J_{C-F}=2.4 \mathrm{~Hz}\right) \mathrm{ppm} .{ }^{19} \mathbf{F}$ NMR $\left(377 \mathrm{MHz}, \mathrm{CDCl}_{3}\right): \delta-42.54(\mathrm{~s}, 3 \mathrm{~F}) \mathrm{ppm}$. The spectral data are in full accordance with the literature report. ${ }^{16}$<smiles>O=[N+]([O-])c1ccc(CC(F)(F)F)cc1</smiles>

2z: (4-nitrobenzyl)(trifluoromethyl)sulfane. Prepared according to the general procedure A. Reaction was run using $1 \mathbf{z}(64.7 \mathrm{mg}, 0.2 \mathrm{mmol}), \mathrm{TMSCF}_{3}(90 \mu \mathrm{L}, 0.6 \mathrm{mmol})$ and $\mathrm{NaHCO}_{3}(16.8 \mathrm{mg}, 0.2 \mathrm{mmol})$ in DMSO $(0.2$ $\mathrm{mL}$ ). The product was purified by flash column chromatography on silica gel (hexane/EA $=20: 1$ ) and obtained as a colorless oil $(0.08 \mathrm{mmol}, 19.3 \mathrm{mg}, 41 \%), \mathrm{R}_{\mathrm{f}}=0.6$ (hexane/EA $\left.=4: 1\right) .{ }^{1} \mathbf{H} \mathbf{N M R}\left(400 \mathrm{MHz}, \mathrm{CDCl}_{3}\right): \delta 7.39$ $(\mathrm{d}, J=8.1 \mathrm{~Hz}, 2 \mathrm{H}), 7.11(\mathrm{~d}, J=8.0 \mathrm{~Hz}, 2 \mathrm{H}), 2.33(\mathrm{~s}, 2 \mathrm{H}) \mathrm{ppm} .{ }^{13} \mathbf{C}$ NMR $\left(126 \mathrm{MHz}, \mathrm{CDCl}_{3}\right): \delta 147.7,143.1$, $130.4\left(\mathrm{q}, J_{C-F}=308.0 \mathrm{~Hz}\right), 129.9,124.2,33.6\left(\mathrm{q}, J_{C-F}=2.4 \mathrm{~Hz}\right) \mathrm{ppm} .{ }^{19} \mathbf{F} \mathbf{N M R}\left(471 \mathrm{MHz}, \mathrm{CDCl}_{3}\right): \delta-42.38(\mathrm{~s}$, $3 \mathrm{~F}) \mathrm{ppm}$. The spectral data are in full accordance with the literature report. ${ }^{15}$<smiles>FC(F)([Mg])CCC=Cc1ccccc1</smiles>

2aa: cinnamyl(trifluoromethyl)sulfane. Prepared according to the general procedure A. Reaction was run using 1aa $(60.9 \mathrm{mg}, 0.2 \mathrm{mmol}), \mathrm{TMSCF}_{3}(90 \mu \mathrm{L}, 0.6 \mathrm{mmol})$ and $\mathrm{NaHCO}_{3}(16.8 \mathrm{mg}, 0.2 \mathrm{mmol})$ in DMSO (0.2 $\mathrm{mL})$. The product was purified by flash column chromatography on silica gel (hexane) and obtained as a colorless oil $(0.15 \mathrm{mmol}, 33.6 \mathrm{mg}, 77 \%), \mathrm{R}_{\mathrm{f}}=0.6$ (hexane). ${ }^{1} \mathbf{H}$ NMR $\left(400 \mathrm{MHz}, \mathrm{CDCl}_{3}\right): \delta 7.40-7.39(\mathrm{~m}$, $2 \mathrm{H}), 7.37-7.33(\mathrm{~m}, 2 \mathrm{H}), 7.31-7.26(\mathrm{~m}, 1 \mathrm{H}), 6.62(\mathrm{~d}, J=15.7 \mathrm{~Hz}, 1 \mathrm{H}), 6.28-6.21(\mathrm{~m}, 1 \mathrm{H}), 3.74(\mathrm{~d}, J=7.4$ $\mathrm{Hz}, 2 \mathrm{H}) \mathrm{ppm} .{ }^{13} \mathbf{C} \mathbf{N M R}\left(101 \mathrm{MHz}, \mathrm{CDCl}_{3}\right): \delta 136.2,134.5,130.9\left(\mathrm{q}, J_{C-F}=306.9 \mathrm{~Hz}\right), 128.8,128.2,126.6$, $123.1,32.8\left(\mathrm{q}, J_{C-F}=2.3 \mathrm{~Hz}\right) \mathrm{ppm} .{ }^{19} \mathbf{F}$ NMR $\left(471 \mathrm{MHz}, \mathrm{CDCl}_{3}\right): \delta-41.93(\mathrm{~s}, 3 \mathrm{~F}) \mathrm{ppm}$. The spectral data are in full accordance with the literature report. ${ }^{17}$

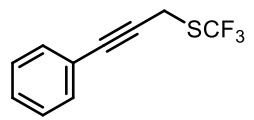

2ab: (3-phenylprop-2-yn-1-yl)(trifluoromethyl)sulfane. Prepared according to the general procedure A. Reaction was run using 1ab $(60.5 \mathrm{mg}, 0.2 \mathrm{mmol}), \mathrm{TMSCF}_{3}(90 \mu \mathrm{L}, 0.6 \mathrm{mmol})$ and $\mathrm{NaHCO}_{3}(16.8 \mathrm{mg}, 0.2$ $\mathrm{mmol})$ in DMSO $(0.2 \mathrm{~mL})$. The product was purified by flash column chromatography on silica gel (hexane) and obtained as a colorless oil $(0.05 \mathrm{mmol}, 11.6 \mathrm{mg}, 27 \%), \mathrm{R}_{\mathrm{f}}=0.5$ (hexane). ${ }^{1} \mathbf{H} \mathbf{~ N M R}\left(400 \mathrm{MHz}, \mathrm{CDCl}_{3}\right): \delta$ $7.47-7.40(\mathrm{~m}, 2 \mathrm{H}), 7.35-7.29(\mathrm{~m}, 3 \mathrm{H}), 3.90(\mathrm{~s}, 2 \mathrm{H}) \mathrm{ppm} .{ }^{13} \mathbf{C} \mathbf{N M R}\left(101 \mathrm{MHz}, \mathrm{CDCl}_{3}\right): \delta 131.9,130.5\left(\mathrm{q}, J_{C-}\right.$ $F=307.5 \mathrm{~Hz}), 128.8,128.4,122.3,84.7,82.3,19.6\left(\mathrm{q}, J_{C-F}=3.4 \mathrm{~Hz}\right)$ ppm. ${ }^{19} \mathbf{F}$ NMR $\left(471 \mathrm{MHz}, \mathrm{CDCl}_{3}\right): \delta-$ $42.89(\mathrm{~s}, 3 \mathrm{~F}) \mathrm{ppm}$. The spectral data are in full accordance with the literature report. ${ }^{16}$<smiles>CCOC(=O)C(CSC(F)(F)F)NC(=O)OCc1ccccc1</smiles>

2ac: ethyl $\boldsymbol{N}$-(tert-butoxycarbonyl)-S-(trifluoromethyl)-L-cysteinate. Prepared according to the general procedure A. Reaction was run using 1 ac $(80.7 \mathrm{mg}, 0.2 \mathrm{mmol}), \mathrm{TMSCF}_{3}(90 \mu \mathrm{L}, 0.6 \mathrm{mmol})$ and $\mathrm{NaHCO}_{3}(16.8$ $\mathrm{mg}, 0.2 \mathrm{mmol})$ in DMSO $(0.2 \mathrm{~mL})$. The product was purified by flash column chromatography on silica gel (hexane/EA = 20:1) and obtained as a colorless oil $(0.09 \mathrm{mmol}, 27.6 \mathrm{mg}, 43 \%), \mathrm{R}_{\mathrm{f}}=0.6$ (hexane/EA = 10:1). ${ }^{1} \mathbf{H}$ NMR $\left(400 \mathrm{MHz}, \mathrm{CDCl}_{3}\right): \delta 5.37(\mathrm{~s}, 1 \mathrm{H}), 4.58(\mathrm{~s}, 1 \mathrm{H}), 4.24(\mathrm{q}, J=7.0 \mathrm{~Hz}, 2 \mathrm{H}), 3.63-3.15(\mathrm{~m}, 2 \mathrm{H}), 1.44(\mathrm{~s}$, $9 \mathrm{H}), 1.30(\mathrm{t}, J=7.0 \mathrm{~Hz}, 3 \mathrm{H}) \mathrm{ppm} .{ }^{13} \mathbf{C} \mathbf{N M R}\left(126 \mathrm{MHz}, \mathrm{CDCl}_{3}\right): \delta 169.7,155.0,130.5\left(\mathrm{q}, J_{C-F}=306.0 \mathrm{~Hz}\right)$, 80.7, 62.4, 53.1, 32.2, 28.3, 14.2 ppm. ${ }^{19} \mathbf{F}$ NMR $\left(471 \mathrm{MHz}, \mathrm{CDCl}_{3}\right): \delta-42.24(\mathrm{~s}, 3 \mathrm{~F}) \mathrm{ppm}$. The spectral data are in full accordance with the literature report. ${ }^{19}$

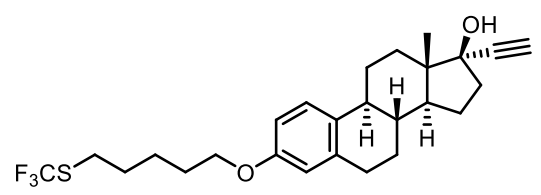

2ad: $\quad(8 R, 9 S, 13 S, 14 S, 17 R)$-17-ethynyl-13-methyl-3-((5-((trifluoromethyl)thio)pentyl)oxy)$\mathbf{7 , 8 , 9 , 1 1 , 1 2 , 1 3 , 1 4 , 1 5 , 1 6 , 1 7 - d e c a h y d r o - 6 H - c y c l o p e n t a [ a ] p h e n a n t h r e n - 1 7 - o l . ~ P r e p a r e d ~ a c c o r d i n g ~ t o ~ t h e ~}$ general procedure A. Reaction was run using 1 ad $(110.6 \mathrm{mg}, 0.2 \mathrm{mmol}), \mathrm{TMSCF}_{3}(90 \mu \mathrm{L}, 0.6 \mathrm{mmol})$ and $\mathrm{NaOAc}(49.2 \mathrm{mg}, 0.6 \mathrm{mmol})$ in DMSO $(0.2 \mathrm{~mL})$. The product was purified by flash column chromatography on silica gel (hexane/EA $=15: 1)$ and obtained as a yellow oil $(0.11 \mathrm{mmol}, 51.6 \mathrm{mg}, 55 \%), \mathrm{R}_{\mathrm{f}}=0.4$ (hexane/EA = 4:1). ${ }^{1} \mathbf{H}$ NMR $\left(400 \mathrm{MHz}, \mathrm{CDCl}_{3}\right): \delta 7.21(\mathrm{~d}, J=8.6 \mathrm{~Hz}, 1 \mathrm{H}), 6.70(\mathrm{~d}, J=8.5 \mathrm{~Hz}, 1 \mathrm{H}), 6.63(\mathrm{~s}, 1 \mathrm{H}), 3.95(\mathrm{t}, J=$ 
$6.1 \mathrm{~Hz}, 2 \mathrm{H}), 2.91(\mathrm{t}, J=7.3 \mathrm{~Hz}, 2 \mathrm{H}), 2.85(\mathrm{~d}, J=4.6 \mathrm{~Hz}, 2 \mathrm{H}), 2.61(\mathrm{~s}, 1 \mathrm{H}), 2.44-2.30(\mathrm{~m}, 2 \mathrm{H}), 2.30-2.19(\mathrm{~m}$, $1 \mathrm{H}), 2.05-1.85(\mathrm{~m}, 4 \mathrm{H}), 1.85-1.69(\mathrm{~m}, 7 \mathrm{H}), 1.64-1.56(\mathrm{~m}, 2 \mathrm{H}), 1.52-1.33(\mathrm{~m}, 4 \mathrm{H}), 0.89(\mathrm{~s}, 3 \mathrm{H}) \mathrm{ppm} .{ }^{13} \mathrm{C}$ NMR $\left(126 \mathrm{MHz}, \mathrm{CDCl}_{3}\right): \delta 156.9,138.0,132.6,131.3\left(\mathrm{q}, J_{C-F}=305.7 \mathrm{~Hz}\right), 126.5,114.5,112.1,87.6,80.0$, 74.1, 67.4, 49.5, 47.2, 43.6, 39.5, 39.0, 32.8, 29.9, 29.8 (d, $\left.J_{C-F}=1.9 \mathrm{~Hz}\right), 29.3,28.8,27.3,26.5,25.3,22.9,12.8$ ppm. ${ }^{19} \mathbf{F}$ NMR (471 MHz, $\mathrm{CDCl}_{3}$ ): $\delta-42.28$ (s, 3F) ppm. HRMS m/z (APCI): calcd. for $\mathrm{C}_{26} \mathrm{H}_{34} \mathrm{~F}_{3} \mathrm{O}_{2} \mathrm{~S}[\mathrm{M}+\mathrm{H}]^{+}$: 467.2226; found: 467.2228 .

SCFF $\mathrm{CF}_{3}$

3a: (4-methoxyphenyl)(pentafluoroethyl)sulfane. Prepared according to the general procedure A. Reaction was run using $\mathbf{1 b}(62.1 \mathrm{mg}, 0.2 \mathrm{mmol}), \mathrm{TMSCF}_{2} \mathrm{CF}_{3}(105 \mu \mathrm{L}, 0.6 \mathrm{mmol})$ and $\mathrm{NaOAc}(49.2 \mathrm{mg}, 0.6 \mathrm{mmol})$ in DMSO $(0.2 \mathrm{~mL})$. The product was purified by flash column chromatography on silica gel (hexane/EA $=50: 1$ ) and obtained as a colorless oil $(0.18 \mathrm{mmol}, 46.9 \mathrm{mg}, 91 \%), \mathrm{R}_{\mathrm{f}}=0.8$ (hexane/EA = 10:1). ${ }^{\mathbf{1}} \mathbf{H} \mathbf{~ N M R}(400 \mathrm{MHz}$, $\left.\mathrm{CDCl}_{3}\right): \delta 7.57(\mathrm{~d}, J=8.8 \mathrm{~Hz}, 2 \mathrm{H}), 6.93(\mathrm{~d}, J=8.9 \mathrm{~Hz}, 2 \mathrm{H}), 3.84(\mathrm{~s}, 3 \mathrm{H}) \mathrm{ppm} .{ }^{13} \mathbf{C} \mathbf{N M R}\left(126 \mathrm{MHz}, \mathrm{CDCl}_{3}\right): \delta$ $162.1,139.1,120.1\left(\mathrm{tq}, J_{C-F}=287.9 \mathrm{~Hz}, J_{C-F}=39.9 \mathrm{~Hz}\right), 118.9\left(\mathrm{qt}, J_{C-F}=286.4 \mathrm{~Hz}, J_{C-F}=37.2 \mathrm{~Hz}\right), 115.1$, $113.2\left(\mathrm{t}, J_{C-F}=3.0 \mathrm{~Hz}\right), 55.51 \mathrm{ppm} .{ }^{19} \mathbf{F} \mathbf{N M R}\left(471 \mathrm{MHz}, \mathrm{CDCl}_{3}\right): \delta-83.61(\mathrm{~s}, 3 \mathrm{~F}),-93.98(\mathrm{~d}, J=2.4 \mathrm{~Hz}, 2 \mathrm{~F})$ $\mathrm{ppm}$. The spectral data are in full accordance with the literature report. ${ }^{20}$<smiles>O=C(OCCCCCC(F)(F)F)c1ccccc1</smiles>

3b: 5-((pentafluoroethyl)thio)pentyl benzoate. Prepared according to the general procedure A. Reaction was run using $1 \mathrm{~m}(75.7 \mathrm{mg}, 0.2 \mathrm{mmol}), \mathrm{TMSCF}_{2} \mathrm{CF}_{3}(105 \mu \mathrm{L}, 0.6 \mathrm{mmol})$ and $\mathrm{NaOAc}(49.2 \mathrm{mg}, 0.6 \mathrm{mmol})$ in DMSO $(0.2 \mathrm{~mL})$. The product was purified by flash column chromatography on silica gel (hexane/EA $=40: 1$ ) and obtained as a colorless oil $(0.19 \mathrm{mmol}, 66.0 \mathrm{mg}, 96 \%), \mathrm{R}_{\mathrm{f}}=0.4$ (hexane/EA = 10:1). ${ }^{1} \mathbf{H} \mathbf{~ N M R}(400 \mathrm{MHz}$, $\left.\mathrm{CDCl}_{3}\right): \delta 8.04(\mathrm{~d}, J=7.1 \mathrm{~Hz}, 2 \mathrm{H}), 7.56(\mathrm{t}, J=7.4 \mathrm{~Hz}, 1 \mathrm{H}), 7.44(\mathrm{t}, J=7.7 \mathrm{~Hz}, 2 \mathrm{H}), 4.33(\mathrm{t}, J=6.5 \mathrm{~Hz}, 2 \mathrm{H})$, $2.95(\mathrm{t}, J=7.4 \mathrm{~Hz}, 2 \mathrm{H}), 1.84-1.74(\mathrm{~m}, 4 \mathrm{H}), 1.62-1.55(\mathrm{~m}, 2 \mathrm{H}) \mathrm{ppm} .{ }^{13} \mathbf{C ~ N M R}\left(126 \mathrm{MHz}, \mathrm{CDCl}_{3}\right): \delta 166.7$, $133.0,130.4,129.6,128.5,121.9\left(\mathrm{tq}, J_{C-F}=287.2 \mathrm{~Hz}, J_{C-F}=40.7 \mathrm{~Hz}\right), 118.7\left(\mathrm{qt}, J_{C-F}=285.8 \mathrm{~Hz}, J_{C-F}=37.1\right.$ $\mathrm{Hz}), 64.6,29.3,28.4\left(\mathrm{t}, J_{C-F}=3.3 \mathrm{~Hz}\right), 28.2,25.2 \mathrm{ppm} .{ }^{19} \mathbf{F} \mathbf{N M R}\left(471 \mathrm{MHz}, \mathrm{CDCl}_{3}\right): \delta-84.68(\mathrm{~s}, 3 \mathrm{~F}),-93.31(\mathrm{~d}$, $J=3.0 \mathrm{~Hz}, 2 \mathrm{~F}) \mathrm{ppm}$. HRMS m/z (ESI): calcd. for $\mathrm{C}_{14} \mathrm{H}_{15} \mathrm{~F}_{5} \mathrm{O}_{2} \mathrm{SNa}[\mathrm{M}+\mathrm{Na}]^{+}: 365.0605$; found: 365.0608 .

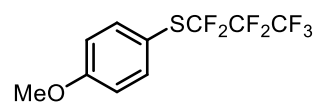

4a: (4-methoxyphenyl)(heptafluoropropyl)sulfane. Prepared according to the general procedure A. Reaction was run using $\mathbf{1 b}(62.1 \mathrm{mg}, 0.2 \mathrm{mmol}), \mathrm{TMSCF}_{2} \mathrm{CF}_{2} \mathrm{CF}_{3}(122 \mu \mathrm{L}, 0.6 \mathrm{mmol})$ and $\mathrm{NaOAc}(49.2 \mathrm{mg}, 0.6 \mathrm{mmol})$ in DMSO $(0.2 \mathrm{~mL})$. The product was purified by flash column chromatography on silica gel (hexane/EA = 50:1) and obtained as a colorless oil $(0.16 \mathrm{mmol}, 49.3 \mathrm{mg}, 80 \%), \mathrm{R}_{\mathrm{f}}=0.7$ (hexane/EA = 10:1). ${ }^{1} \mathbf{H} \mathbf{N M R}(400 \mathrm{MHz}$, $\left.\mathrm{CDCl}_{3}\right): \delta 7.57(\mathrm{~d}, J=8.8 \mathrm{~Hz}, 2 \mathrm{H}), 6.93(\mathrm{~d}, J=8.9 \mathrm{~Hz}, 2 \mathrm{H}), 3.84(\mathrm{~s}, 3 \mathrm{H}) \mathrm{ppm} .{ }^{13} \mathbf{C} \mathbf{N M R}\left(126 \mathrm{MHz}, \mathrm{CDCl}_{3}\right): \delta$ $162.2,139.2,122.2\left(\mathrm{tt}, J_{C-F}=289.1 \mathrm{~Hz}, J_{C-F}=33.2 \mathrm{~Hz}\right), 117.9\left(\mathrm{qt}, J_{C-F}=287.9 \mathrm{~Hz}, J_{C-F}=34.6 \mathrm{~Hz}\right), 115.1,113.0$ $\left(\mathrm{tq}, J_{C-F}=2.8 \mathrm{~Hz}\right), 109.1\left(\mathrm{tq}, J_{C-F}=265.5 \mathrm{~Hz}, J_{C-F}=37.2 \mathrm{~Hz}\right), 55.5 \mathrm{ppm} .{ }^{19} \mathbf{F} \mathbf{N M R}\left(471 \mathrm{MHz}, \mathrm{CDCl}_{3}\right): \delta-81.35$ $(\mathrm{t}, J=9.2 \mathrm{~Hz}, 3 \mathrm{~F}),-89.84--89.91(\mathrm{~m}, 2 \mathrm{~F}),-124.88(\mathrm{t}, J=3.4 \mathrm{~Hz}, 2 \mathrm{~F}) \mathrm{ppm}$. The spectral data are in full accordance with the literature report. ${ }^{20}$

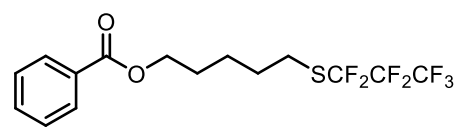

4b: 5-((heptafluoropropyl)thio)pentyl benzoate. Prepared according to the general procedure A. Reaction was run using $1 \mathrm{~m}(75.7 \mathrm{mg}, 0.2 \mathrm{mmol}), \mathrm{TMSCF}_{2} \mathrm{CF}_{2} \mathrm{CF}_{3}(122 \mu \mathrm{L}, 0.6 \mathrm{mmol})$ and $\mathrm{NaOAc}(49.2 \mathrm{mg}, 0.6 \mathrm{mmol})$ in DMSO $(0.2 \mathrm{~mL})$. The product was purified by flash column chromatography on silica gel (hexane/EA $=40: 1$ ) and obtained as a colorless oil $(0.19 \mathrm{mmol}, 73.4 \mathrm{mg}, 94 \%), \mathrm{R}_{\mathrm{f}}=0.4$ (hexane/EA = 10:1). ${ }^{1} \mathbf{H} \mathbf{~ N M R}(400 \mathrm{MHz}$, $\left.\mathrm{CDCl}_{3}\right): \delta 8.04(\mathrm{~d}, J=7.1 \mathrm{~Hz}, 2 \mathrm{H}), 7.56(\mathrm{t}, J=7.4 \mathrm{~Hz}, 1 \mathrm{H}), 7.44(\mathrm{t}, J=7.7 \mathrm{~Hz}, 2 \mathrm{H}), 4.33(\mathrm{t}, J=6.5 \mathrm{~Hz}, 2 \mathrm{H})$, $2.96(\mathrm{t}, J=7.4 \mathrm{~Hz}, 2 \mathrm{H}), 1.85-1.75(\mathrm{~m}, 4 \mathrm{H}), 1.63-1.55(\mathrm{~m}, 2 \mathrm{H})$ ppm. ${ }^{13} \mathbf{C}$ NMR $\left(126 \mathrm{MHz}, \mathrm{CDCl}_{3}\right): \delta 166.7$, $133.0,130.4,129.6,128.5,124.2\left(\mathrm{tt}, J_{C-F}=288.3 \mathrm{~Hz}, J_{C-F}=33.1 \mathrm{~Hz}\right), 117.8\left(\mathrm{qt}, J_{C-F}=287.7 \mathrm{~Hz}, J_{C-F}=34.3\right.$ $\mathrm{Hz}), 108.7\left(\mathrm{tq}, J_{C-F}=265.2 \mathrm{~Hz}, J_{C-F}=37.9 \mathrm{~Hz}\right), 64.6,29.4,28.4\left(\mathrm{t}, J_{C-F}=3.6 \mathrm{~Hz}\right), 28.2,25.2 \mathrm{ppm} .{ }^{19} \mathbf{F}$ NMR $\left(471 \mathrm{MHz}, \mathrm{CDCl}_{3}\right): \delta-81.27(\mathrm{t}, J=9.2 \mathrm{~Hz}, 3 \mathrm{~F}),-89.39--89.47(\mathrm{~m}, 2 \mathrm{~F}),-125.40(\mathrm{t}, J=3.9 \mathrm{~Hz}, 3 \mathrm{~F}) \mathrm{ppm}$. HRMS m/z (ESI): calcd. for $\mathrm{C}_{15} \mathrm{H}_{15} \mathrm{~F}_{7} \mathrm{O}_{2} \mathrm{SNa}[\mathrm{M}+\mathrm{Na}]^{+}$: 415.0573 ; found: 415.0571 . 


\section{References}

1. Zhao, X.; Liu, T.-X.; Zhang, G. Asian J. Org. Chem. 2017, 6, 677-681.

2. (a) Zheng, Y.; Qing, F.-L.; Huang, Y.; Xu, X.-H. Adv. Synth. Catal. 2016, 358, 3477-3481. (b) Yoshida, S.; Sugimura, Y.; Hazama, Y.; Nishiyama, Y.; Yano, T.; Shimizu, S.; Hosoya, T. Chem. Commun. 2015, 51, $16613-16616$.

3. Xiao, X.; Feng, M.; Jiang, X. Angew .Chem., Int. Ed. 2016, 55, 14121-14125.

4. Zhang, Y.; Ji, P.; Hu, W.; Wei, Y.; Huang, H.; Wang, W. Chem. - Eur. J. 2019, 25, 8225-8228.

5. Ali, D.; Hunter, R.; Kaschula, C. H.; De Doncker, S.; Rees-Jones, S. C. M. J. Org. Chem. 2019, 84, 28622869.

6. Li, G.; Gan, Z.; Kong, K.; Dou, X.; Yang, D. Adv. Synth. Catal. 2019, 361, 1808-1814.

7. Musiejuk, M.; Doroszuk, J.; Jędrzejewski, B.; Nieto, G. O.; Navarro, M. M.; Witt, D. Adv. Synth. Catal. 2020, $362,618-626$

8. Harsányi, A.; Dorkó, É.; Csapó, Á.; Bakó, T.; Peltz, C.; Rábai, J. J. Fluorine. Chem. 2011, 132, 1241-1246.

9. Shen, F.; Zheng, H.; Xue, X.-S.; Lu, L.; Shen, Q. Org. Lett. 2019, 21, 6347-6351.

10. Matheis, C.; Wagner, V.; Goossen, L. J. Chem. - Eur. J. 2016, 22, 79-82.

11. Koziakov, D.; Majek, M.; Wangelin, A. J. Eur. J. Org. Chem. 2017, 2017, 6722-6725.

12. Weng, Z.; He, W.; Chen, C.; Lee, R.; Tan, D.; Lai, Z.; Kong, D.; Yuan, Y.; Huang, K.-W. Angew. Chem., Int. Ed. 2013, 52, 1548- 1552 .

13. Horvat, M.; Jereb, M.; Iskra, J. Eur. J. Org. Chem. 2018, 2018, 3837-3843.

14. Wang, F.; Xu, P.; Cong, F.; Tang, P. Chem. Sci. 2018, 9, 8836-8841.

15. Ouyang, Y.; Xu, X.-H.; Qing, F.-L. Angew. Chem., Int. Ed. 2019, 58, 18508-18512.

16. Dix, S.; Jakob, M.; Hopkinson, M. N. Chem. - Eur. J. 2019, 25, 7635-7639.

17. Matheis, C.; Wang, M.; Krause, T.; Goossen, L. J. Synlett 2015, 26, 1628-1632.

18. Liu, J.-B.; Xu, X.-H.; Chen, Z.-H.; Qing, F.-L. Angew. Chem., Int. Ed. 2015, 54, 897-900.

19. Zeng, J.-L.; Chachignon, H.; Ma, J.-A.; Cahard, D. Org. Lett. 2017, 19, 1974-1977.

20. Xiang, J.-X.; Xu, X.-H.; Qing, F.-L. J. Fluorine. Chem. 2017, 203, 110-114. 
Spectra of new compounds:
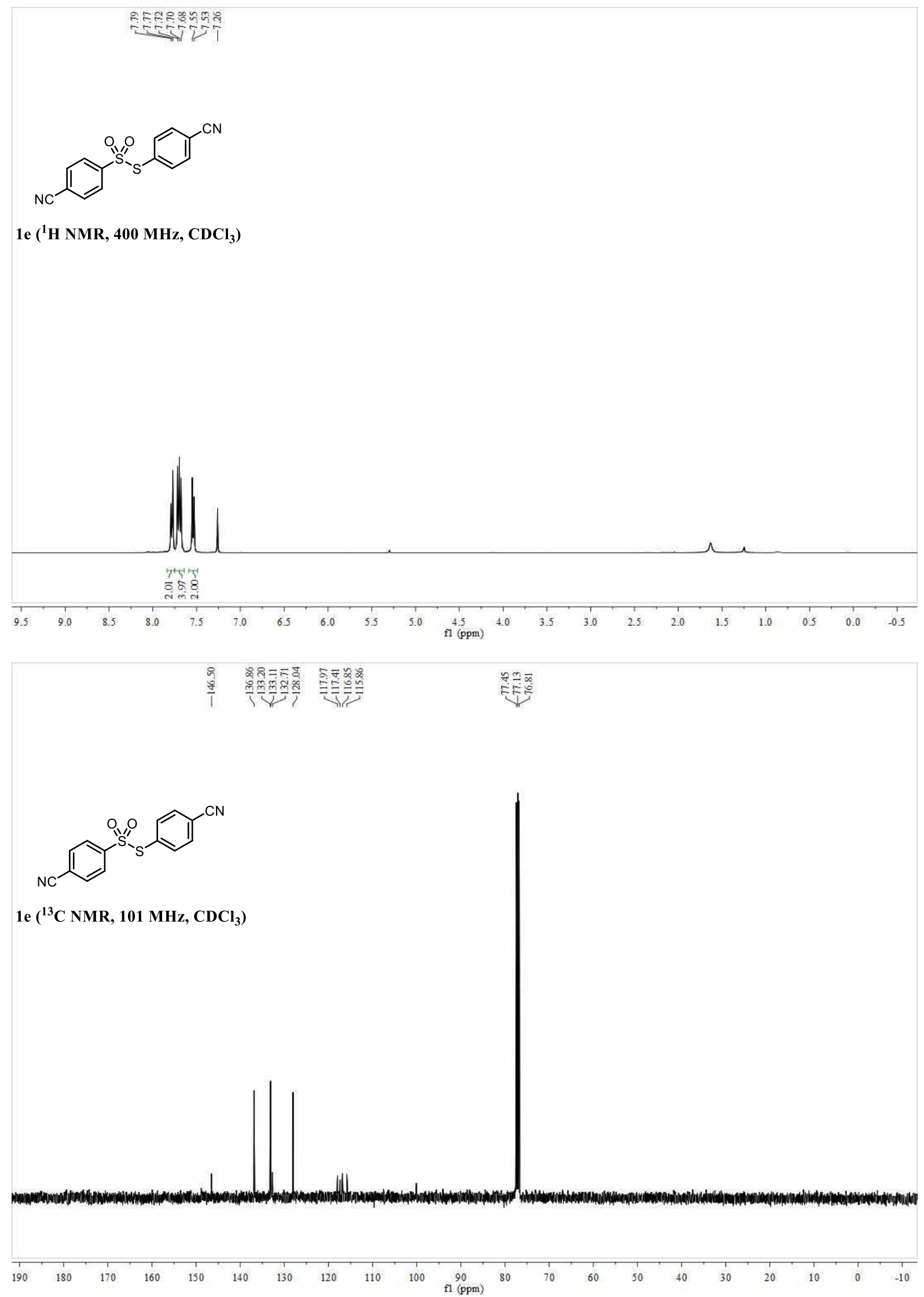

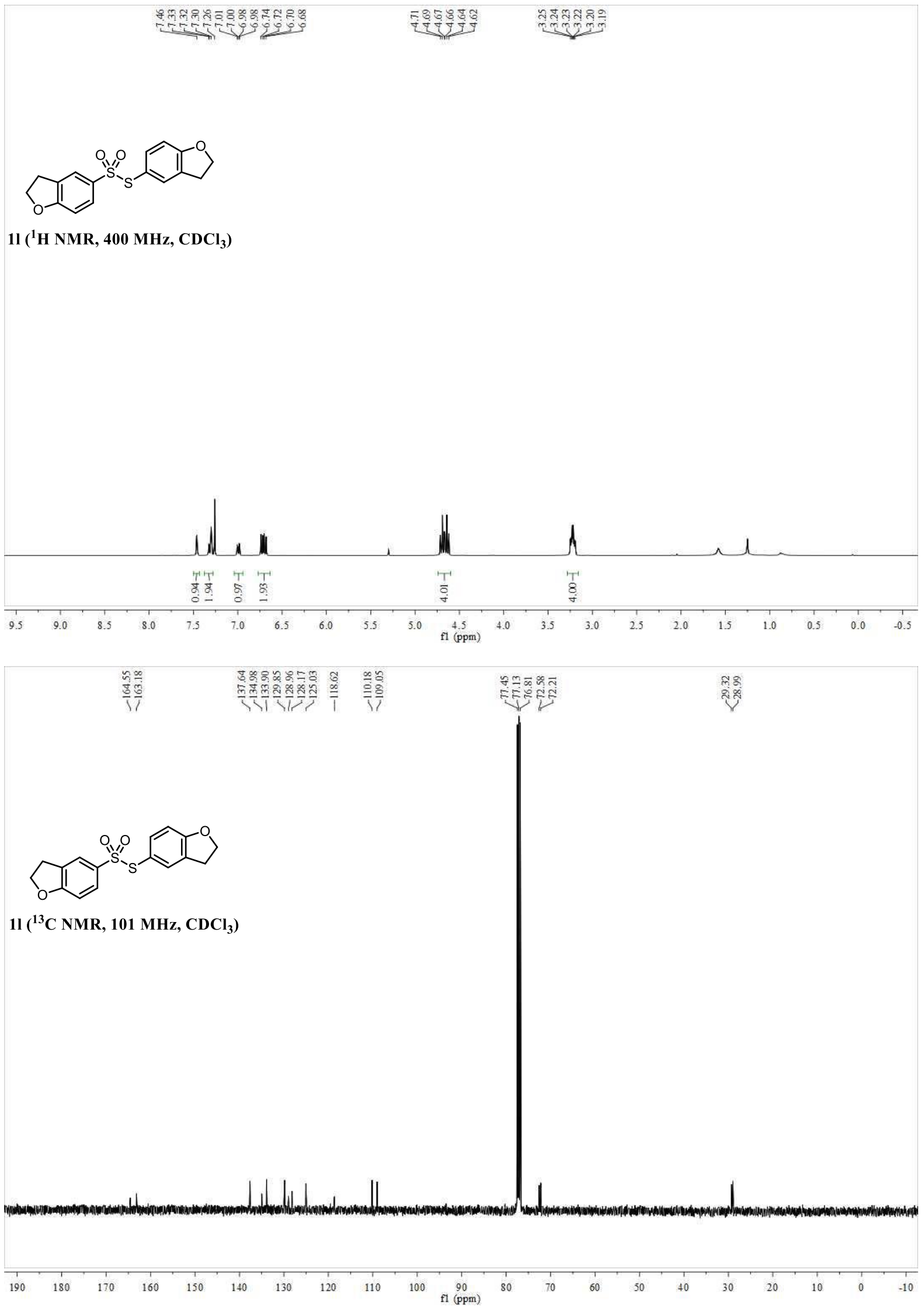


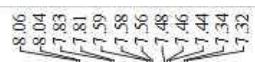

聯乎

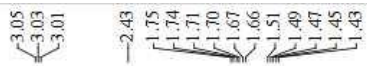
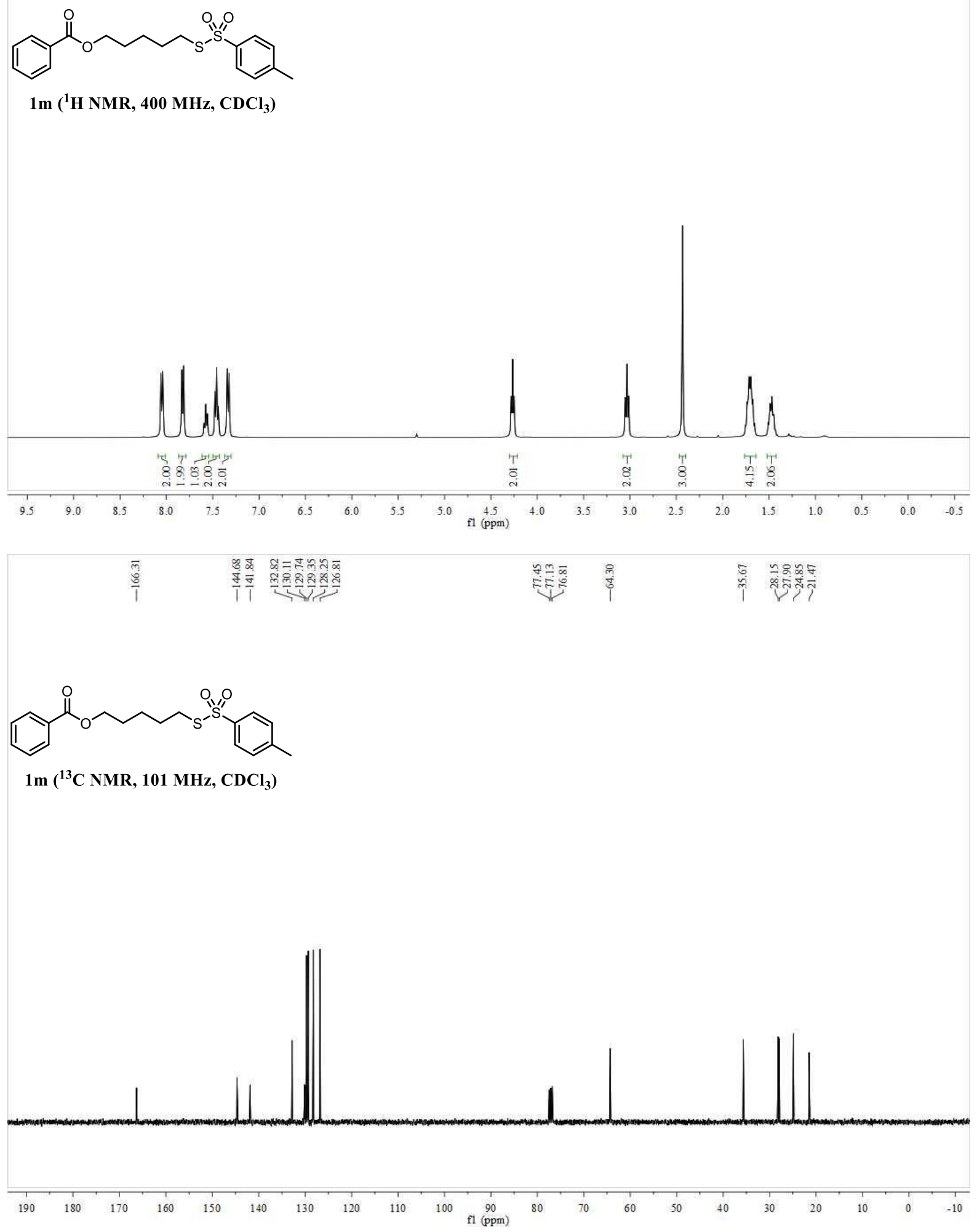

S22 

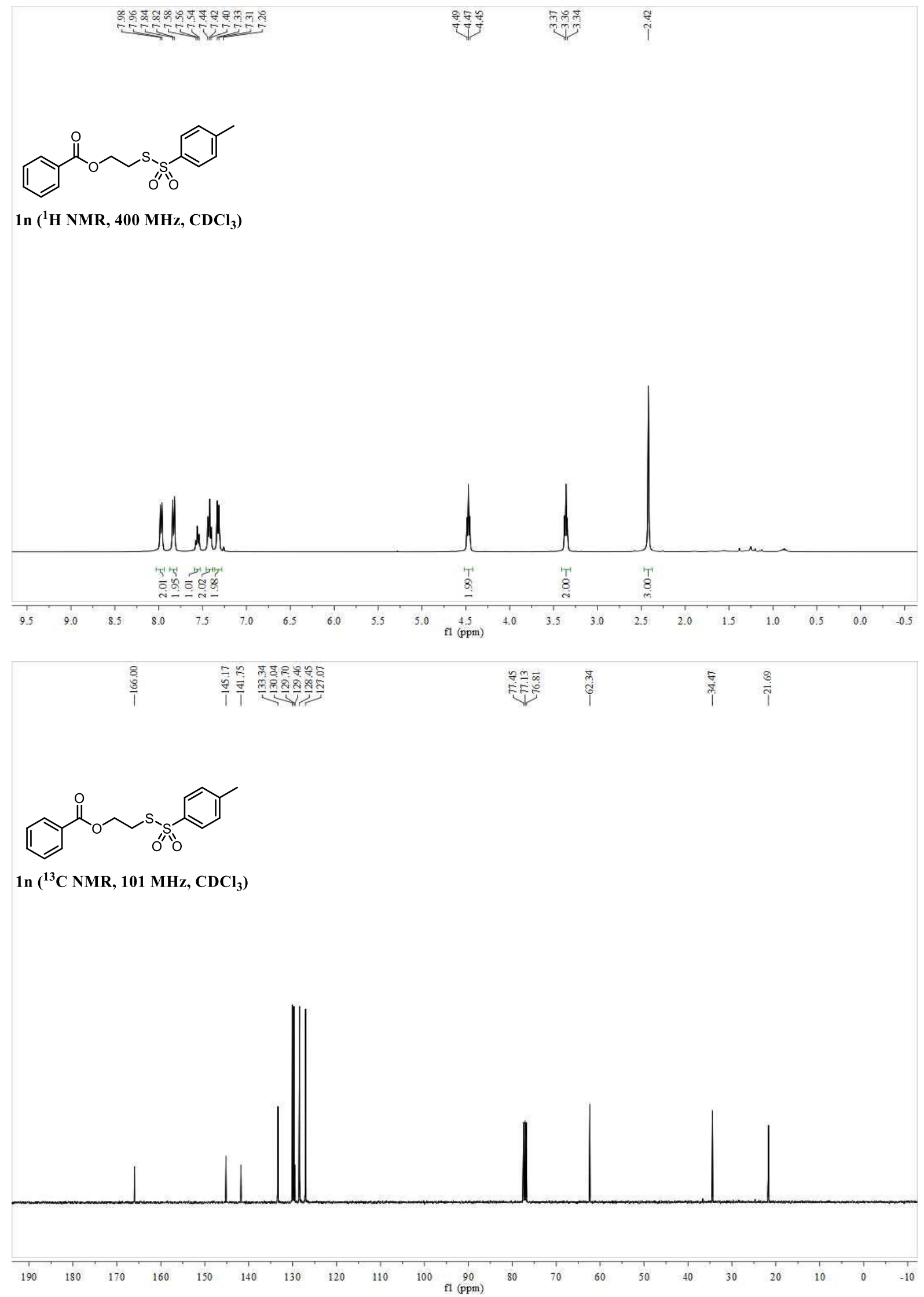

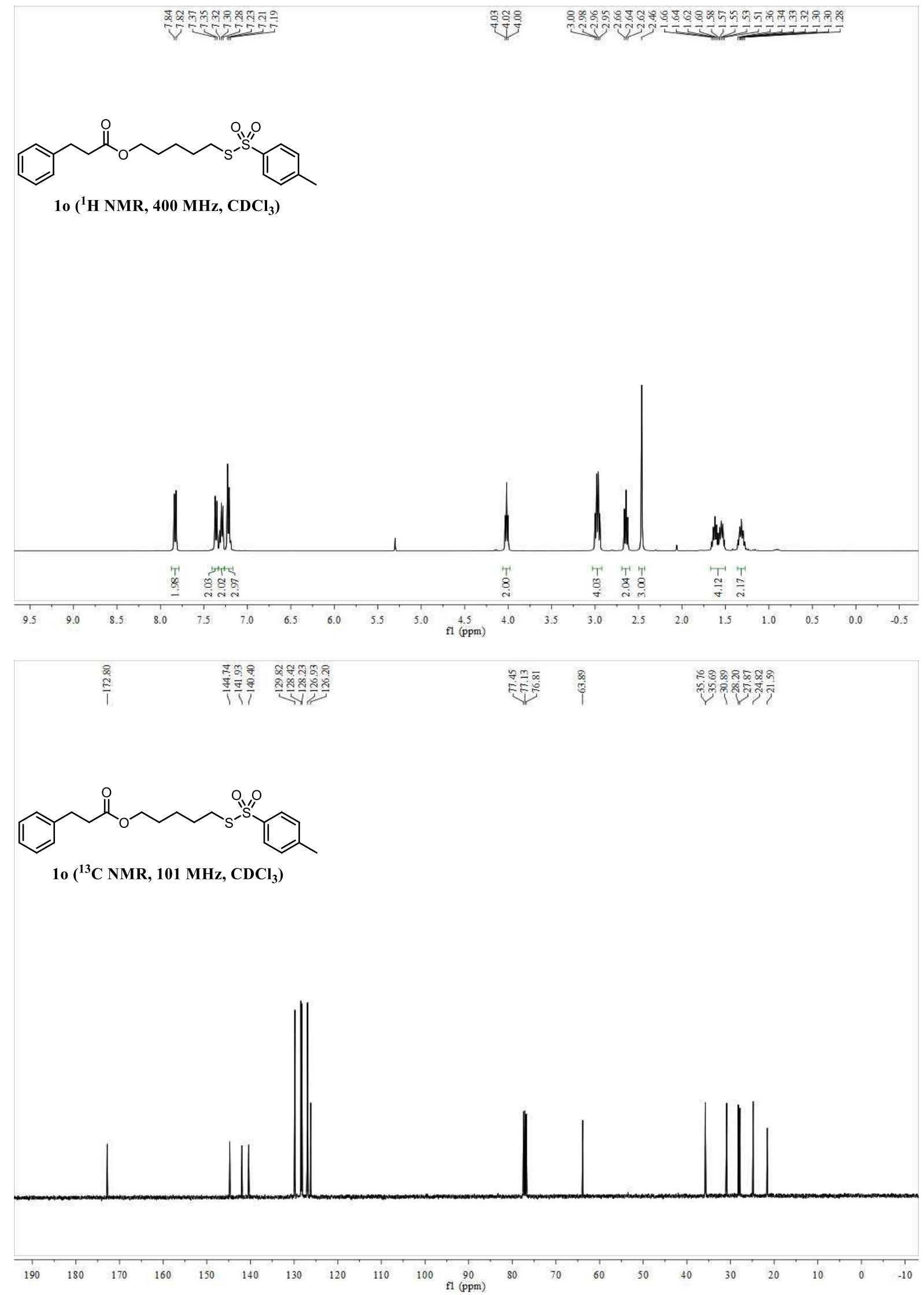

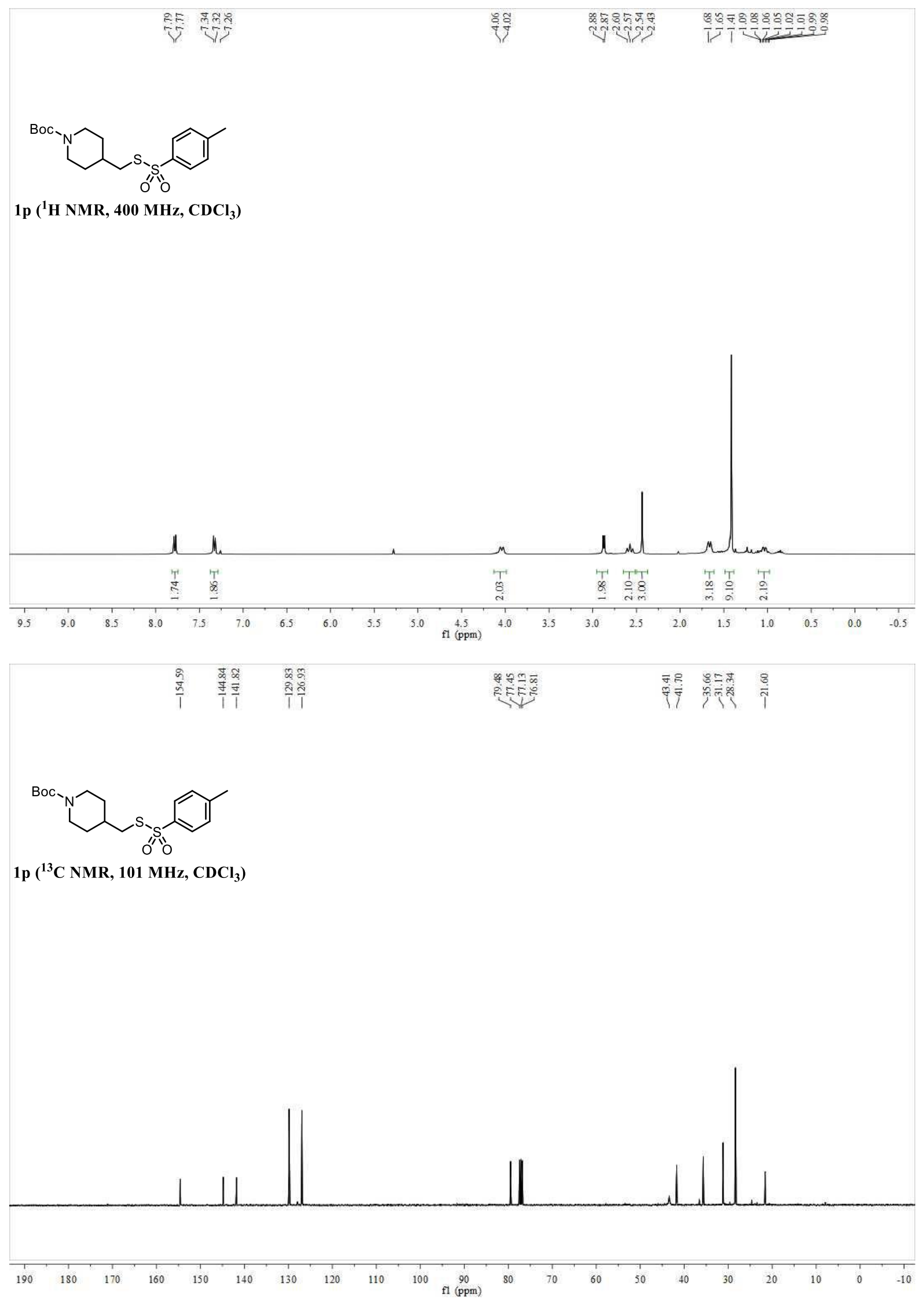

S25 

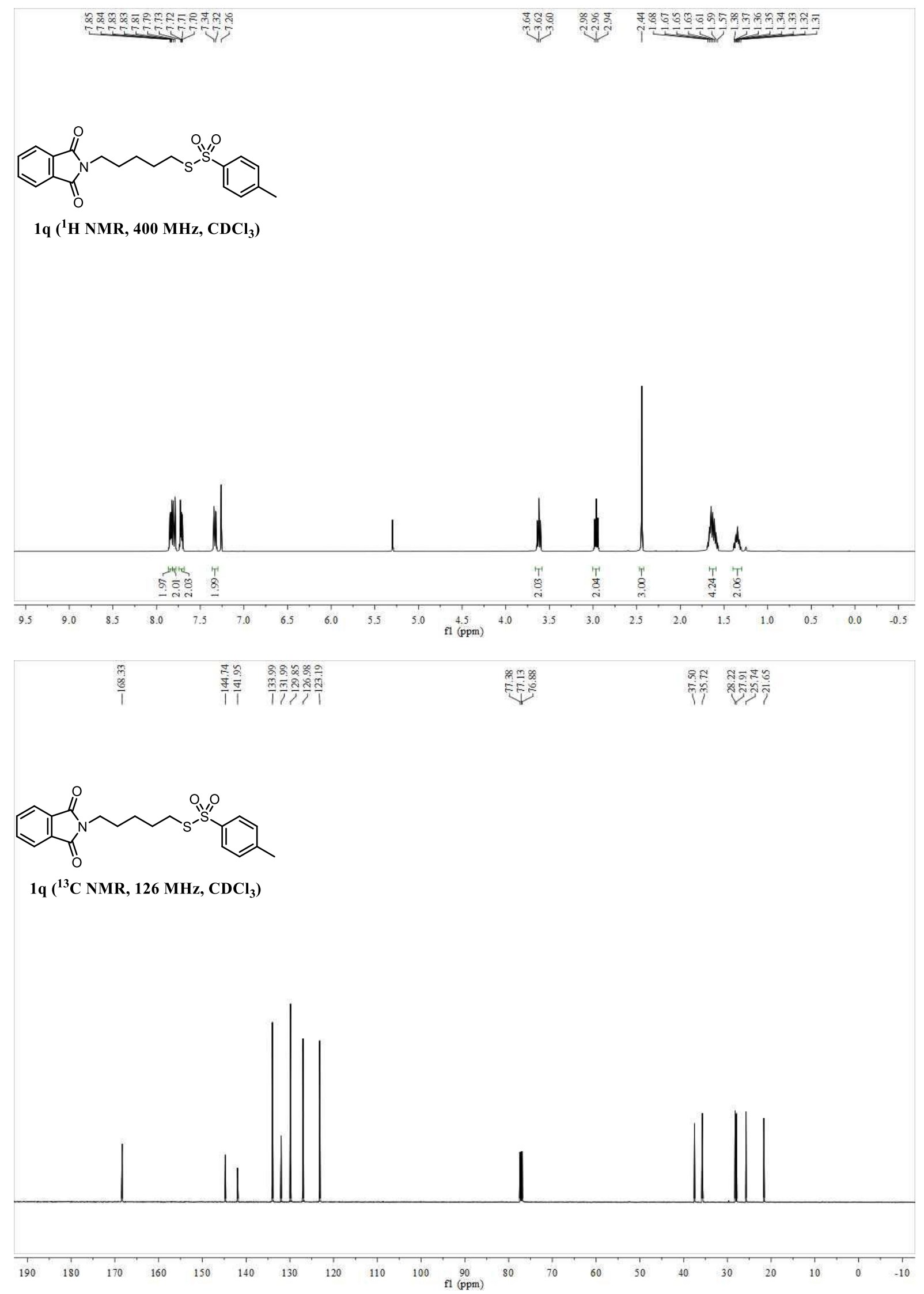

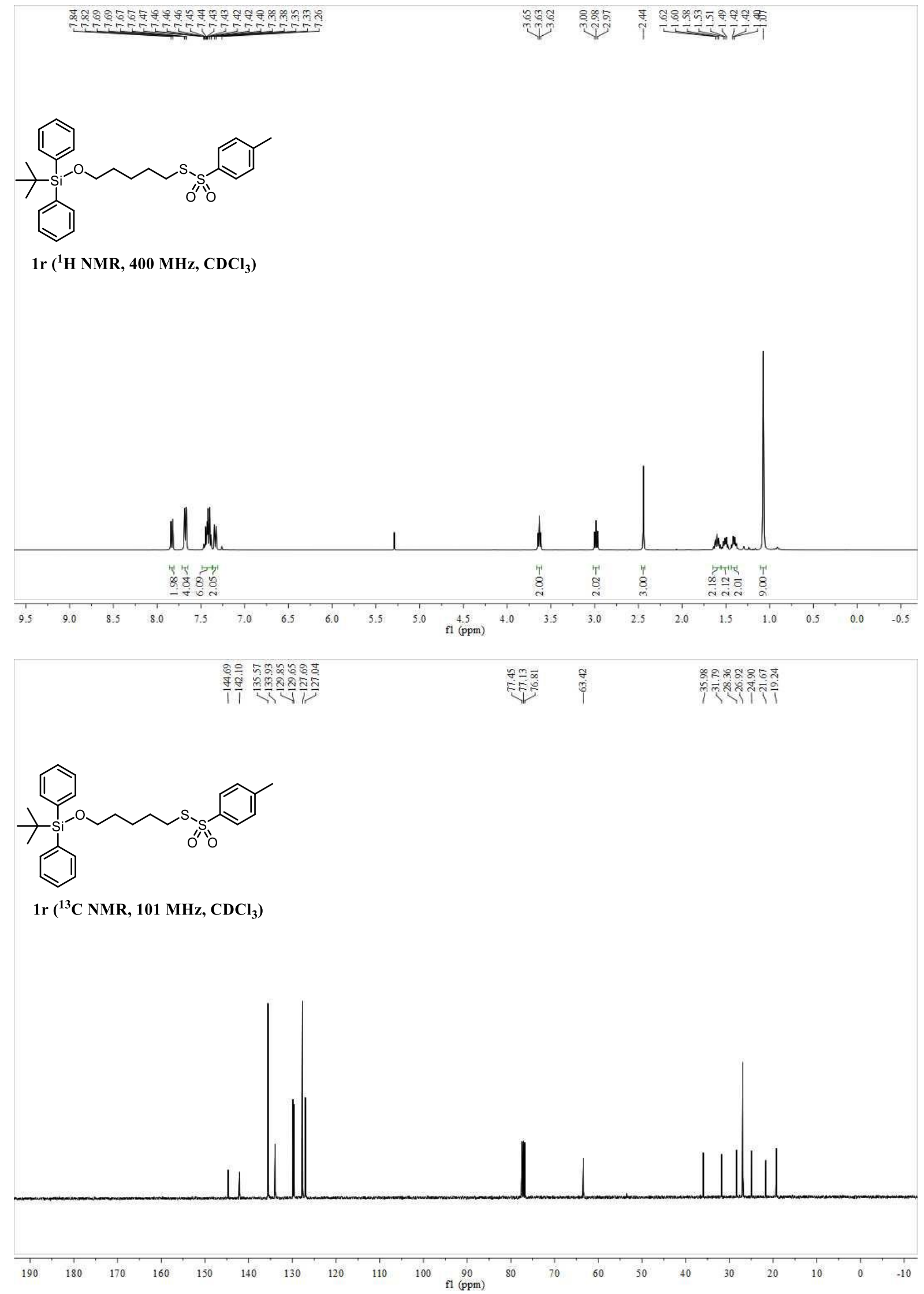


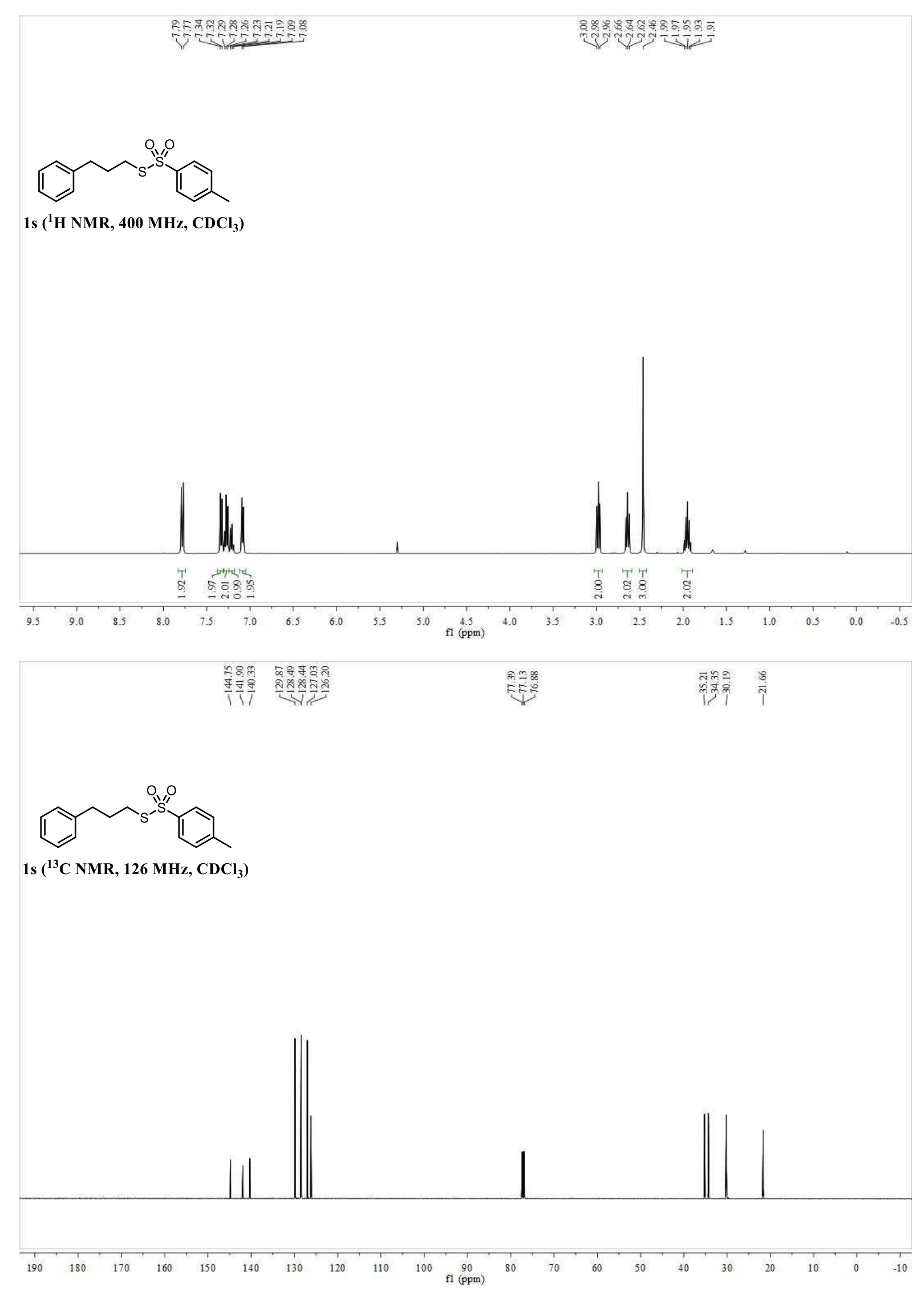




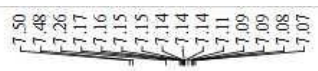

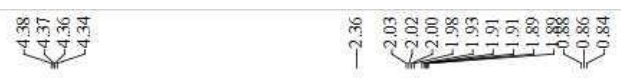

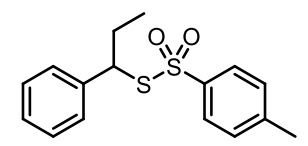

1u ( ${ }^{1} \mathrm{H}$ NMR, $\left.400 \mathrm{MHz}, \mathrm{CDCl}_{3}\right)$
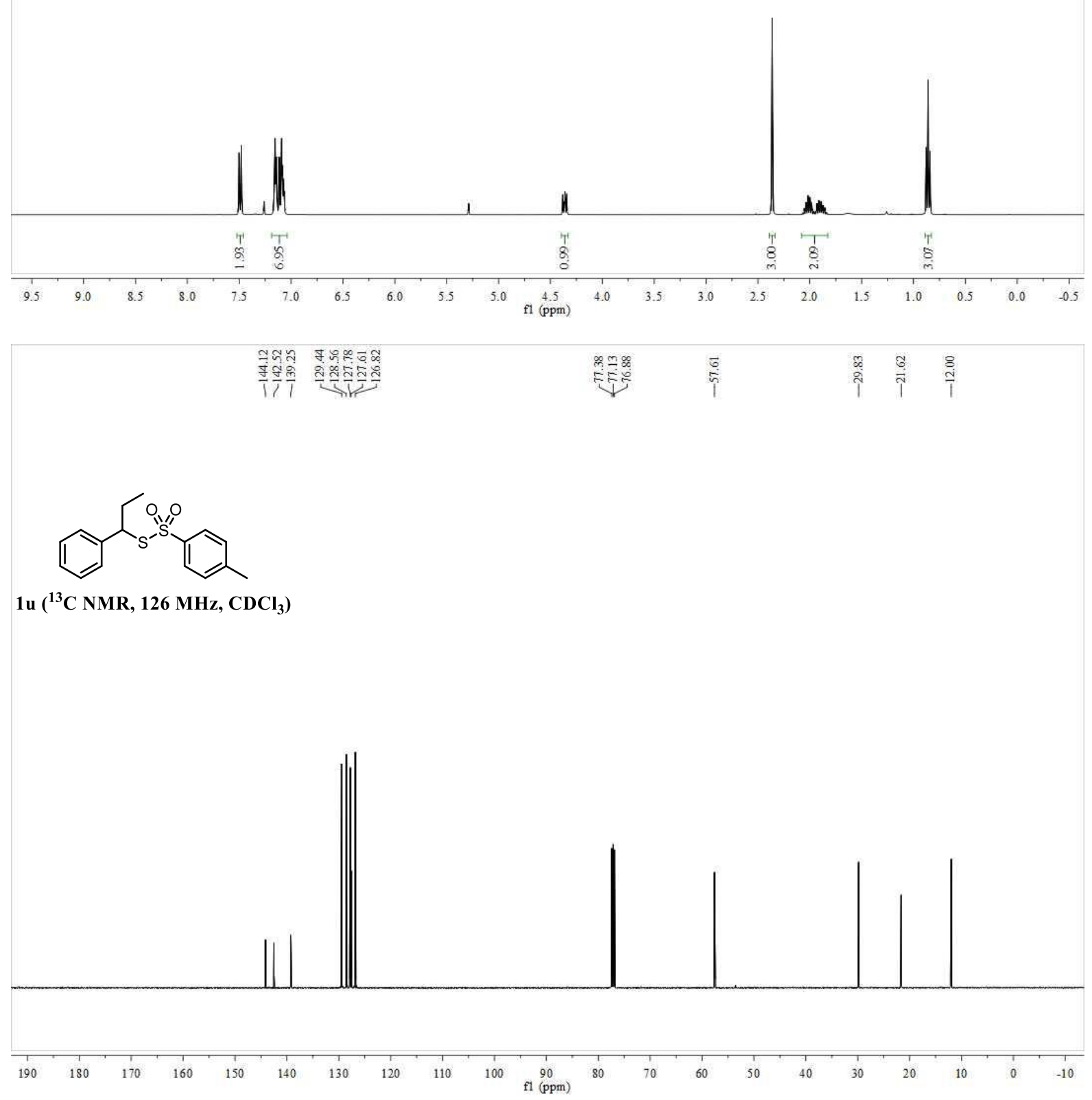

S29 

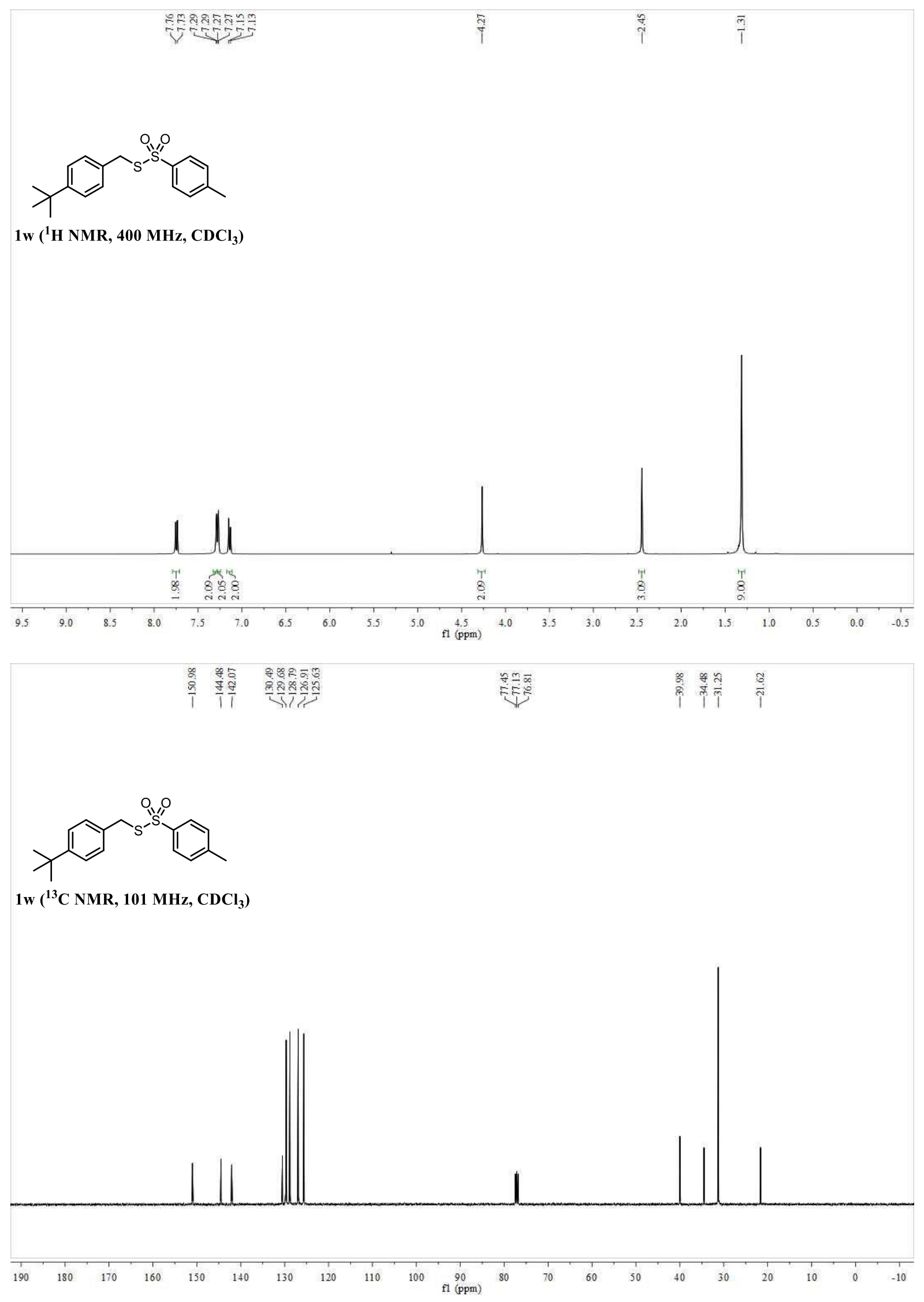

S30 


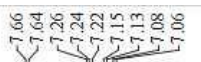

$\frac{9}{7}$

$\underset{i}{\vec{i}}$
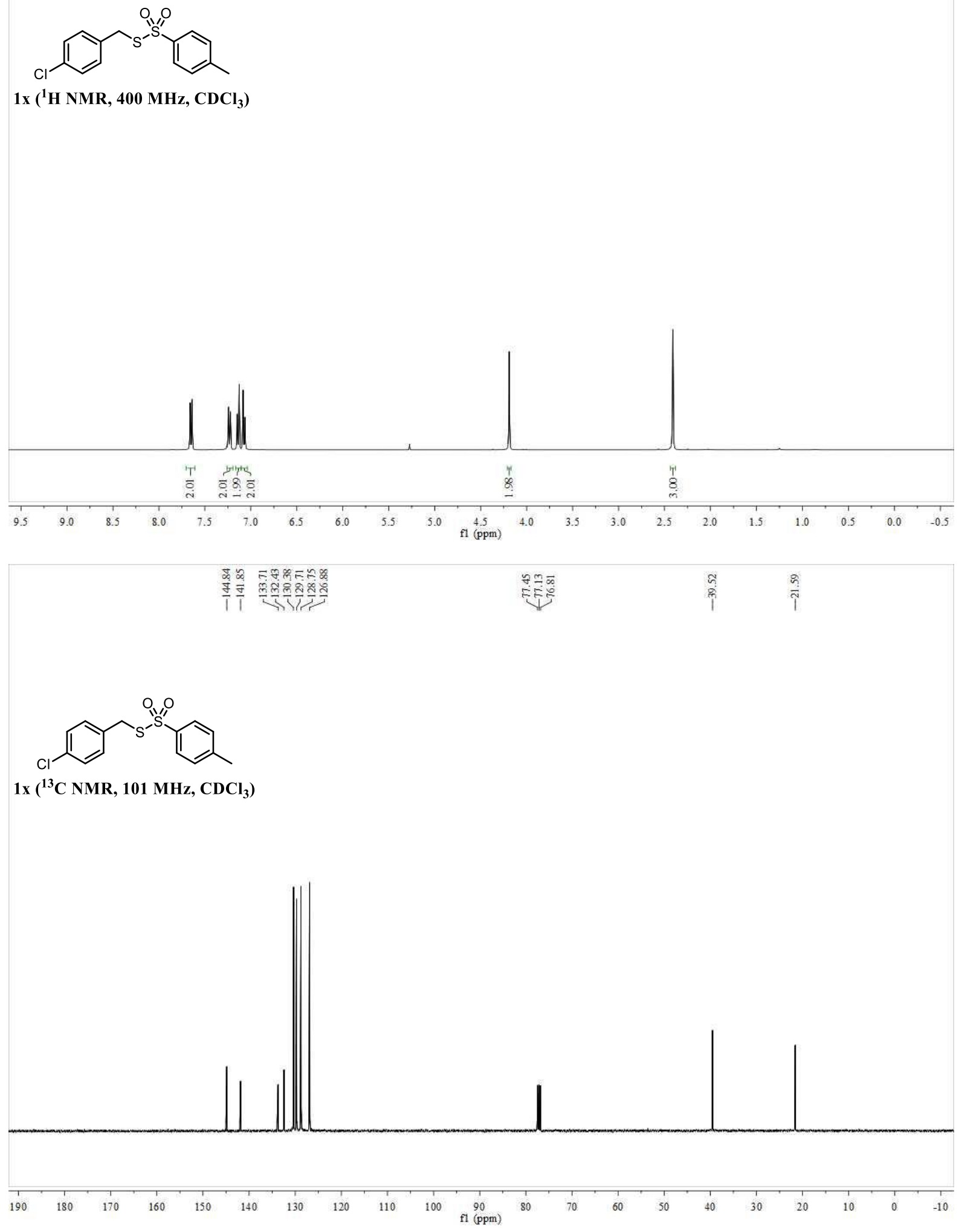

S31 

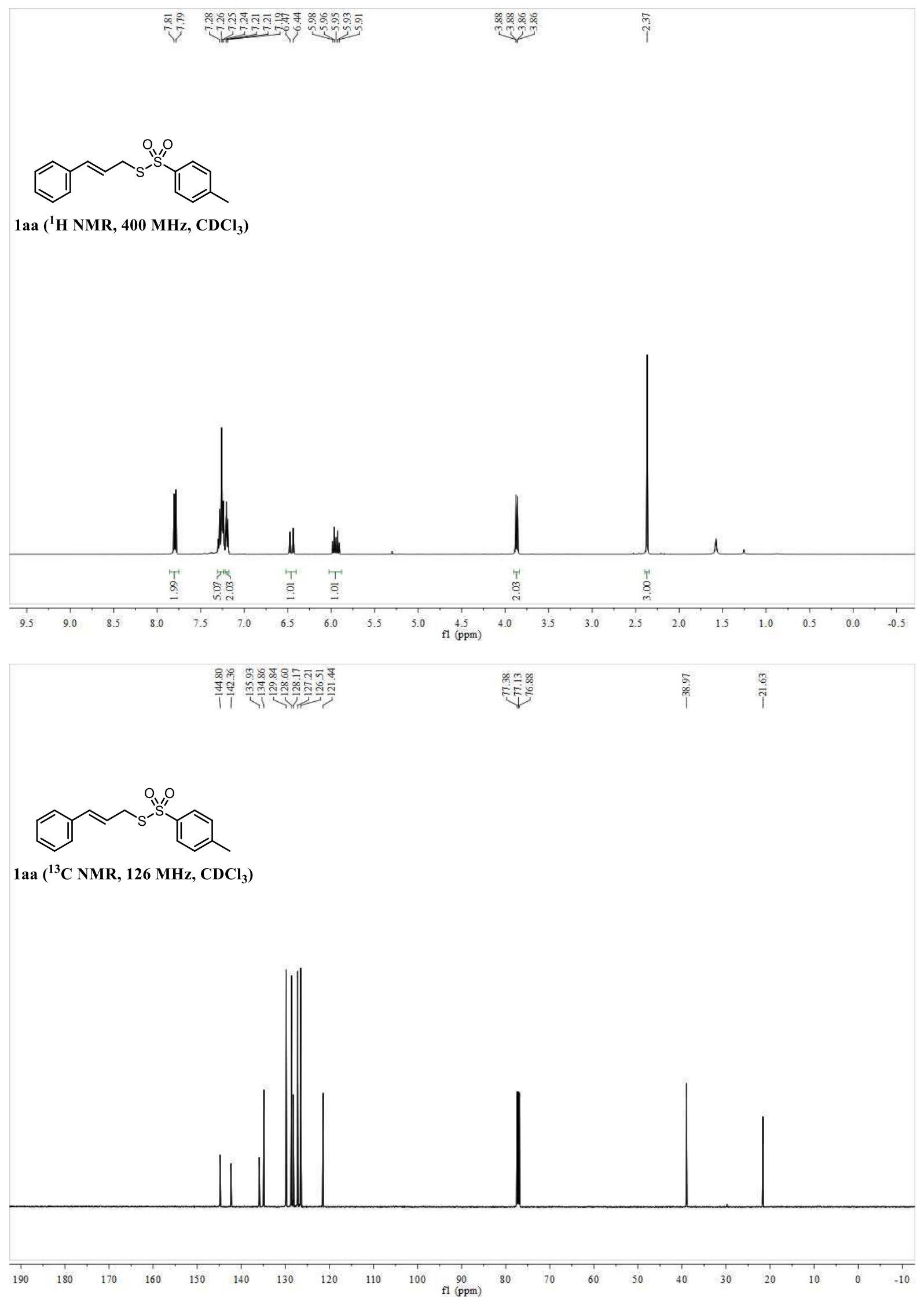

S32 
2.

$\stackrel{8}{1} \quad \stackrel{8}{1}$

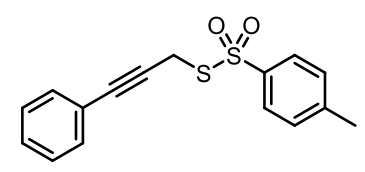

$1 \mathrm{ab}\left({ }^{1} \mathrm{H}\right.$ NMR, $400 \mathrm{MHz}, \mathrm{CDCl}_{3}$ )
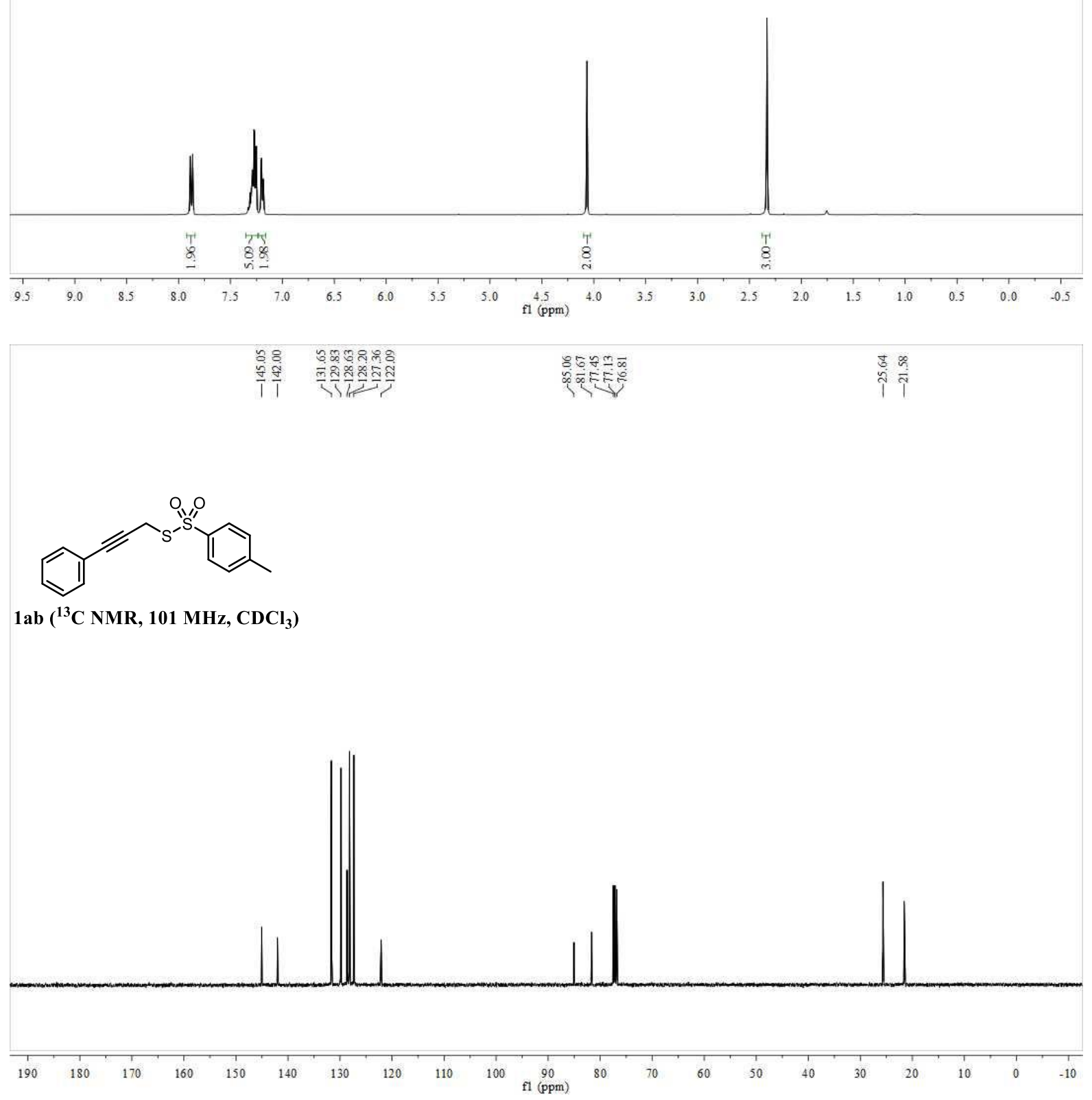

S33 

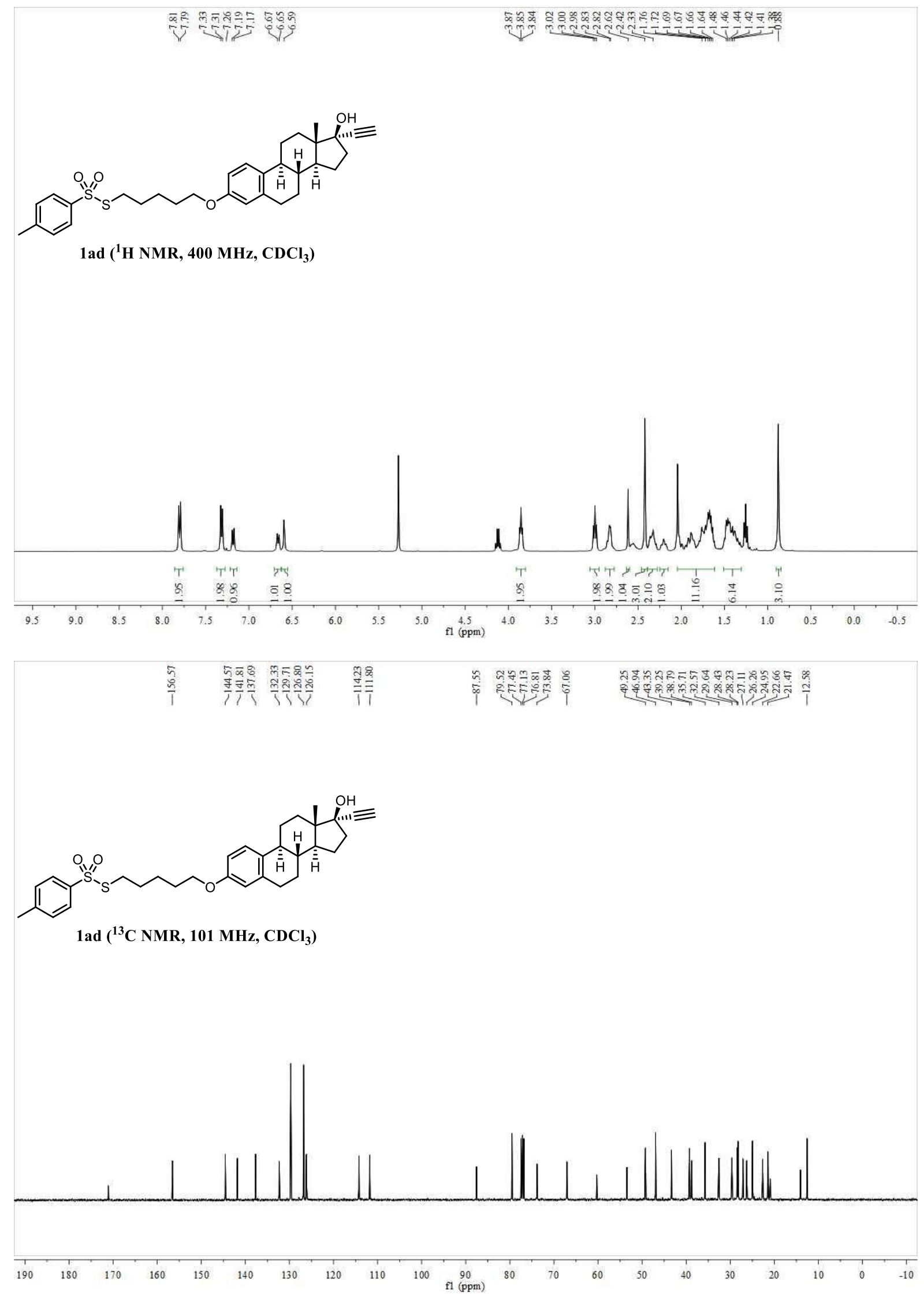

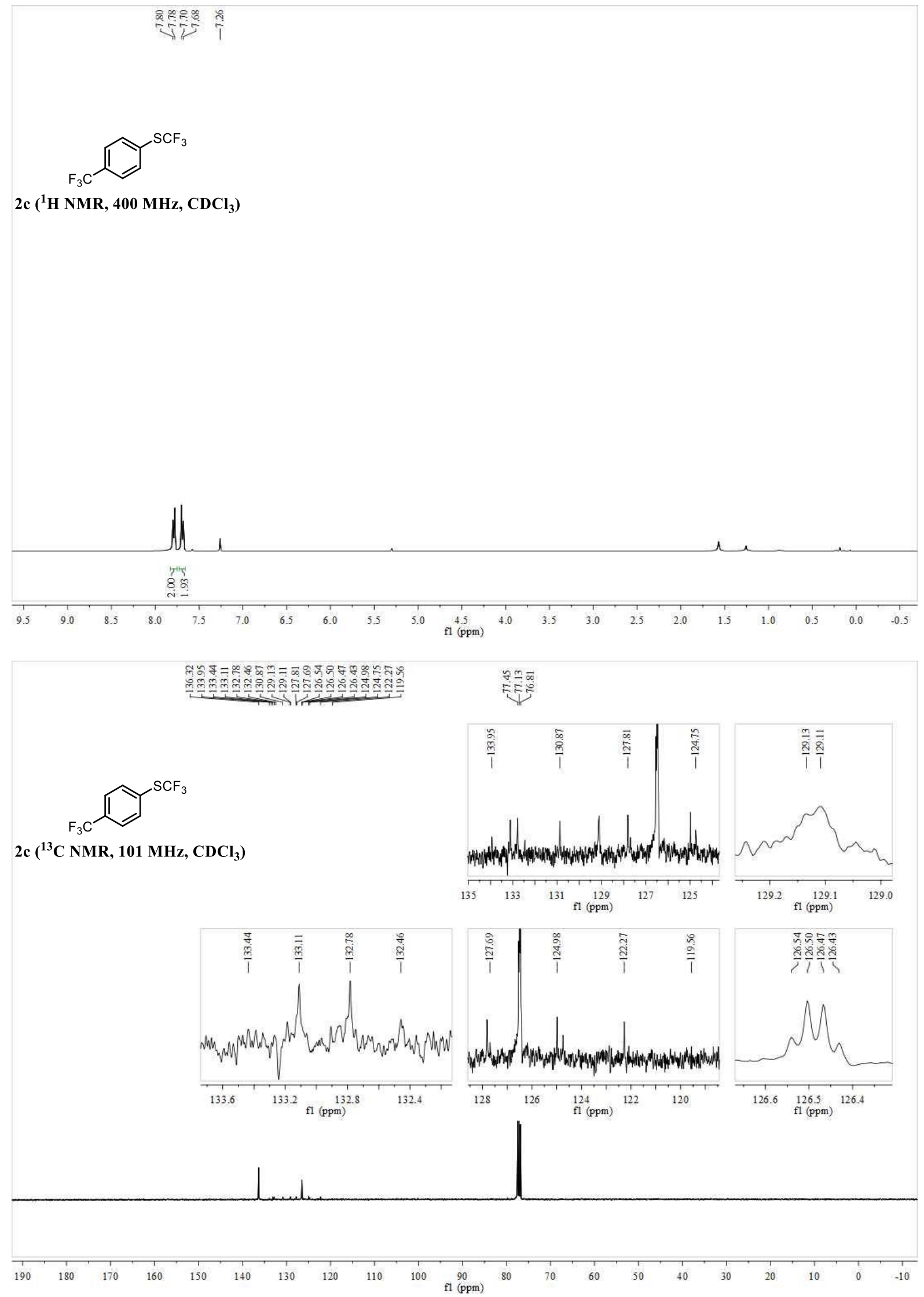

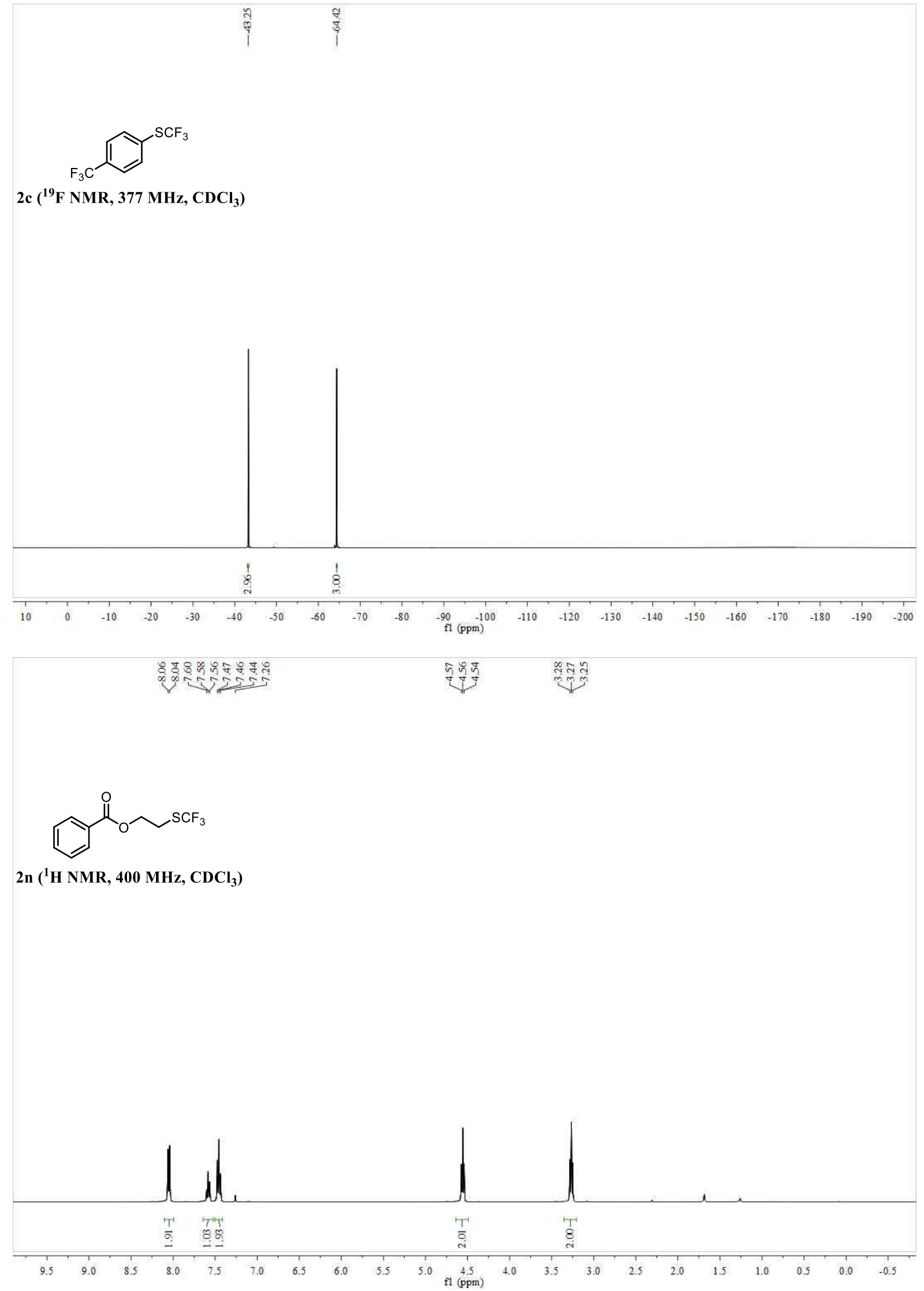

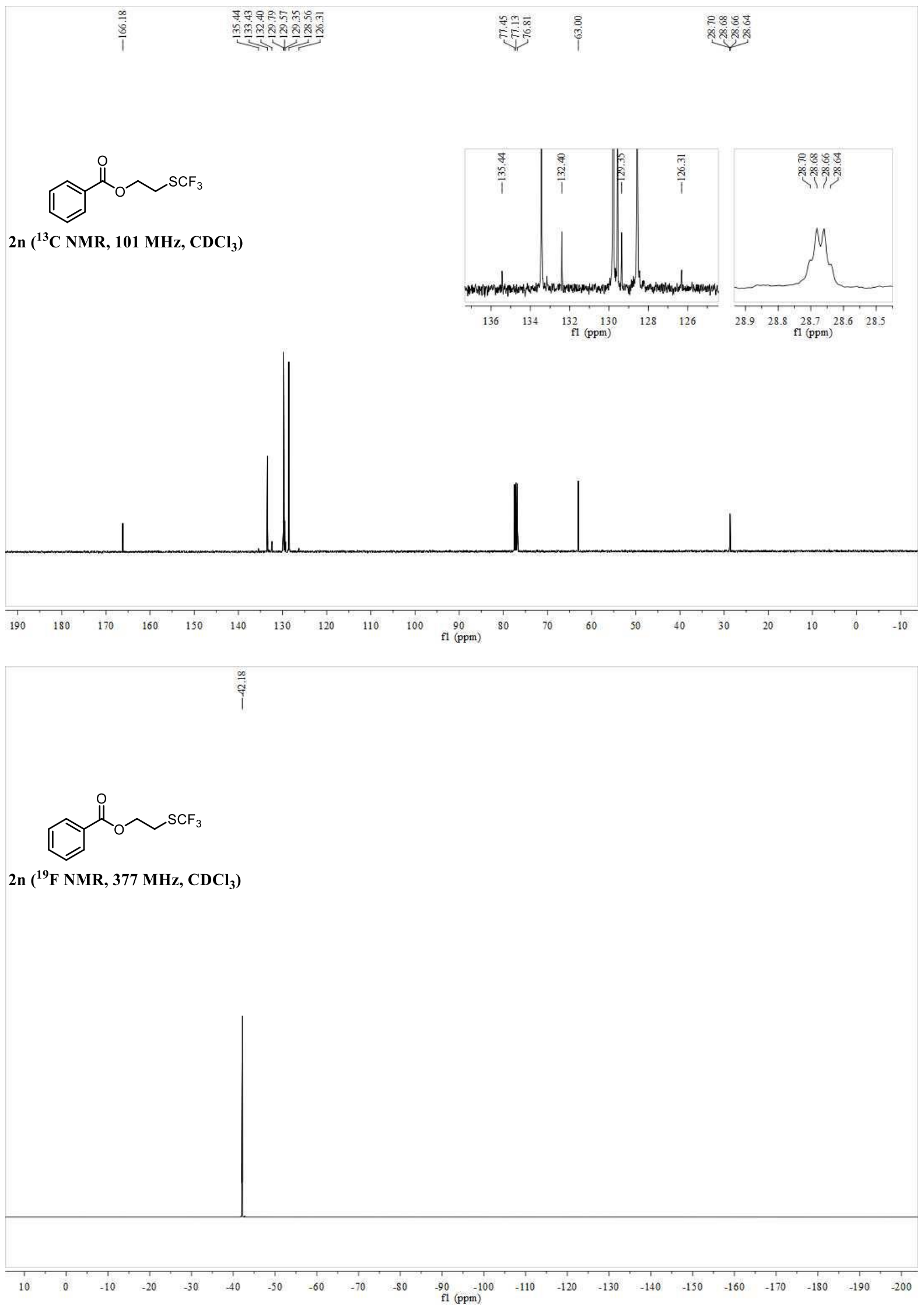

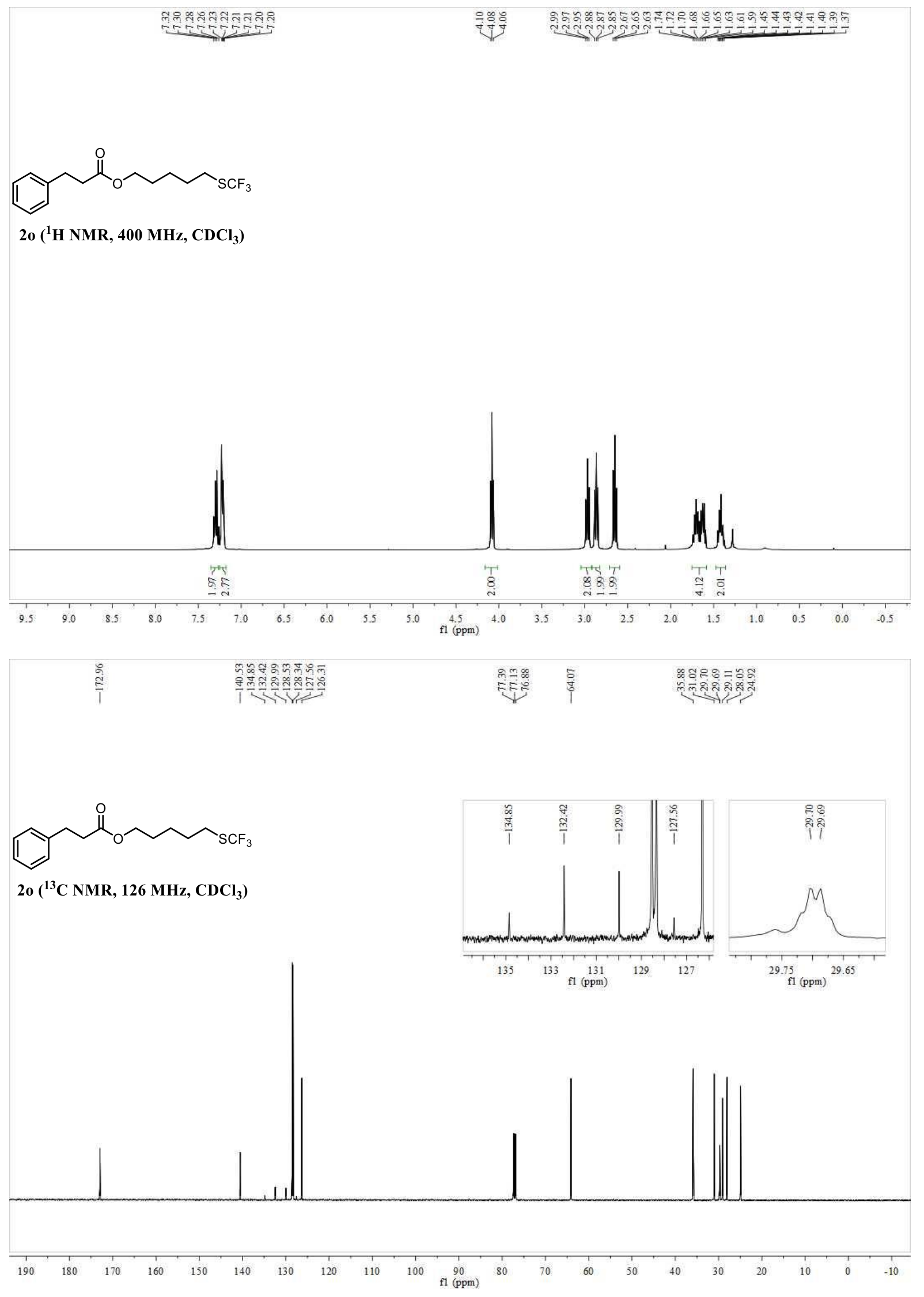

S38 

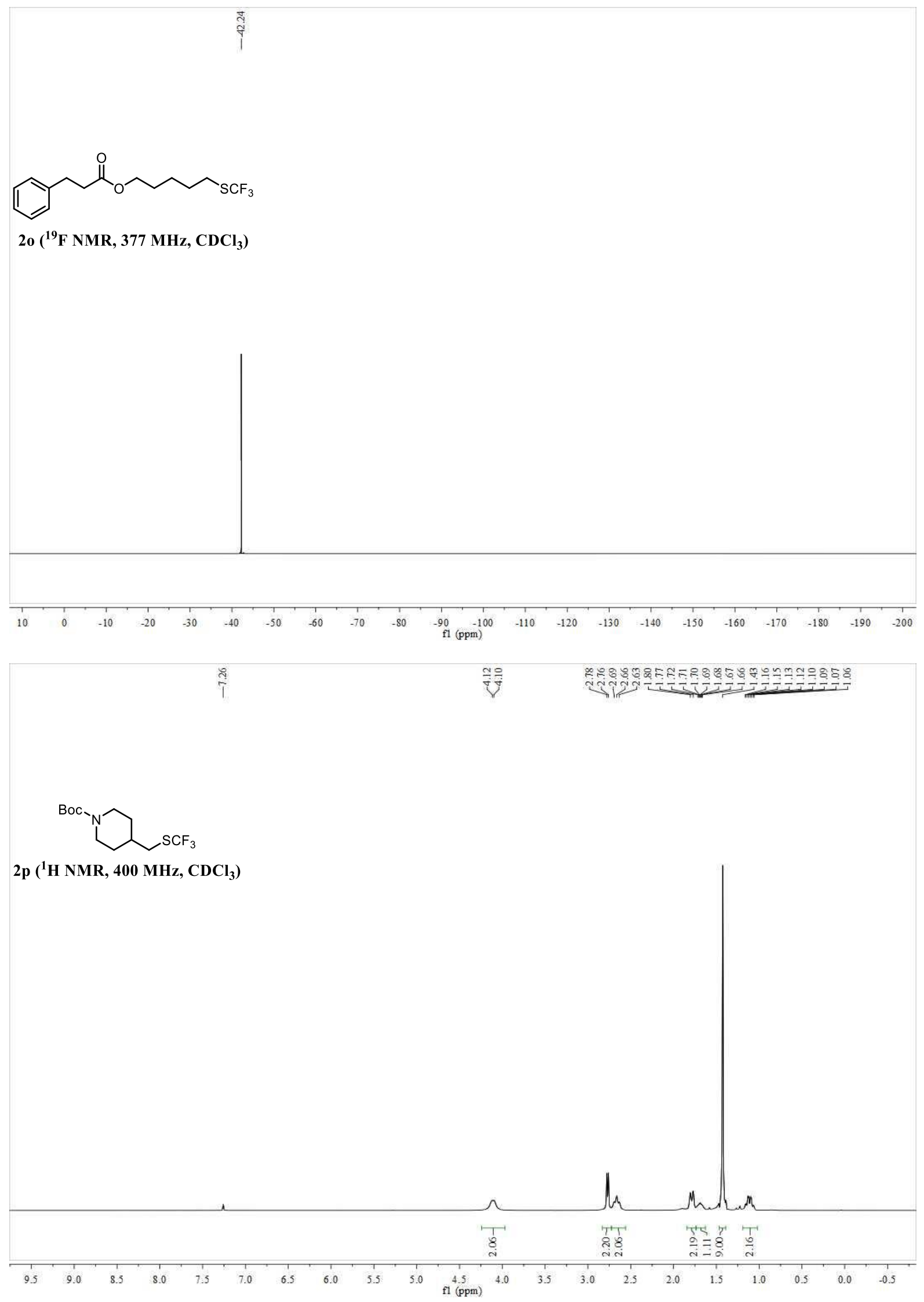

S39 


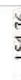

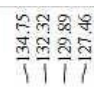

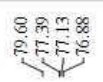

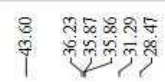

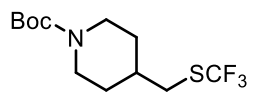

2p $\left({ }^{13} \mathrm{C} \mathrm{NMR,} 126 \mathrm{MHz}^{\mathrm{CDCl}}{ }_{3}\right)$
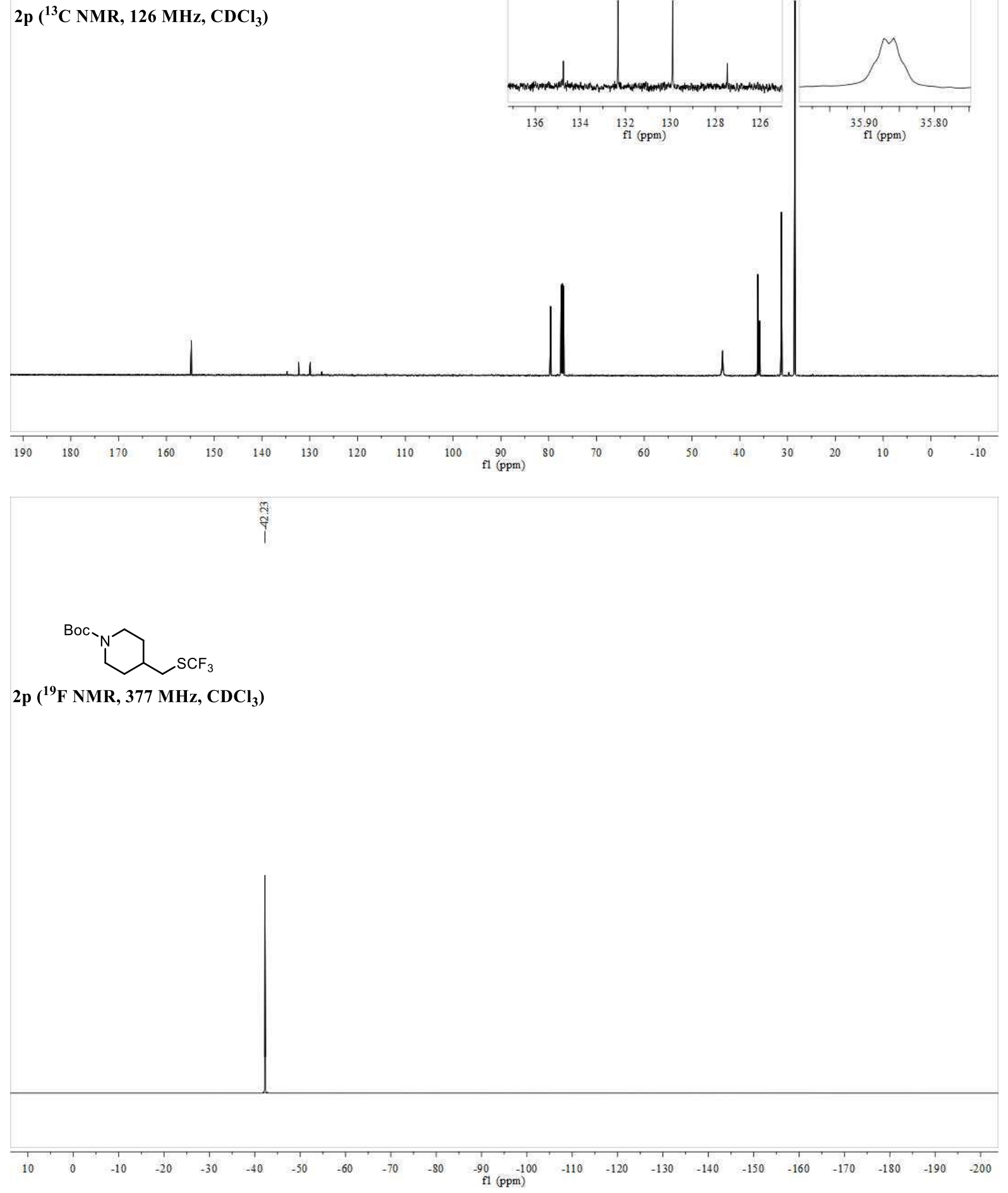

S40 


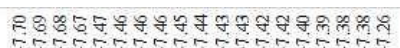

ํㅐㅁ

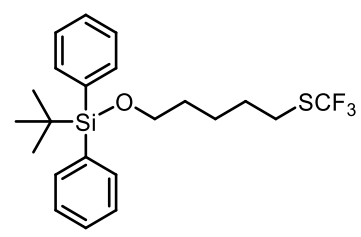

2r $\left({ }^{1} \mathrm{H}\right.$ NMR, $\left.400 \mathrm{MHz}, \mathrm{CDCl}_{3}\right)$

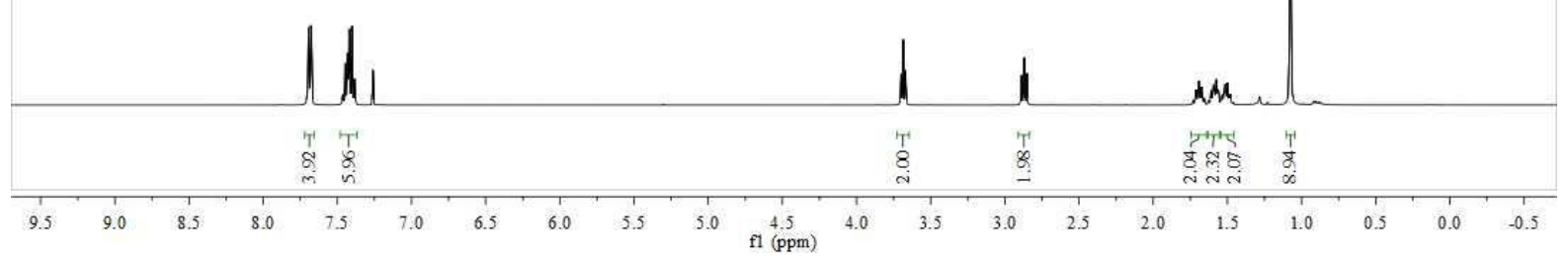

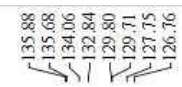

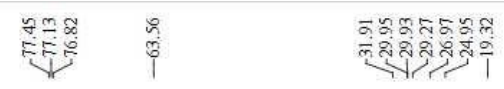

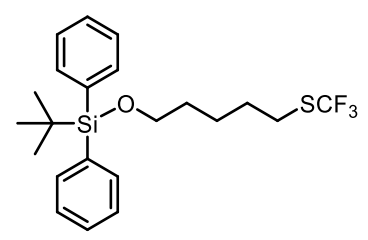

2r $\left({ }^{13} \mathrm{C}\right.$ NMR, $\left.101 \mathrm{MHz}, \mathrm{CDCl}_{3}\right)$
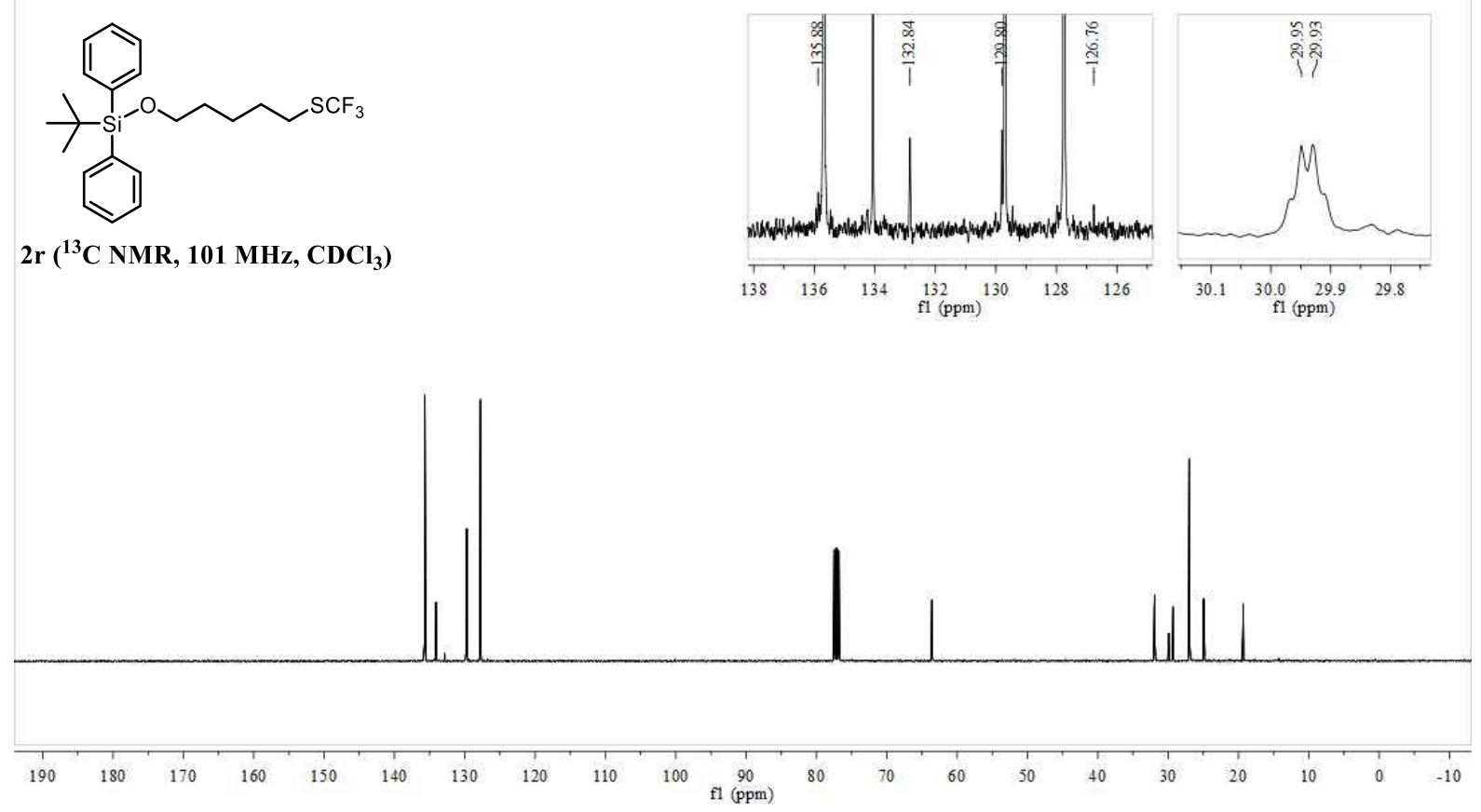

S41 

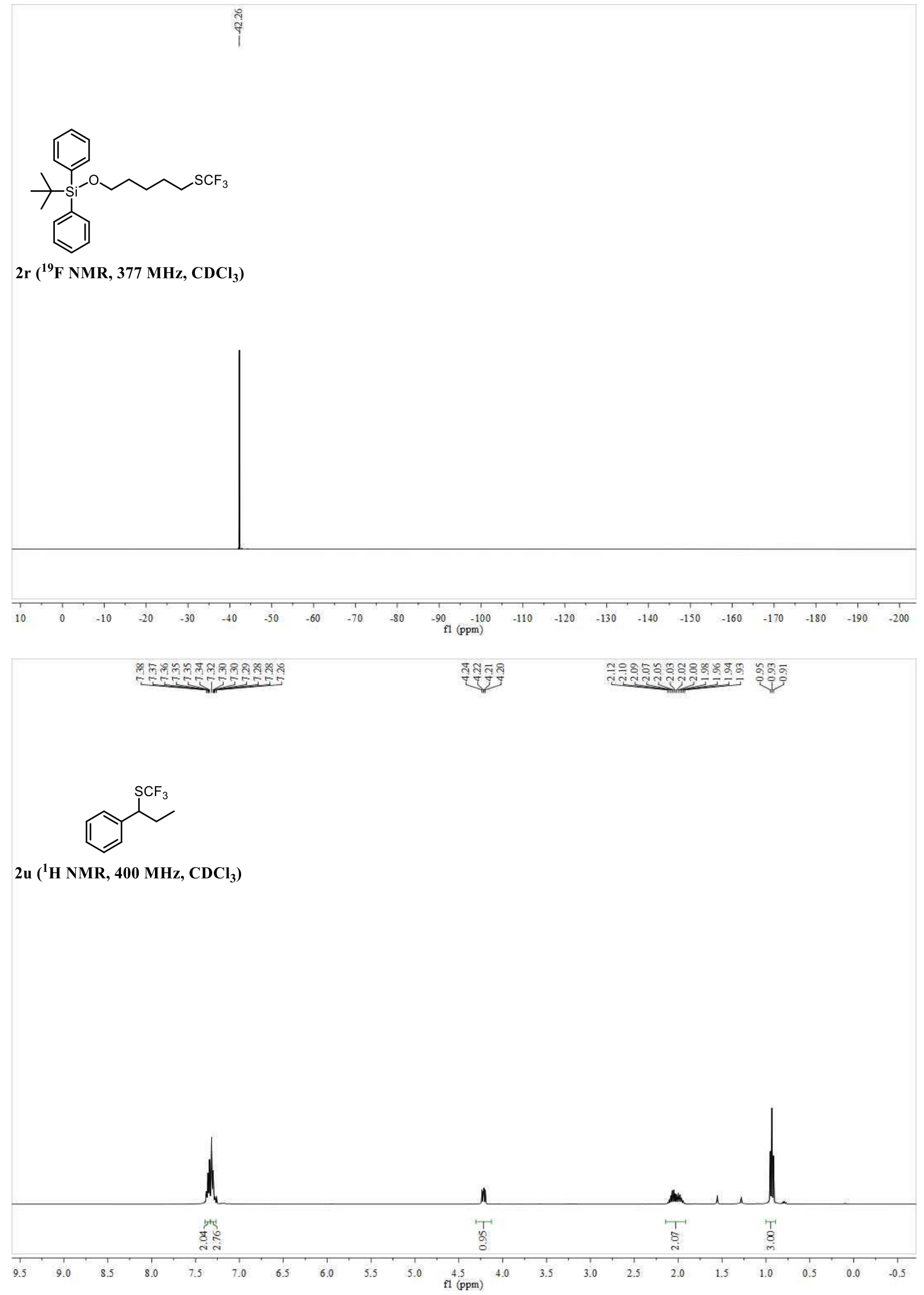

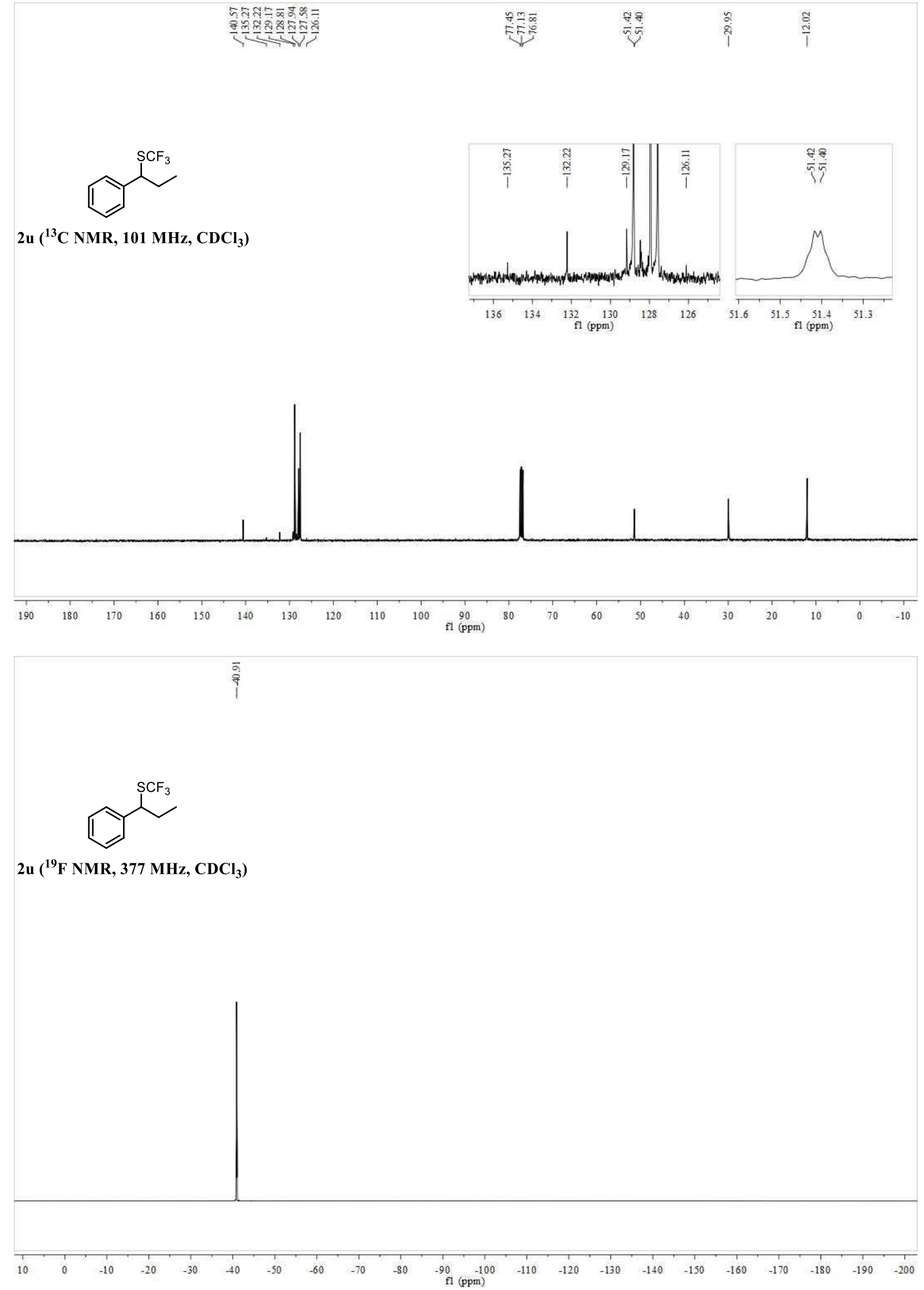

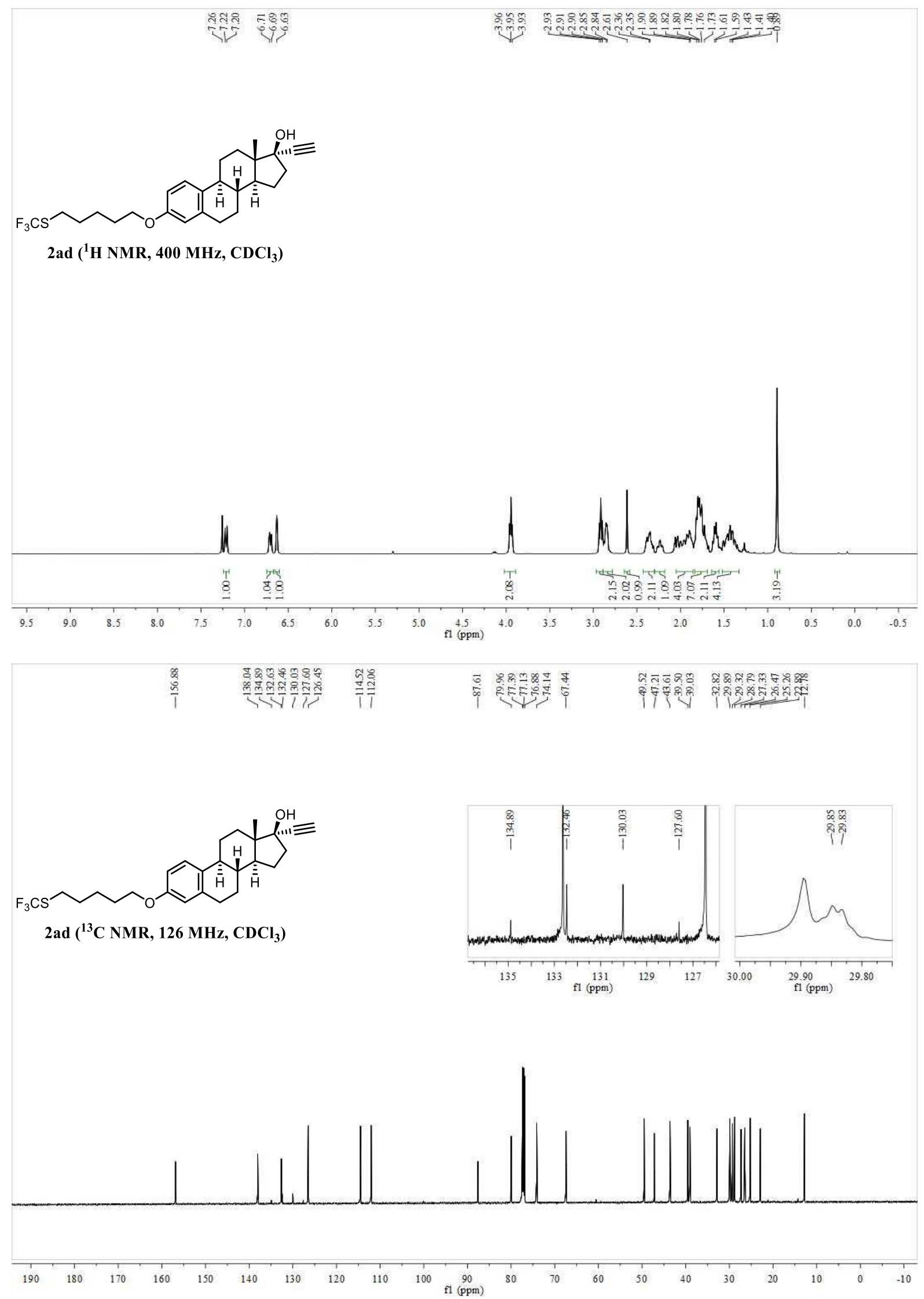

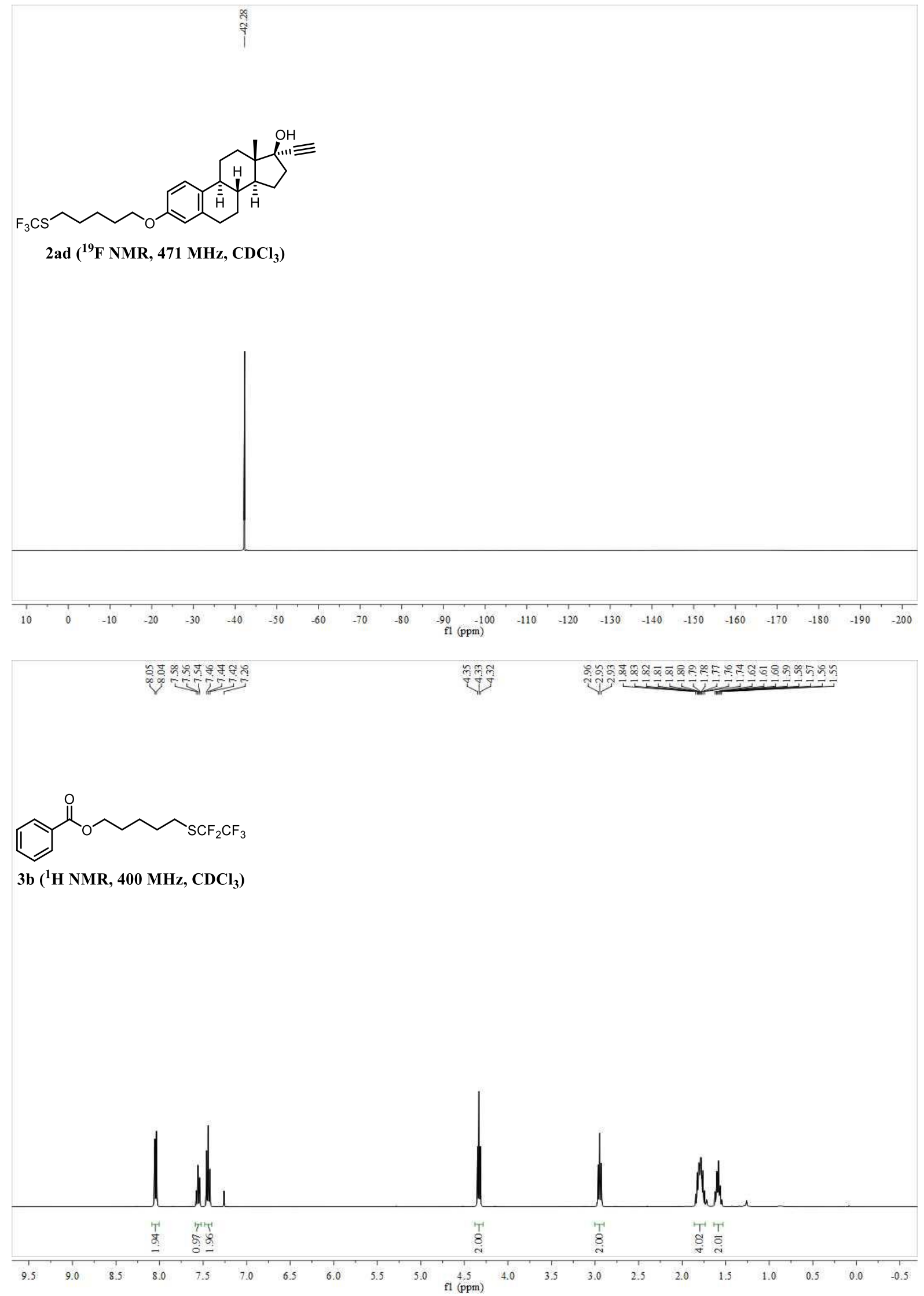


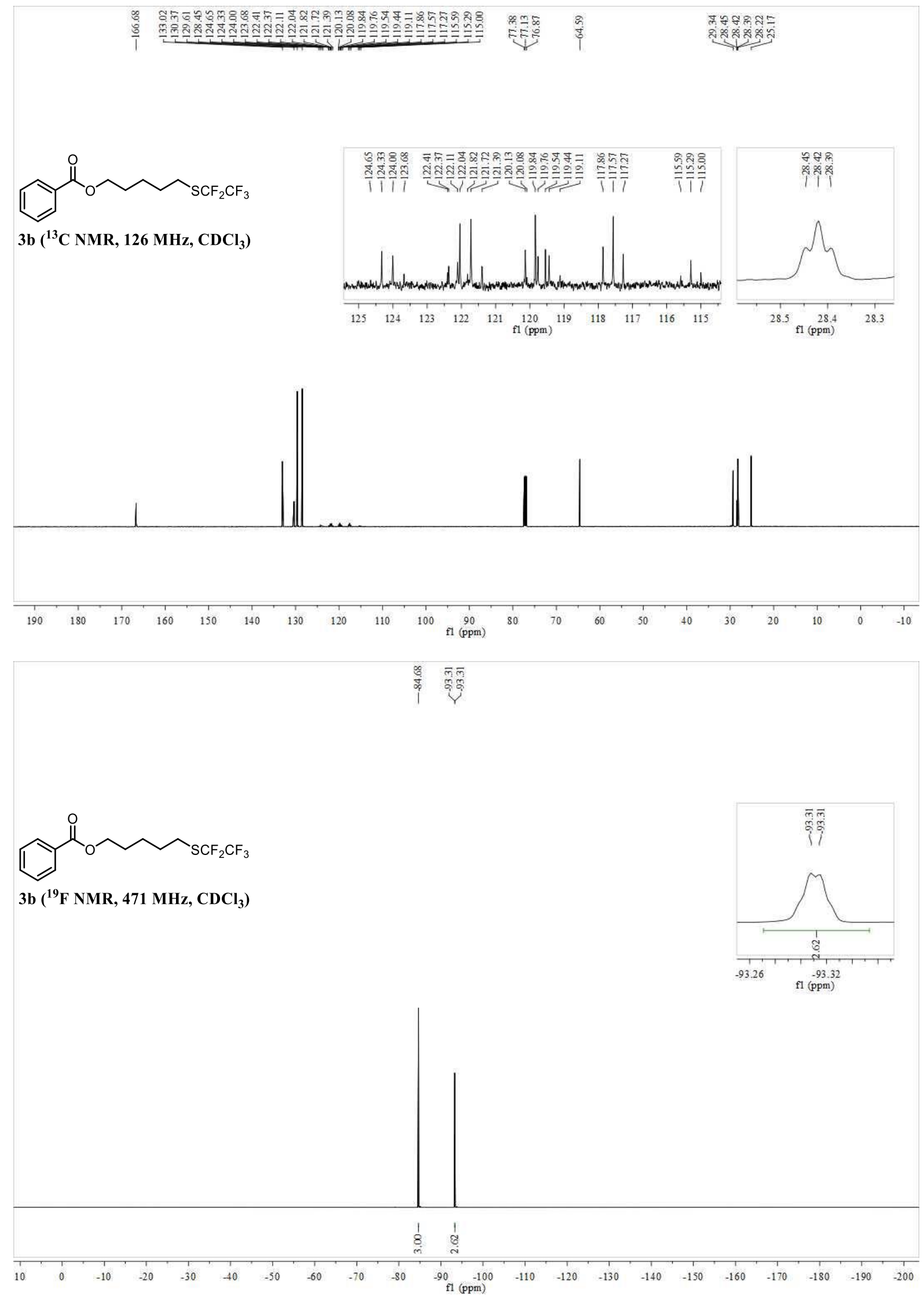



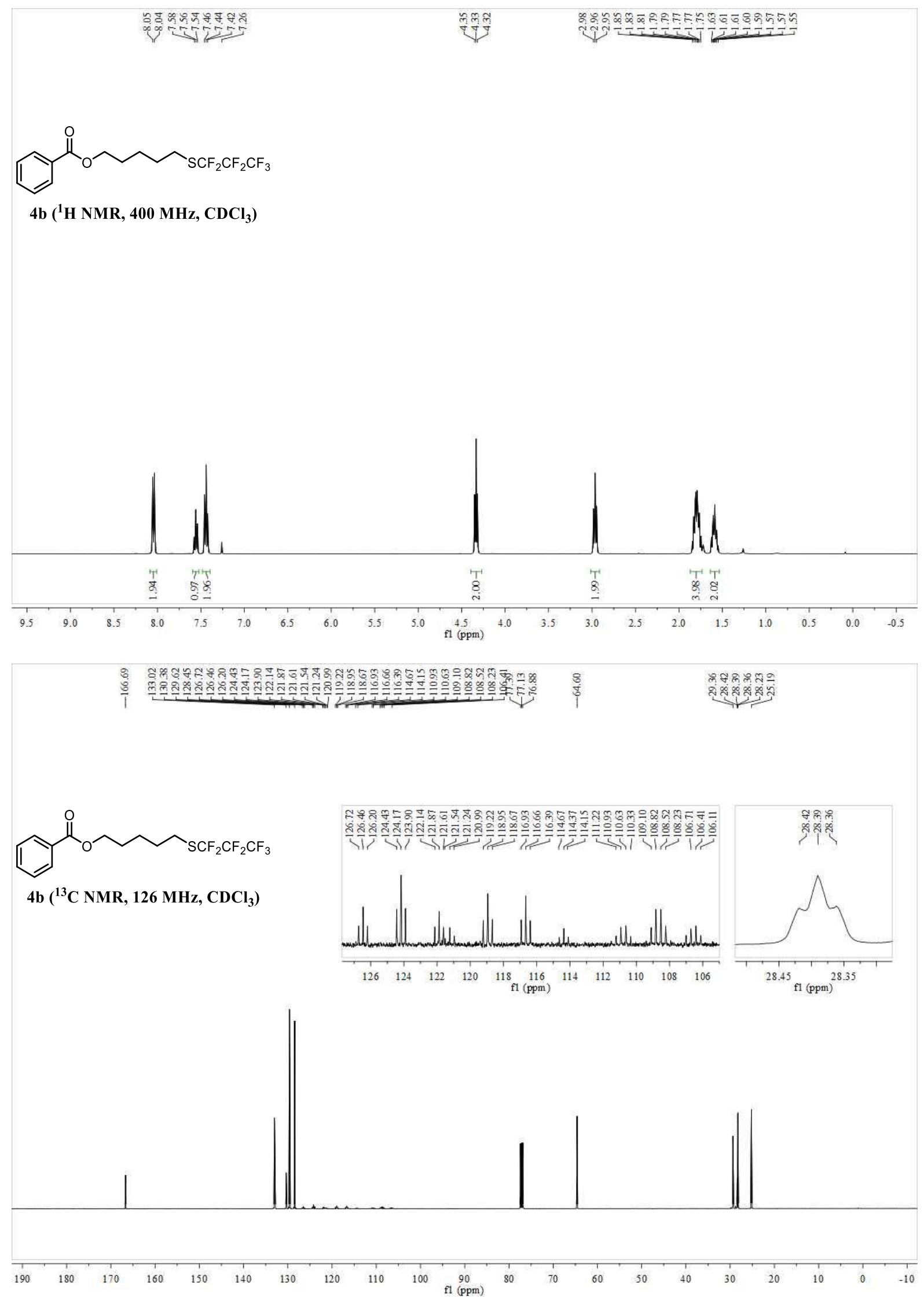


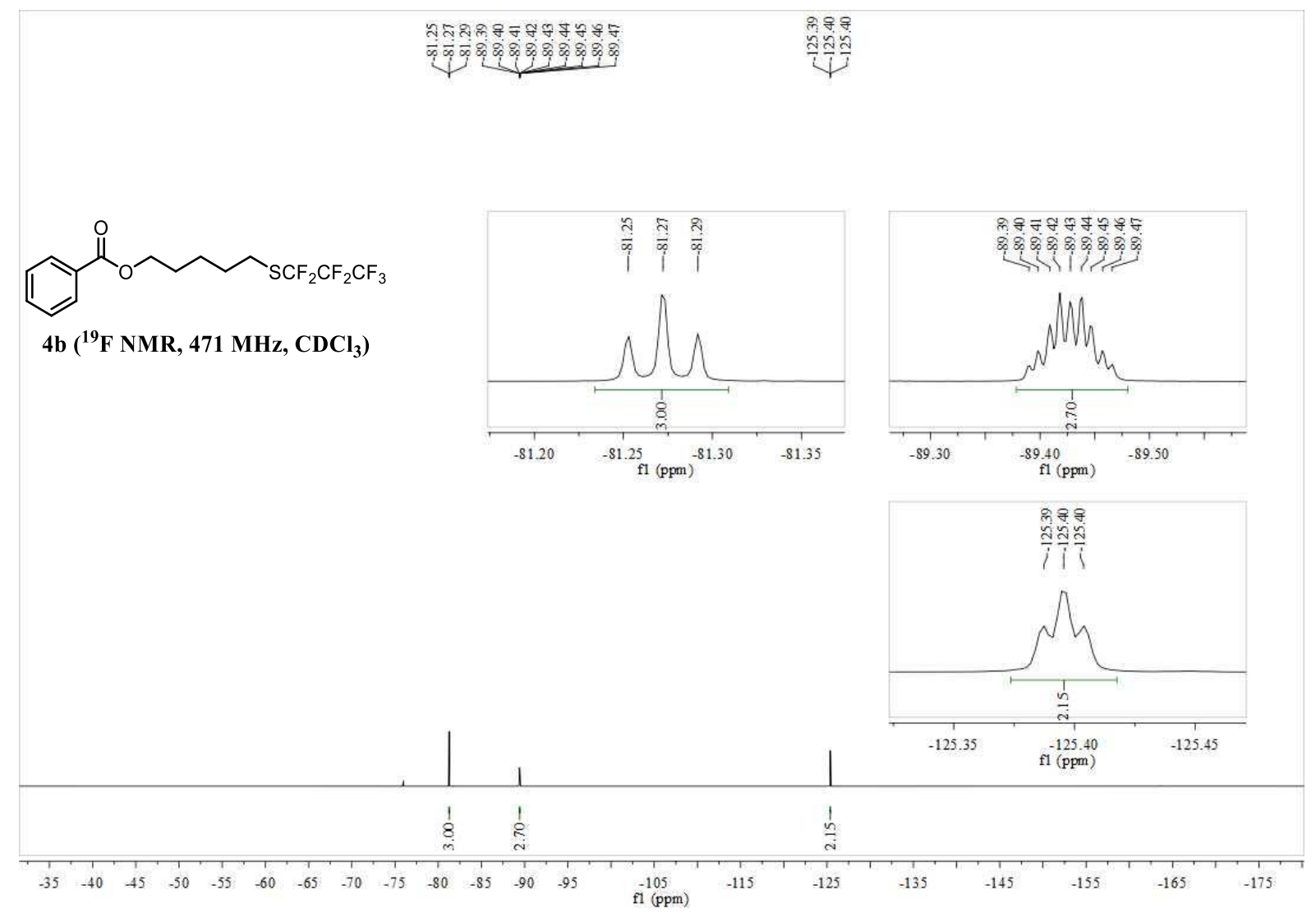

